
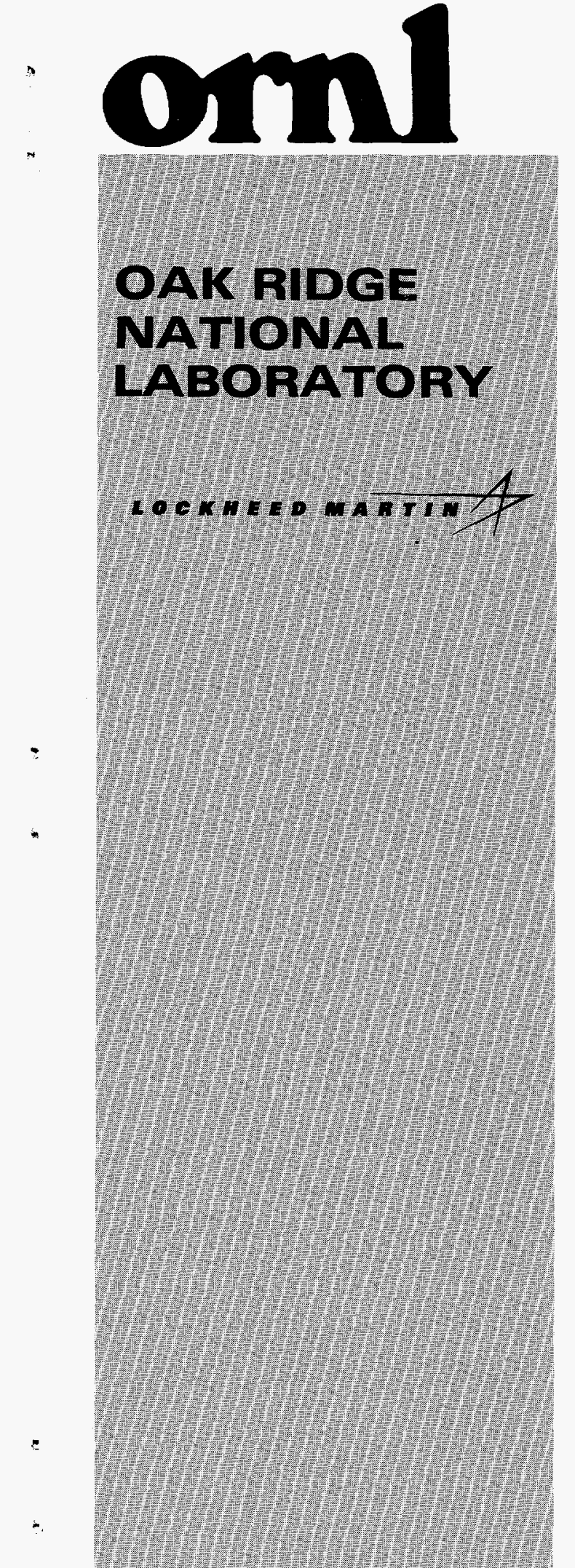

WANAGEO ANO OPERATEO BY LOCKHEED MARTIN ENEREY RESEARCH CORPORATION FOR THE UNTED STATES DEPARTIEATT OF EIERGY

\section{RECEIVED \\ OCT 141997 \\ OSTI}

ORNL/M-6094

C/ORNL93-0238

\author{
CRADA Final Report \\ for \\ CRADA Number ORNL93-0238
}

\begin{abstract}
ADVANCED QUADRUPOLE ION TRAP INSTRUMENTATION FOR LOW LEVEL VEHICLE EMISSIONS MEASUREMENTS
\end{abstract}

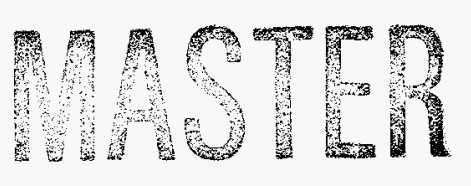

Scott A. McLuckey

Michelle V. Buchanan

Keiji G. Asano

Kevin J. Hart

Douglas E. Goeringer

Oak Ridge National Laboratory

Mark A. Dearth

Environmental Research Consortium

Ford Motor Company

Approved for Public release; distribution is unlimited. 
This report has been reproduced directly from the best available copy.

Available to DOE and DOE contractors from the Office of Scientific and Technical Information, P. O. Box 62, Oak Ridge, TN 37831; prices available from (423) 576-8401, FTS 626-8401.

Availabie to the public from the National Technical Information Service, U.S. Department of Commerce, 5285 Port Royal Road, Springfield. VA 22161.

This report was prepared as an account of work sponsored by an agency of the United States Government. Neither the United States Government nor any agency thereof, nor any of their employees, makes any warranty, express or implied, or assumes any legal liability or responsibility for the accuracy, completeness, or usefulness of any information, apparatus, product, or process disclosed, or represents that its use would not infringe privately owned rights. Reference herein to any specific commercial product, process, or service by trade name, trademark, manufacturer, or otherwise, does not necessarily constitute or imply its endorsement, recommendation, or favoring by the United States Government or any agency thereof. The views and opinions of authors expressed herein do not necessarily state or reflect those of the United States Government of any agency thereof. 


\section{DISCLAMIER}

Portions of this document may be illegible in electronic image products. Images are produced from the best available original document. 
ORNL/M-6094

C/ORNL93-0238

Chemical and Analytical Sciences Division

CRADA Final Report

for

CRADA Number ORNL93-0238

ADVANCED QUADRUPOLE ION TRAP INSTRUMENTATION
FOR LOW LEVEL VEHICLE EMISSIONS MEASUREMENTS

Scott A. McLuckey

Michelle V. Buchanan

Keiji G. Asano

Kevin J. Hart

Douglas E. Goeringer

Oak Ridge National Laboratory

Mark A. Dearth

Environmental Research Consortium

Ford Motor Company

Date Published - September 1997

Prepared for

Office of Energy Research Laboratory Technology Research Program

CRADA 93-0238

Prepared by the

OAK RIDGE NATIONAL LABORATORY

Oak Ridge, Tennessee 37831-6365

managed by

LOCKHEED MARTIN ENERGY RESEARCH CORP.

for the

U.S. DEPARTMENT OF ENERGY 


\section{TABLE OF CONTENTS}

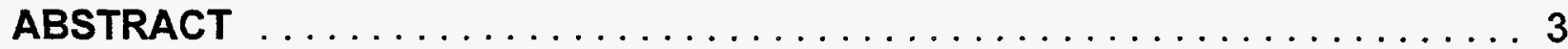

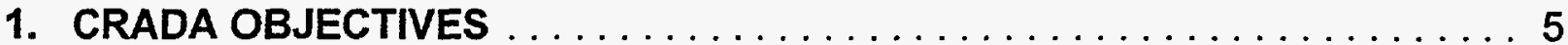

2. CRADA ACCOMPLISHMENTS AGAINST OBJECTIVES $\ldots \ldots \ldots \ldots \ldots 7$

3. CRADA BENEFITS TO THE DEPARTMENT OF ENERGY OFFICE OF ENERGY RESEARCH $\ldots \ldots \ldots \ldots \ldots \ldots \ldots \ldots \ldots$

4. TECHNICAL REPORT INTRODUCTION $\ldots \ldots \ldots \ldots \ldots \ldots \ldots \ldots \ldots \ldots$

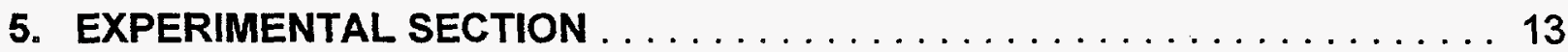

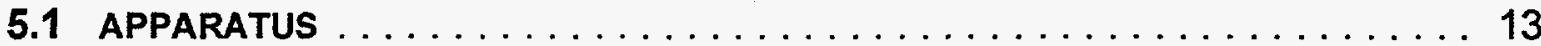

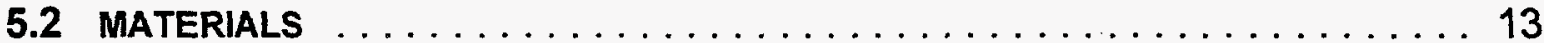

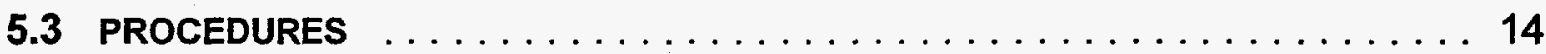

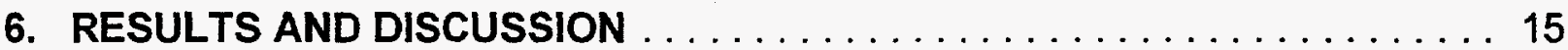

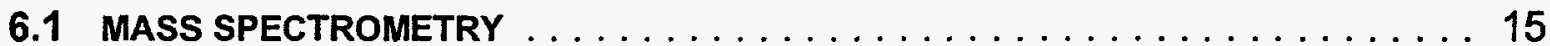

6.2 TANDEM MASS SPECTROMETRY $\ldots \ldots \ldots \ldots \ldots \ldots \ldots \ldots \ldots \ldots 20$

7. TECHNICAL REPORT CONCLUSIONS $\ldots \ldots \ldots \ldots \ldots \ldots \ldots \ldots \ldots \ldots$

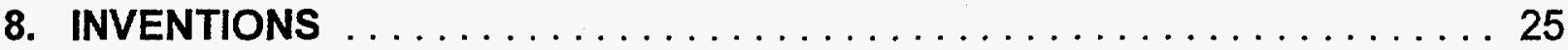

9. COMMERCIALIZATION POSSIBILITIES $\ldots \ldots \ldots \ldots \ldots \ldots \ldots \ldots \ldots 27$

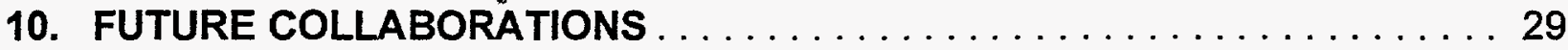

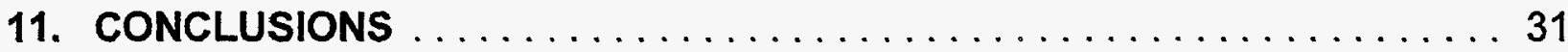

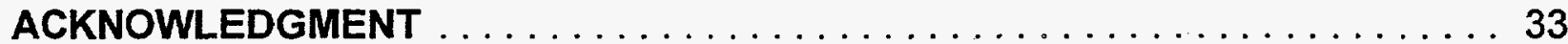

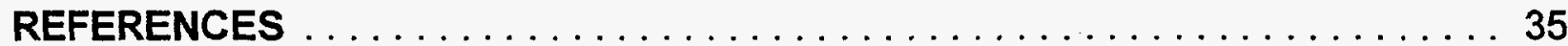

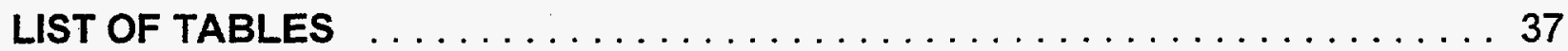

LEGENDS FOR FIGURES $\ldots \ldots \ldots \ldots \ldots \ldots \ldots \ldots \ldots \ldots \ldots \ldots \ldots \ldots$

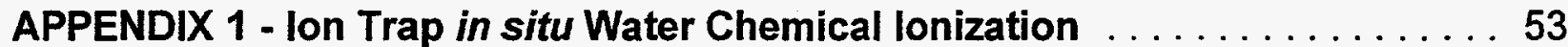

APPENDIX 2 - Software Development ...................... 59

APPENDIX 3 - Nitric Oxide Cl/ion Trap Sensitivity Data . . . . . . . . . . 63

APPENDIX 4 - Tandem Mass Spectra ..................... 73 


\title{
ADVANCED QUADRUPOLE ION TRAP INSTRUMENTATION FOR LOW LEVEL VEHICLE EMISSIONS MEASUREMENTS
}

\author{
Scott A. McLuckey \\ Michelle V. Buchanan \\ Keiji G. Asano \\ Kevin J. Hart \\ Douglas E. Goeringer \\ Oak Ridge National Laboratory \\ Oak Ridge, Tennessee 37831 \\ and \\ Mark A. Dearth \\ Environmental Research Consortium \\ Ford Motor Company \\ Dearborn, Ml 48121
}

\begin{abstract}
Quadrupole ion trap mass spectrometry has been evaluated for its potential use in vehicle emissions measurements in vehicle test facilities as an analyzer for the top 15 compounds contributing to smog generation. A variety of ionization methods were explored including ion trap in situ chemical ionization, atmospheric sampling glow discharge ionization, and nitric oxide chemical ionization in a glow discharge ionization source coupled with anion trap mass spectrometer. Emphasis was placed on the determination of hydrocarbons and oxygenated hydrocarbons at parts per million to parts per billion levels. Ion trap in situ water chemical ionization and atmospheric sampling glow discharge ionization were both shown to be amenable to the analysis of arenes, alcohols, aldehydes and, to some degree, alkenes. Atmospheric sampling glow discharge also generated molecular ions of methy-t-butyl ether (MTBE). Neither of these ionization methods, however, were found to generate diagnostic ions for the alkanes. Nitric oxide chemical ionization, on the other hand, was found to yield diagnostic ions for alkanes, alkenes, arenes, alcohols, aldehydes, and MTBE. The ability to measure a variety of hydrocarbons present at roughly 15 parts per billion at measurement rates of $3 \mathrm{~Hz}$ was demonstrated. All of the ions with potential to serve as parent ions in a tandem mass spectrometry experiment were found to yield parent-to-product conversion efficiencies greater than $75 \%$. The flexibility afforded to the ion trap by use of tailored wave-forms applied to the end-caps allows parallel monitoring schemes to be devised that provide many of the advantages of tandem mass spectrometry without major loss in measurement rate. A large loss in measurement rate would ordinarily result from the use of conventional tandem mass spectrometry experiments carried out in series for a large number of targeted components. These results have demonstrated that the ion trap has an excellent combination of sensitivity, specificity, speed, and flexibility with respect to the technical requirements of the top 15 analyzer.
\end{abstract}




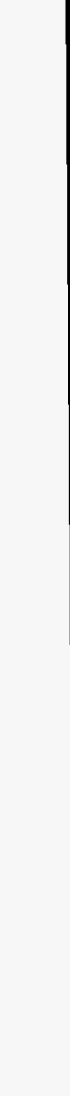




\section{CRADA OBJECTIVES}

The overall technical objective of this CRADA was to develop advanced quadrupole ion trap mass spectrometer instrumentation and protocols for the real-time quantitative analysis of trace components of engine exhausts and other vehicle emissions. The need was born of recent Federal regulations, as well as more stringent regulations enacted in California, that require auto manufacturers to market low emission vehicles (LEVs) in the near future. The Environmental Research Consortium (ERC), consisting of Chrysler, Ford, General Motors, and Navistar, has articulated needs for up to eight analytical instruments for the characterization of emissions from LEVs. Among these are a fast oxygenated hydrocarbon analyzer, a fast hydrocarbon analyzer, a direct methane and non-methane hydrocarbon analyzer, and a fast "top 15" speciation analyzer. The "top 15" refers to the most important components of engine exhaust that contribute to smog generation. The advanced ion trap research and development conducted as part of this CRADA was principally targeted at determining the extent to which an ion trap mass spectrometer can meet the criteria for the top 15 analyzer. The analytical challenge involved the development of the capability for high-speed, sensitive, and specific analysis of targeted species with the flexibility to adapt to changes in the identities of the targeted compounds. The candidate list from which the top 15 compounds are selected exceeds 150 compounds. 


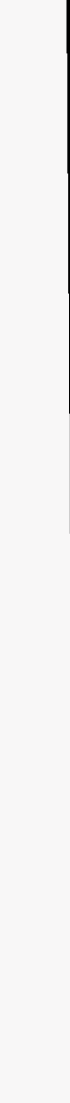




\section{CRADA ACCOMPLISHMENTS AGAINST OBJECTIVES}

The CRADA has met all of its objectives despite several obstacles encountered early in the effort. There was a delay in the project of roughly one year before the CRADA agreement was signed by all parties. The funds provided to support the ORNL effort were one-third less than the request and there were no provisions for the acquisition of capital equipment. Under such a situation, the Oak Ridge National Laboratory (ORNL) was forced to initiate the project employing existing hardware that was partly devoted to other programs. However, the ERC established a relationship with McClellan Air Force Base that solved the equipment problem. With design guidance from the ERC and ORNL, McClellan supplied two ion trap systems to the project. The first was delivered to ORNL relatively early in the project and this instrument was used to develop measurement protocols and study the ion chemistry associated with the measurements. Most of the analytical data were generated with this instrument. The second instrument was a more highly engineered prototype designed to operate in the vehicle test facility environment and was based on design criteria established by the ERC. This instrument was delivered to the ERC (at Ford) toward the end of the project.

As the project developed, the roles of the various participants were: (1) the ERC project champion coordinated all parties, established priorities and time-lines, and participated directly in hands-on mass spectrometry experiments with ORNL (this involved both Townsend discharge/triple quadrupole mass spectrometry experiments at Ford's Scientific Research Laboratory and ion trap mass spectrometry work at ORNL), (2) ORNL generated the scientific information and the analytical methodologies required for the measurement of the targeted species via ion trap mass spectrometry along with software development for providing the data in a useful format, and (3) McClellan Air Force Base provided instrumentation, engineering, and software development for the two ion trap systems.

The CRADA work demonstrated the capability for sub-second determination of multiple targeted components at low parts per billion levels with very high specificity. The nitric oxide chemical ionization approach proved to be the most universal of all ionization methods but in situ water chemical ionization and atmospheric sampling glow discharge also showed potential for at least some of the measurements of interest. The high flexibility of ion trap mass spectrometry allows for the use of all three types of ionization, although the final prototype was not configured for in situ water chemical ionization. 



\section{CRADA BENEFITS TO THE DEPARTMENT OF ENERGY OFFICE OF ENERGY RESEARCH}

The principle benefit of this work to the Department of Energy Office of Energy Research is in the expanded knowledge base it provided to the mass spectrometry researchers at ORNL. Specifically, exposure to the problems associated with vehicle emissions measurements of low level hydrocarbons and oxygenated hydrocarbons required building strength in the gas-phase ion chemistry of ions derived from such species. It has highlighted areas where fundamental information is lacking thereby suggesting important research directions. The knowledge gained in this work served as the basis for a successful proposal to the Office of Basic Energy Sciences on the topic of hydrocarbon and oxygenated hydrocarbon ion chemistry. Furthermore, many of the compounds present in engine exhaust have significance beyond their introduction to the environment via automobile emissions. For example, isoprene (2-methyl-1,3-butadiene) is a major reactant in smog production and is primarily produced by vegetation. It is one of the major compounds of interest in tropospheric chemistry for the development of models to understand urban air pollution. This work, therefore, has generated interest in the atmospheric chemistry community and has led to the discussions of collaboration between ORNL researchers and atmospheric chemists in academia and at NOAA. 


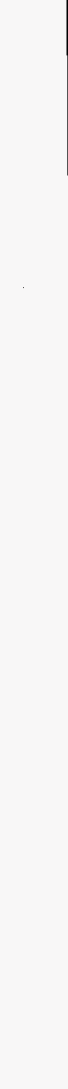




\section{TECHNICAL REPORT INTRODUCTION}

Increasing emphasis on the reduction of emissions of smog producing species from automobiles has recently resulted in more stringent federal, and in some cases, state emission requirements. In response, the automotive industry is developing new engines that produce significantly lower levels of smog precursors, such as hydrocarbons and oxygenated hydrocarbons. ${ }^{1}$ Enabling technologies are required to support the development of low emission vehicles including analytical technologies and methodologies for rapid, sensitive, and specific monitoring of engine exhaust components. There is a need for analytical methodologies capable of detecting a range of targeted species in the sub-parts-per-million concentration range with good specificity and with time resolution on the order of 1 second or less. Methodologies currently in use tend to rely heavily on chromatographic techniques and do not meet all of the requirements for application to fast dynamic exhaust gas measurements for multiple components. ${ }^{2}$

Several approaches based on mass spectrometry show promise for rapid determination of multiple low level exhaust gas components. These include, for example, resonance enhanced multiphoton ionization/time-of-flight mass spectrometry ${ }^{3}$ and low energy charge exchange mass spectrometry, ${ }^{4}$ including nitric oxide chemical ionization. Nitric oxide has a long history as a reagent gas for analytical mass spectrometry ${ }^{6-14}$ and has been applied to hydrocarbon analysis using a Townsend discharge ${ }^{15}$ and to engine exhaust using atmospheric pressure chemical ionization (APCl). ${ }^{16}$ The latter study showed that $\mathrm{APCl}$ using nitric oxide as a reagent gas improved sensitivity and reduced matrix effects (primarily arising from water) relative to the use of APCI without addition of NO. Triple quadrupole tandem mass spectrometry data were also reported for the major parent ions derived from a variety of engine exhaust components of interest.

The combination of selective chemical ionization, such as that afforded by the nitrosonium ion, $\mathrm{NO}^{+}$, with tandem mass spectrometry has the potential to meet the analytical requirements for a fast, highly specific, and sensitive means for the analysis of low level organic species in engine exhaust. The low relative cost and size of the quadrupole ion trap, its amenability to field applications, ${ }^{17,18}$ and the high degree of experimental flexibility characteristic of ion trapping instruments make it attractive for consideration as the tandem mass spectrometer for use in vehicle test facilities. Furthermore, the ion trap is particularly amenable to coupling with relatively high pressure ionization sources. ${ }^{19}$ For example, atmospheric sampling glow discharge for the ionization of trace organic species in air ${ }^{20}$ has been coupled with a quadrupole ion trap for the detection of trace concentrations of organic high explosives. ${ }^{21}$ A similar approach, modified for the selective ionization of hydrocarbons and oxygenated hydrocarbons, holds promise for engine exhaust measurements. This technical report emphasizes mass spectrometry and tandem mass spectrometry results obtained from combining nitric oxide chemical ionization in a glow discharge ion source with quadrupole ion trap mass spectrometry with emphasis on the measurement of hydrocarbons at low parts-per-million to low parts-perbillions concentration levels. Expanded descriptions of in situ chemical ionization studies, software development, sensitivity data, and tandem mass spectra are given as appendices. 


\section{EXPERIMENTAL SECTION}

\subsection{APPARATUS}

Figure 1 shows a side-view schematic of an atmospheric sampling glow discharge ionization source mated with a Teledyne-Hitachi $3 D Q$ quadrupole ion trap system (Teledyne Electronic Technologies, Mountain View, CA). Modifications to the commercially available $3 D Q$ instrument involved removal of the filament assembly from the front flange of the $3 D Q$ vacuum system and attachment of the glow discharge source, which was designed to replace the filament assembly. A lens stack was also added to focus ions that exit the glow discharge source onto the hole in the center of the end-cap closest to the filament assembly. This lens stack is supported by the ion source flange and the existing feed-throughs were used to apply the necessary potentials to the lenses. Ions were formed by pulsing the glow discharge such that the pulse duration defined the ion accumulation period of the ion trap experiment. Operating in a pulsed mode has two advantages: (1) no ion gating is required as it is with continuous ion sources, and (2) background noise arising from the discharge during mass analysis is eliminated. ${ }^{21,22} \mathrm{~A}$ pulse supplied by the $3 \mathrm{DQ}$ electronics is used to trigger a variable length pulse generator (Model 8010, Berkeley Nucleonics, Berkeley, CA) which gates a high voltage solid state pulser (Model GRX-1.5K-E, Directed Energy Inc., Fort Collins, CO). The pulser serves as a fast switch between a potential of roughly $-400 \mathrm{~V}$, which is applied to the sampling capillary to strike and maintain the discharge, and ground. The voltage and current necessary to strike and maintain the discharge is provided by an ORTEC (Oak Ridge, TN) Model 556 power supply. Two half-plates are also mounted within the ion source volume. For these studies, one was grounded and a relatively small positive potential, as supplied by an ORTEC Model 556 power supply, was applied to the other to optimize positive ion signals and $\mathrm{NO}^{+}$attachment to the aromatic hydrocarbons. Table 1 summarizes typical operating conditions used for ion formation, accumulation, and detection.

Data were also collected using in situ ion trap chemical ionization ${ }^{23}$ with the hydronium ion serving as the reagent ion and using atmospheric sampling glow discharge ionization without the addition of nitric oxide to test the applicability of other ionization approaches to various species of interest. In the former case, a Finnigan lon Trap Mass Spectrometer (Finnigan, San Jose, CA) was used to coliect data taking advantage of the ubiquitous water in the vacuum system to serve as the reagent gas. The glow discharge data were collected under conditions essentially identical to those listed in Table 1 except that no flow of NO-doped nitrogen was added to the ion source.

\subsection{MATERIALS}

Mixture analysis studies and analytical curves were determined using a gas mixture standard supplied by Scott Specialty Gases (Plumsteadville, PA). This cylinder contained twenty low level components in nitrogen ranging in concentration from $2.5 \mathrm{ppm}$ to $0.6 \mathrm{ppm}$ (see Table 2). Identification of parent ions formed via nitric oxide chemical ionization from 
individual mixture components were determined by introducing each component individually into the ion source. These compounds were obtained from Chem Service, Inc. (West Chester, PA). Studies with formaldehyde and acetaldehyde were performed using gas standards of $5 \mathrm{ppm}$ and $1 \mathrm{ppm}$, respectively, in nitrogen provided by Scott Specialty Gases. Dilutions of the gas standards were made with zero grade air (Air Products, Allentown, PA).

\subsection{PROCEDURES}

The NO/N $\mathrm{N}_{2}$ mix was admitted into the ion source via a Granville-Philips variable leak valve through an inlet adapted to enter through the source pump-out port. The sample gas was admitted via a Granville-Philips variable leak valve leading to the sampling capillary tube. The flow rate through this tube was maintained at $15 \mathrm{ml} / \mathrm{min}$. The standard gas mix flow was maintained at $15 \mathrm{ml} / \mathrm{min}$ and, for sensitivity studies, was diluted prior to the valve leading to the sampling capillary tube by adding measured flows of zero air. Gas flows in excess of the sampling rate of the ion source were exhausted to the laboratory. Mass flow controllers (Matheson, Inc., East Rutherford, NJ) were used to establish the gas flows and mixing took place in stainless steel and Teflon tubing prior to the valve leading to the sampling capillary. For the glow discharge mass spectra and MS/MS spectra shown in Figs. 2, 3, 6, and 7, an ion injection period of $50 \mathrm{~ms}$ was used. The sensitivity studies were performed using an ion accumulation period of $100 \mathrm{~ms}$. Mass analysis was typically performed over a mass-to-charge range of $20-200$ and at a rate of $10^{4} \mathrm{Da} / \mathrm{s}$ to yield a total mass scan time of about $18 \mathrm{~ms}$. 


\section{RESULTS AND DISCUSSION}

\subsection{MASS SPECTROMETRY}

In developing low emission vehicles, there is a need for an analytical tool to monitor rapidly roughly 10 to 20 targeted species in a vehicle test facility environment. The targeted species are those deemed to be most important for smog generation and the identities of these components may vary depending upon combustion conditions, fuel composition, etc. The candidate list from which the highest priority targeted compounds are drawn exceeds one hundred compounds. This situation highlights the need for flexibility and adaptability of the analytical methodology used in vehicle test testing. To illustrate much of what has been learned regarding the use of the ion trap for such an application, emphasis is placed on a twenty component standard gas mixture in nitrogen commonly used in the automotive industry for emissions testing. Identities of the components and their concentrations in the gas cylinder are listed in Table 2. Also listed are the respective ionization energies, proton affinities, and estimated hydride ion affinities for all of the mixture components as well as for water and nitric oxide. The thermochemical data were obtained from published sources ${ }^{24,25}$ and the hydride ion affinities (HIAs) were estimated by the relation:

$$
H I A\left(R^{+}\right)=\Delta H_{f}\left(H^{-}\right)+\Delta H_{f}\left(R^{+}\right)-\Delta H_{f}(R H)
$$

The HIA values in some instances are rough estimates due to uncertainty in the structures of the cations formed by hydride abstraction. In some cases, a range of values are listed which represent the known range in $\Delta \mathrm{H}_{f}\left(R^{+}\right)$for possible $R^{+}$structures.

Figure 2 compares ion trap mass spectra obtained from the gas mix standard using three methods for ionization. Figure $2 a$ shows the spectrum obtained via in situ chemical ionization using $\mathrm{H}_{3} \mathrm{O}^{+}$as the reagent ion. Proton transfer from the hydronium ion to an analyte molecule is exothermic provided the proton affinity of the analyte is greater than that of water (see Table 2). Abundant signals for the protonated arenes (benzene, toluene, and the $\mathrm{C}_{2}$ - and $\mathrm{C}_{3}$-benzenes) are apparent. Signals are also observed over the mass-tocharge regions of $67-73,53-57$, and $40-46$, many of which are likely to arise from hydrocarbons. Some of these ions can arise from protonation of the alkenes since proton transfer is exothermic for most of them. For example, ions at $\mathrm{m} / \mathrm{z} 71$ could arise from protonation of cis-2-pentene and trimethylethylene. However, the genealogy of the ions is not clear since they also might arise, at least in part, from fragmentation of alkanes. No signals exclusively attributable to protonation of the alkanes of the mixture are apparent. For example, no signals are observed corresponding to the protonated molecules. The proton affinities for the alkanes in the gas mix standard have not been reported so it is possible that proton transfer from the hydronium ion to the alkanes does not occur to an appreciable extent. The proton affinity of ethane, for example, is reported to be 142.5 $\mathrm{kcal} / \mathrm{mol}^{24}$ and would therefore not be expected to extract a proton from the hydronium ion. However, it is also possible that proton transfer followed by fragmentation to yield ions at 
lower mass-to-charge ratios takes place. In any case, it is clear that in situ water chemical ionization is effective for the arenes and, perhaps the alkenes, but does not yield diagnostic ions for the alkanes. Protonation of the alkenes might also give rise to a significant degree of fragmentation but no conclusions can be drawn regarding this possibility from Fig. 2a. It is also noteworthy that no protonated methyl-t-butyl ether (MTBE) is apparent in the spectrum. The proton transfer reaction is exothermic by roughly $35 \mathrm{kcal} / \mathrm{mol}$ (see Table 2). It seems likely, therefore, that proton transfer, if it occurs, is followed by fragmentation (probably via methanol loss to yield the butyl cation). An expanded description of ion trap in situ chemical ionization studies is included in Appendix 1.

Figure $2 \mathrm{~b}$ shows the ion trap mass spectrum of the gas mixture using atmospheric sampling glow discharge without the addition of nitric oxide. This spectrum is almost identical to that of Fig. $2 a$ in terms of mass-to-charge values of the major ions. This is not a surprising observation because it has been noted previously that under the mild glow discharge conditions used here proton transfer from proton hydrates is the major ionization mechanism for species with proton affinities greater than water.$^{20} \mathrm{~A}$ significant difference between water chemical ionization within the ion trap and ionization in the glow discharge source is that other ionization mechanisms, such as charge transfer, can occur in the discharge since there is a greater variety of ions available for reaction in the high pressure source. This may account for the observation of an intense signal corresponding to the molecular ion of MTBE at $\mathrm{m} / \mathrm{z} 88$. Tandem mass spectrometry of this ion results almost exclusively in the formation of a product ion at $\mathrm{m} / \mathrm{z} 57$, which is consistent with the loss of a methoxy radical to yield the t-butyl cation. However, signals corresponding to molecular ions of the alkanes are very small, at best, indicating that normal atmospheric sampling glow discharge, while effective for MTBE, the arenes, and possibly some of the alkenes, is ineffective in yielding characteristic ions from room temperature alkanes.

When $0.3-0.4$ torr of $1-10 \%$ NO in nitrogen is admitted into the glow discharge source (total ion source pressure of 0.8-1.4 torr), by far the base peak in the positive ion mass spectrum arises from $\mathrm{NO}^{+}$. When lab air accounts for the balance of the source pressure small signals ( $<5 \%$ relative abundance) are observed that correspond to $\left(\mathrm{H}_{2} \mathrm{O}\right)_{2} \mathrm{H}^{+}$ and $\left(\mathrm{H}_{2} \mathrm{O}\right) \mathrm{NO}^{+}$. Figure $2 \mathrm{c}$ shows the mass spectrum of the gas standard mixture obtained under conditions that optimize signals arising from attachment of $\mathrm{NO}^{+}$to the arenes and dienes (see below). This spectrum contrasts sharply with those of Figs. $2 a$ and $2 \mathrm{~b}$. Figure $2 c$ indicates that all three mechanisms noted previously for nitric oxide chemical ionization of hydrocarbons ${ }^{14-16}$ give rise to products in the mass spectrum, viz.:

$$
\begin{array}{ll}
\mathrm{M}+\mathrm{NO}^{+} \ldots(\mathrm{M}-\mathrm{H})^{+}+\mathrm{NOH} & \text { hydride abstraction } \\
\mathrm{M}+\mathrm{NO}^{+} \ldots \mathrm{M}^{+}+\mathrm{NO} & \text { electron transfer } \\
\mathrm{M}+\mathrm{NO}^{+} \ldots(\mathrm{M}+\mathrm{NO})^{+} & \mathrm{NO}^{+} \text {attachment }
\end{array}
$$


The compounds giving rise to each peak in the spectrum, as indicated by the labels in the figure, were determined by admitting each compound present in the gas standard (mixed with air) separately into the ion source. For example, under the conditions used to obtain Fig. $2 \mathrm{c}$, the arenes and dienes react almost exclusively via $\mathrm{NO}^{+}$attachment. Butene and the pentenes show both $\mathrm{NO}^{+}$attachment and electron transfer. However, the relative contribution of electron transfer is greater with the pentenes than with 1-butene and the pentenes also show a significant contribution from hydride ion abstraction. The alkanes, on the other hand react exclusively via hydride abstraction. MTBE was found to form the molecular ion and the t-butyl cation under these ionization conditions. Examination of Table 2 provides some insight into why the ionization products arising from water $\mathrm{Cl}$ and nitric oxide $\mathrm{Cl}$ are so different. The ionization energies of the alkanes, for example, are too high to be ionized by electron transfer to $\mathrm{NO}^{+}$but hydride abstraction is exothermic for all of the alkanes. Hydride abstraction does not occur with water $\mathrm{Cl}$. The alkenes and the arenes can be ionized either by electron transfer or $\mathrm{NO}^{+}$attachment. As indicated below in the MS/MS studies, the $\left(\mathrm{M}+\mathrm{NO}^{+}\right)$adduct is, in fact, an intermediate in the electron transfer reaction. Of all of the low level components in the mixture, only ethene and propene show no clearly obvious ions in the mass spectrum. Neither are expected to react via electron transfer or hydride abstraction. No $\mathrm{NO}^{+}$adducts are observed either. However, it is possible that any such ions could be dissociated as a result of the trapping process (see below) yielding $\mathrm{NO}^{+}$since the ionization energies of ethene and propene exceed that of NO. In any case, although the relative ion signal arising from the molecular ion of MTBE is somewhat lower with nitric oxide $\mathrm{Cl}$ than with atmospheric sampling glow discharge, nitric oxide $\mathrm{Cl}$ is clearly an effective means for ionizing almost all of the components of the mixture, including the alkanes.

The data of Fig. $2 c$ were collected using a low mass-to-charge cutoff of 9 . The low mass-to-charge exclusion limit is an important parameter in the capture of ions injected from external ion sources ${ }^{26,27}$ in that it affects the efficiency with which ions are trapped and can influence the degree to which ions fragment upon capture. ${ }^{28}$ Fragmentation upon ion injection into the ion trap has been attributed to inelastic ion/helium collisions during the process of removal of ion kinetic energy of captured ions. ${ }^{19}$ The relative abundances of many of the ions formed via nitric oxide $\mathrm{Cl}$ were found to be highly sensitive to the low mass-to-charge cut-off during the ion accumulation period. Figure 3, for example, shows the glow discharge nitric oxide $\mathrm{Cl}$ mass spectrum acquired under conditions identical to those of Fig. $2 \mathrm{c}$ except that the low mass-to-charge cutoff during ion accumulation was $\mathrm{m} / \mathrm{z}$ 24 rather than $\mathrm{m} / \mathrm{z} 9$. Low $\mathrm{m} / \mathrm{z}$ peaks, such as those at $\mathrm{m} / \mathrm{z} 57,43$, and 41 are increased relative to the higher $\mathrm{m} / \mathrm{z}$ peaks, which suggests an increase in the degree of fragmentation. A clear indication of fragmentation of the $\left(\mathrm{M}+\mathrm{NO}^{+}\right)$ions is the diminution of the signals arising from the $\left(\mathrm{M}+\mathrm{NO}^{+}\right)$ions and the appearance of $\mathrm{M}^{+}$ions. This is most clearly evident with the arenes whereby decreases in the ion signals from $\left(\mathrm{M}+\mathrm{NO}^{+}\right)$at $\mathrm{m} / \mathrm{z}$ 108 (benzene), 122 (toluene), 136 (the $C_{2}$-benzenes), and 150 (the $C_{3}$-benzenes) are met with the appearance of $\mathrm{M}^{+}$ions at $\mathrm{m} / \mathrm{z} 78$ (benzene), 92 (toluene), 106 (the $\mathrm{C}_{2}$-benzenes), and 120 (the $\mathrm{C}_{3}$-benzenes). This observation indicates that there is an optimum low massto-charge cutoff level that balances absolute signal with an acceptable degree of fragmentation. The optimum level for these ions is roughly $\mathrm{m} / \mathrm{z} 9$. This level also 
determines the frequency dispersion of the ions during the ion accumulation process. As the low mass-to-charge cut-off used during ion accumulation decreases, the frequency dispersion of the ions also decreases, which can complicate mass-selective ion accumulation, ${ }^{30}$ particularly for the high $\mathrm{m} / \mathrm{z}$ ions. While it proved to be possible to effect mass-selective ion accumulation using a low mass-to-charge cut-off of $\mathrm{m} / \mathrm{z} 9$, the effective mass selection resolution obtainable without ejecting a measurable fraction of parent ions was roughly 50 . Using a mass-selection period after ion accumulation with a higher low mass-to-charge cutoff to provide greater parent ion frequency dispersion allowed for mass selection resolution of several hundred. However, the latter approach does not prevent ion/ion interactions from affecting dynamic range. ${ }^{29}$

While this report emphasizes the application of nitric oxide chemical ionization to a mixture of hydrocarbons, the applicability to several oxygenated hydrocarbons of interest was also evaluated. Specifically, nitric oxide chemical ionization was applied to acetaldehyde, methanol, ethanol, and formaldehyde. In the case of the methanol, the major ion observed corresponded to $\left(\mathrm{M}+\mathrm{NO}^{+}\right)$species, in analogy with nitric oxide $\mathrm{APCl}$ data previously reported. ${ }^{16}$ Ethanol and acetaldehyde reacted primarily by hydride abstraction. Formaldehyde (5 ppm in nitrogen) yielded an intense ion at $\mathrm{m} / \mathrm{z} 90$, rather than the expected $\left(\mathrm{M}+\mathrm{NO}^{+}\right)$at $\mathrm{m} / \mathrm{z} 60$. Collisional activation of the ion at $\mathrm{m} / \mathrm{z} 90$ yielded primarily a product ion at $\mathrm{m} / \mathrm{z} 30$ (either $\mathrm{NO}^{+}$or the formaldehyde molecular ion) and a small signal at $\mathrm{m} / \mathrm{z}$ 61. These observations are interpreted as evidence for paraformaldehyde formation, primarily $\left(\mathrm{CH}_{2} \mathrm{O}\right)_{2}$, with $\mathrm{NO}^{+}$attachment to the dimer (although the formation of $\left[\mathrm{CH}_{2} \mathrm{O}_{3}{ }^{+}\right.$cannot be precluded). The $\mathrm{m} / \mathrm{z} 61$ product ion is interpreted as arising from $\mathrm{HCO}$ loss from the $\left(\mathrm{CH}_{2} \mathrm{O}\right)_{2} \mathrm{NO}^{+}$ion.

In general, the time resolution for real-time monitoring applications tends to be inversely related to the concentration of the targeted compounds, at least at concentration levels that require signal averaging. In the ion trap experiment the duty cycle of the measurement, defined as the ion accumulation period divided by the total time to execute the complete cycle of events that constitutes an ion trap scan, is variable. The time required to mass analyze the ions (typically $18 \mathrm{~ms}$ in these studies) is fixed by the product of the scan rate and scan range. The ion accumulation time, however, can be varied from less than one millisecond to hundreds of milliseconds. The duty cycle can therefore range from less than one percent using short ion accumulation times to greater than ninety percent using long ion accumulation times. However, greater duty cycles result in $\mathrm{M}^{+}$of lower measurement rates. Based on the finite scan rate of the ion trap, the highest realistic measurement rate is roughly $33 \mathrm{~Hz}$, assuming an ion accumulation time of $10 \mathrm{~ms}$. It was found that the components of the undiluted gas mix standard (roughly $1 \mathrm{ppm}$ concentrations, see Table 2) could be monitored using a $10 \mathrm{~ms}$ ion accumulation period without averaging scans. However, at significantly lower concentrations, either longer ion accumulation times (greater duty cycles) or averaging, or both, were required. As a result of the limited ion storage capacity of the ion trap, ${ }^{30}$ the ion accumulation time also determines the concentration range over which signals are linear with concentration. In the absence of automated means for varying ionization time in response to signal levels, ${ }^{32}$ a capability available on some commercial ion trap systems but not with the instrument 
used in these studies, an ion accumulation period must be chosen as a best compromise between concentration sensitivity, measurement rate, and linearity over a concentration range of interest. Data are reported here using a $100 \mathrm{~ms}$ ion accumulation period, which was found to yield performance characteristics likely to be desirable in many vehicle test testing scenarios. However, it should be noted that the ion accumulation period is readily variable so that either longer or shorter accumulation times could be selected to optimize performance over other ranges of concentration than shown here.

Figure 4 compares a mass spectrum acquired from the undiluted gas mixture (Fig. 4a) using a $100 \mathrm{~ms}$ ion accumulation period with a mass spectrum acquired with a 67 -fold dilution (Fig. 4b) under otherwise identical conditions. Figure 4a represents a spectrum acquired for analyte species at roughly the $1 \mathrm{ppm}$ level (see Table 2) whereas Fig. $4 \mathrm{~b}$ represents a spectrum acquired at roughly the $15 \mathrm{ppb}$ level. Each spectrum is the average of three scans consisting of the ion accumulation period and mass scan yielding a total individual scan time of about $120 \mathrm{~ms}$. Acquiring data under these conditions, therefore, allows for a measurement repetition rate of about $3 \mathrm{~Hz}$. There are several noteworthy observations that can be made in the comparison of Fig. 4. For example, essentially all of the components that are apparent in the $1 \mathrm{ppm}$ spectrum are also evident in the $15 \mathrm{ppb}$ spectrum and the relative abundances of the peaks appear not to change dramatically, at least within the scan-to-scan reproducibility of the data. However, the peaks tend to be sharper in the $15 \mathrm{ppb}$ spectrum, particularly at lower mass-to-charge. This is due to reduced contribution from ion/ion interactions relative to the data acquired with the $1 \mathrm{ppm}$ sample. It is also noteworthy that despite a nearly 70 -fold reduction in concentration there is only a roughly six-fold loss in peak heights in the two spectra. The latter two observations are related in that the signal height versus concentration response is not linear over this concentration range when a fixed ion accumulation time of $100 \mathrm{~ms}$ is used. Appendix 2 provides a description of the software developed to collect sensitivity data.

Figure 5 shows the peak area versus concentration curves for the ions at $\mathrm{m} / \mathrm{z} 70$, $\mathrm{m} / \mathrm{z} 71$, and $\mathrm{m} / \mathrm{z} 108$ obtained using conditions very similar to those used to acquire the data of Fig. 4. For the sake of visual clarity, only these three ions are plotted and they were selected because they represent the three major ion types; viz., $\mathrm{M}^{+}$(pentenes), ( $\mathrm{M}$ $\mathrm{H})^{+}\left(\right.$isopentane), and $\left(\mathrm{M}+\mathrm{NO}^{+}\right)$(benzene). (Appendix 3 provides a more comprehensive summary of sensitivity data.) Signal responses are linear with concentration for all three ions from $15 \mathrm{ppb}$ to several hundred ppb and begin to level off thereafter. This non-linearity does not arise from the ionization process because glow discharge ionization has been shown to be linear over as many as six orders of magnitude. ${ }^{20}$ Rather, the non-linearity results from the finite ion storage capacity of the ion trap. Two tactics can be used to improve the linear dynamic range. One involves the use of mass-selective ion accumulation so that the full ion storage capacity of the ion trap can be available for a limited number of targeted ions, at the expense of monitoring all ions simultaneously. The other tactic is to employ data dependent ion accumulation periods, as mentioned above, to alter the sensitivity of the ion trap in a concentration dependent fashion. 


\subsection{TANDEM MASS SPECTROMETRY}

Tandem mass spectrometry is widely known for the added specificity that it can provide over single stage mass spectrometry and for the reduction in chemical noise associated with complex mixture analysis. ${ }^{33,34}$ Quadrupole ion trap tandem mass spectrometry is recognized for the high efficiency it can provide, ${ }^{35,36}$ in terms of converting parent ions into detectable product ions. In favorable cases, efficiencies can approach $100 \%$ thereby providing the advantage of reducing chemical noise without sacrificing analyte signal. Under such circumstances, tandem mass spectrometry can provide analytical performance for a targeted compound in a mixture analysis scenario that is comparable to analysis of the pure compound, provided there are no matrix effects in the ionization process. All of the candidate parent ions for the components in the gas standard mixture larger than propene yield high efficiencies $(>75 \%)$. An example is provided in Fig. 6 which shows that the parent ions arising from electron transfer to the pentenes (both cis2-pentene and trimethylethylene) $(\mathrm{m} / \mathrm{z} 70)$ fragment predominantly to products at $\mathrm{m} / \mathrm{z} 55$ (methyl loss), $\mathrm{m} / \mathrm{z} 42$ (ethylene loss), and $\mathrm{m} / \mathrm{z} 41$ (ethyl loss). The signals arising from these product ions account for slightly over $100 \%$ of the signal of the isolated parent ion. The apparent greater than $100 \%$ efficiency is attributed to fluctuations in parent ion signal of up to $20 \%$ between the time Figs. $6 \mathrm{a}$ and $6 \mathrm{~b}$ were acquired. Table 3 lists the parent ions and major product ions relevant to the various components in the gas standard mixture. In cases where several product ions are listed, the most abundant product ion is underlined. (Appendix 4 provides tandem mass spectra from which much of Table 3 was constructed.) In the case of the $(\mathrm{M}+\mathrm{NO})^{+}$ions, the molecular ion is the only product ion obtained under ion trap collisional activation conditions. These ions are formed with essentially $100 \%$ efficiency. If specificity greater than that provided by the $(M+N O)^{+} / M^{+}$. parent/product pair is required, such as the distinction between ethylbenzene and o-xylene, the ion trap is capable of performing $\mathrm{MS}^{3}$ whereby the molecular ion is interrogated further. Such data have been recorded but are not shown here.

While the efficiency of the ion trap tandem mass spectrometry experiment can be $100 \%$, there is a price to pay in terms of duty cycle. For a single targeted compound, the change in duty cycle in going from MS to MS/MS is determined by the additional time required for the second stage of mass spectrometry. The additional time associated with the MS/MS experiment includes the ion activation period (typically $10-20 \mathrm{~ms}$ ) and any time associated with ion isolation. Ion isolation procedures, however, need not adversely affect duty cycle if ion isolation is performed during the ion accumulation process. Mass selective ion accumulation in the quadrupole ion trap using tailored wave-forms applied to the endcap electrodes, for example, has been demonstrated for ions injected from external ion sources. ${ }^{29,30,37}$ The ion activation period is, therefore, the major source of additional time in the scan. When ion accumulation periods on the order of $100 \mathrm{~ms}$ are used, as is typical with the present application, only a modest loss in duty cycle (less than 10\%) is associated with going from MS to MS/MS for a single targeted compound. For example, essentially identical analytical curves were obtained for benzene by monitoring the $\left(\mathrm{M}+\mathrm{NO}^{+}\right)$signal in MS mode (see above) and by monitoring the molecular ion of benzene following isolation and collision activation of the $\left(\mathrm{M}+\mathrm{NO}^{+}\right)$ion (data not shown). However, there is a much 
more significant loss in duty cycle when multiple targeted compounds are of interest and when a single scan procedure is employed for each targeted compound of interest. As a consequence, the sequential nature of individual MS/MS experiments adversely affects the time resolution with which measurements can be made for any given mixture component. The loss in duty cycle and time resolution is inversely related to the number of targeted components to be monitored.

While with conventional beam-type tandem mass spectrometry there is little recourse for the loss of duty cycle and time resolution associated with monitoring in series a number of targeted species, ion trapping instruments offer a means for ameliorating this problem. Via the use of tailored wave-forms for parent ion selection and collisional activation, it is possible to subject multiple arbitrarily selected parent ions to collisional activation within a single ion trap scan. This procedure has recently been described and illustrated within the context of a trace explosives detection scenario ${ }^{21,38}$ and is referred to as a parallel monitoring procedure because it allows for several targeted species to be monitored simultaneously. The procedure trades some of the specificity afforded by MS/MS for speed. A loss in parent ion/product ion genealogical information results from collecting simultaneously a number of parent ions of interest and simultaneously activating all parents. However, there is a gain in speed and duty cycle over sequential MS/MS experiments in direct proportion with the number of parent ions collected simultaneously.

The engine exhaust application is one that can benefit from the parallel monitoring procedure because the mixture of species presented to the ion source is of limited complexity. That is, the mixture of ions arising from nitric oxide chemical ionization of engine exhaust is largely limited to hydrocarbons and oxygenated hydrocarbons. The use of a parallel monitoring procedure for the engine exhaust scenario is illustrated here for the dienes and arenes present in the gas mix standard. Figure 7a shows a spectrum of parent ions in which a filtered-noise field ${ }^{39}(F N F)$, the form of tailored wave-form supplied with the Teledyne 3DQ instrument, was employed to isolate parent ions at $\mathrm{m} / \mathrm{z}$ values 150,136 , $122,108,98$, and 84 corresponding to the $\left(\mathrm{M}+\mathrm{NO}^{+}\right)$ions of the $\mathrm{C}_{3}$-benzenes, $\mathrm{C}_{2}$-benzenes, toluene, benzene, 2-methyl-1,3-butadiene, and 1,3-butadiene, respectively. Figure $7 \mathrm{~b}$ shows the spectrum resulting from the simultaneous activation of the six selected parent ions, using an FNF designed to resonantly excite simultaneously each of the parent ions. The expected product ions, the molecular ions of each component, along with a moderate $\mathrm{NO}^{+}$signal are clearly observed illustrating that monitoring the $\mathrm{m} / \mathrm{z}$ values corresponding to the molecular ions of the targeted compounds can provide for their rapid determination with significantly enhanced specificity over single stage mass spectrometry alone. Interpretation of the data arising from this experiment is straightforward in that none of the product ions formed are isobaric with a parent ion and none of the product ions are shared by more than one parent ion. 


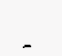




\section{TECHNICAL REPORT CONCLUSIONS}

Nitric oxide chemical ionization in a glow discharge ionization source coupled with a quadrupole ion trap allows for the determination of a range of hydrocarbons that includes alkanes, alkenes, and arenes. The capability for measurements at low parts per billion levels at roughly $3 \mathrm{~Hz}$ has been demonstrated. The linear dynamic range for the measurements is ultimately limited by the finite ion storage capacity of the ion trap. In the absence of mass-selective ion accumulation techniques or data dependent control of ionization time, linear dynamic range is limited to one to two orders of magnitude when a $100 \mathrm{~ms}$ ion accumulation time is employed and when roughly 20 ions of differing mass-tocharge and comparable abundance are present. The ion trap offers the benefits of tandem mass spectrometry with little cost in signal or measurement rate. All parent ion types, $\mathbf{M}^{+}$, $(\mathrm{M}-\mathrm{H})^{+}$, and $\left(\mathrm{M}+\mathrm{NO}^{+}\right)$, yield high MS/MS efficiencies and the flexibility of the ion trap allows for parallel monitoring procedures to minimize potential losses in measurement rate associated with tandem mass spectrometry. The combination of sensitivity, speed, specificity, and flexibility afforded by the nitric oxide Cl/ion trap approach makes it an attractive option for engine exhaust measurements. 


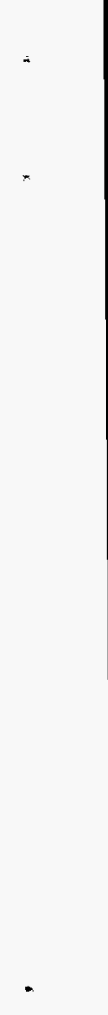




\section{INVENTIONS}

No patentable inventions were made during the course of this work. 



\section{COMMERCIALIZATION POSSIBILITIES}

There are currently no commercial vendors capable of supplying a copy of the final prototype instrument. Furthermore, further software development to integrate sampling, exhaust gas treatment, analysis, and data reduction is required for routine operation in an engine vehicle test environment. However, the ion trap system that constitutes the heart of the analyzer is a commercially available system and the same vendor (Teledyne Electronic Technologies, Mountain View, CA) also offers glow discharge ionization through its license with ORNL. Therefore, technology transfer involving McClellan, Ford, and Teledyne could yield a commercially available system. 


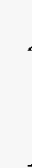




\section{FUTURE COLLABORATIONS}

There are no funded collaborative projects currently planned. However, this project established an excellent working relationship between the scientists at Ford and at ORNL. Dr. Dearth, for example, is regarded as a valuable resource in providing advice in the current project focused on the ionization and ion chemistry of oxygenated hydrocarbons. 


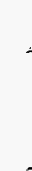




\section{CONCLUSIONS}

This project clearly established that ion trap mass spectrometry has many attractive features as a tool for measurement of automotive emissions from LEVs. In particular, it has been demonstrated that nitric oxide chemical ionization coupled with ion trap mass spectrometry is capable of monitoring multiple species of interest, including alkanes, alkenes, and arenes, at low parts per billion concentration levels at measurement rates greater than $1 \mathrm{~Hz}$. The measurements can be made with the high specificity ordinarily associated with mass spectrometry and, furthermore, can take advantage of the added specificity of tandem mass spectrometry without significant loss in either sensitivity or speed. This capability is made possible via the use of tailored wave-forms applied to the end-cap electrodes of the ion trap such that parallel monitoring schemes can be employed. Other forms of ionization, such as atmospheric sampling glow discharge ionization and in situ water chemical ionization, can also play roles in automotive emissions measurements, particularly with oxygenated hydrocarbons, arenes, and, perhaps, alkenes. It is also highly likely that other important exhaust components, such as sulfur dioxide, oxides of nitrogen, as well as other species not targeted in this work will be amenable to one or more of the approaches developed as part of this CRADA. Ion trap mass spectrometry, with its various ionization methods, is likely to be the most flexible, sensitive, and specific of all analytical tools considered for automotive emissions measurements. 


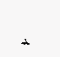




\section{ACKNOWLEDGMENT}

We acknowledge Mr. Dan Sheddy, Mr. Kirby Schlaht, Mr. Vinoo Jain and co-workers from McClellan Air Force Base for providing use of the Teledyne-Hitachi 3DQ and for fabricating the ion source and lens stack used to modify the ion trap for glow discharge ionization. 


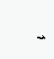




\section{REFERENCES}

1. D. L. Illman, C\&ENews, 1994, 72, 8-16.

2. D. Scheutzle, T.E. Jensen, D. Nagy, A. Prostak, and A. Hochhauser, Anal. Chem., 1991, 63, 1149A-1159A.

3. C. Weickhardt, U. Boesl, and E.W. Schlag, Anal. Chem., 1994, 66, 1062-1069.

4. M.A. Dearth, C.A. Gierczak, and W.O.S. Siegl, Environ. Sci. Technol., 1992, 26, 1573-1580.

5. N. Einolf and B. Munson, Int. J. Mass Spectrom. Ion Phys., 1972, 9, 141-160.

6. D.H. Hunt and J.F. Ryan, J. Chem. Soc. Chem. Commun., 1972, 620-621.

7. B.L. Jelius, B. Munson, and C. Fenselau, Biomed. Mass Spectrom., 1974, 1, 96102.

8. I. Jardine and C. Fenselau, Anal. Chem., 1975, 47, 730-733.

9. I. Jardine and C. Fenselau, Org. Mass Spectrom., 1975, 10, 748-751.

10. D.F. Hunt and T.M. Harvey, Anal. Chem., 1975, 47, 1965-1969.

11. D.F. Hunt and T.M. Harvey, Anal. Chem., 1975, 47, 2136-2141.

12. I. Dzidic, D.D. Carroll, R.N. Stillwell, and E.C. Horning, Anal. Chem., 1976, 48, 1763-1768.

13. E. Busker and H. Budzikiewicz, Org. Mass Spectrom., 1979, 13, 222-226.

14. D.F. Hunt, T.M. Harvey, W.C. Brumley, J.F. Ryan, and J.W. Russell, Anal. Chem., $1982,54,492-496$.

15. I. Dzidic, H.A. Peterson, P.A. Wadsworth, and H.V. Hart, Anal. Chem., 1992, 64, 2227-2232.

16. M.A. Dearth and T.J. Korniski, J. Am. Soc. Mass Spectrom., 1994, 5, 1107-1114.

17. M.B. Wise and M.R. Guerin, Anal. Chem., 1997, 69, 26A-32A.

18. K.J. Hart, A.B. Dindal, and R.R. Smith, Rapid Commun. Mass Spectrom., 1996, 10 , 352-360.

19. S.A. McLuckey, G.J. Van Berkel, D.E. Goeringer, and G.L. Glish, Anal. Chem., 1994, 66, 689A-696A, 737A-743A.

20. S.A. McLuckey, G.L. Glish, K.G. Asano, and B.C. Grant, Anal. Chem., 1988, 60, 2220-2227.

21. S.A. McLuckey, D.E. Goeringer, K.G. Asano, G. Vaidyanathan, and J. L. Stephenson, Jr., Rapid Commun. Mass Spectrom., 1996, 10, 287-298.

22. D.C. Duckworth, D.H. Smith, and S.A. McLuckey, J. Anal. At. Spectrom., 1997, 12, 43-48.

23. J.S. Brodbelt, J.N. Louris, and R.G. Cooks, Anal. Chem., 1987, 59, 1278-1285.

24. S.G. Lias, J.E. Bartmess, J.F. Leibman, J.L. Holmes, R.D. Levin, and W.G. Mallard, J. Phys. Chem Ref. Data, 1988, 17, Supplement 1.

25. S.G. Lias, H.M. Rosenstock, K. Draxl, B.W. Steiner, J.T. Herron, J.L. Holmes, R.D. Levin, J.F. Liebman, and S.A. Kafafi, in NIST Standard Reference Database Number 69, W.G. Mallard and P.J. Linstrom, Eds., February 1997, National Institute of Standards and Technology, (http://webbook.nist.gov).

26. J.N. Louris, J.W. Amy, T.Y. Ridley, and R.G. Cooks, Int. J. Mass Spectrom. Ion Processes, 1989, 88, 97-111. 
27. S.A. McLuckey, G.L. Glish, and K.G. Asano, Anal. Chim. Acta, 1989, 225, 25-35.

28. G.J. Van Berkel, S.A. McLuckey, and G.L. Glish, Anal. Chem., 1991, 63, 10981109.

29. S.A. McLuckey, D.E. Goeringer, and G.L. Glish, J. Am. Soc. Mass Spectrom.,1991, 2, 11-21.

30. D.E. Goeringer, K.G. Asano, S.A. McLuckey, D. Hoekman, and S.E. Stiller, Anal. Chem., 1994, 66, 313-318.

31. R.E. March and R.J. Hughes, "Quadrupole Storage Mass Spectrometry," John Wiley, New York, 1989.

32. M. Weber-Grabau, S.C. Bradshaw, and J.E.P. Syka, U.S. Patent No. 4,771,172, 1987.

33. K.L. Busch and R.G. Cooks, in "Tandem Mass Spectrometry," McLafferty (Ed.), Wiley, New York, 1983, Chapter 2.

34. K.L. Busch, G.L. Glish, and S.A. McLuckey, "Mass Spectrometry/Mass Spectrometry: Techniques and Applications of Tandem Mass Spectrometry," VCH Publishers, New York, 1988.

35. J.N. Louris, R.G. Cooks, J.E.P. Syka, P.E. Kelley, G.C. Stafford, Jr., and J.F.J. Todd, Anal. Chem., 1987, 59, 1677-1685.

36. R.E. March, R.J. Strife, and C.S. Creaser, in "Practical Aspects of Ion Trap Mass Spectrometry, Volume III," R.E. March and J.F.J. Todd, (Eds.), CRC Press, Boca Raton, 1995, Chapter 2.

37. R.K. Julian, Jr., K. Cox, and R.G. Cooks, Anal. Chem., 1993, 65, 1827-1833.

38. K.G. Asano, D.E. Goeringer, and S.A. McLuckey, Anal. Chem., 1995, 67, 27392742.

39. P.E. Kelley, U.S. Patent 5,135,286, 1992. 


\section{LIST OF TABLES}

Table 1. Operating conditions used for ion formation, accumulation, and detection

Table 2. Reagent gas and analyte (species in gas mix standard) data

Table 3. Parent and product ions observed for the gas mix standard. In cases in which two product ions are indicated, the underlined ion was the most abundant. When the two major product ions were of similar abundance, neither product ion is underlined. 
Table 1. Operating conditions used for ion formation, accumulation, and detection

\begin{tabular}{|l|l|}
\hline Glow discharge source pressure & $0.8-1.4$ torr \\
\hline Source gas composition & $\begin{array}{l}0.2-0.5 \text { torr } 1-10 \% \text { NO in } \mathrm{N}_{2}, \\
0.6-0.9 \text { torr sample gas }\end{array}$ \\
\hline Vacuum system pressure & $1 \times 10^{-4}$ torr (no helium) \\
& $1-1.5 \times 10^{-3}$ torr (helium added) \\
\hline Source sampling rate & $15 \mathrm{ml} / \mathrm{min}$ \\
\hline Sampling capillary & $-350--450 \mathrm{~V}$ during discharge \\
\hline Half-plate & $+8-+15 \mathrm{~V}$ \\
\hline discharge current & $0.25-0.5 \mathrm{~mA}$ \\
\hline L1 & $-10--30 \mathrm{~V}$ \\
\hline L2 & $-100--170 \mathrm{~V}$ \\
\hline L3 & $-300--1000 \mathrm{~V}$ \\
\hline Electron multiplier & $-1800 \mathrm{~V}$ \\
\hline
\end{tabular}


Table 2. Reagent gas and analyte (species in gas mix standard) data

\begin{tabular}{|c|c|c|c|c|c|}
\hline Compound & $\begin{array}{l}\text { Cylinder } \\
\text { conc. } \\
\text { (ppm) }\end{array}$ & mass & $\begin{array}{c}\text { Ionization } \\
\text { energy } \\
(\mathrm{eV})\end{array}$ & $\begin{array}{c}\text { Proton } \\
\text { affinity } \\
\text { (kcal/mol) }\end{array}$ & $\begin{array}{l}\text { Hydride ion } \\
\text { affinity }\left(\mathrm{R}^{+}\right) \\
(\mathrm{kcal} / \mathrm{mol})^{\dagger}\end{array}$ \\
\hline ethene & 2.5 & 28 & 10.5 & 162.6 & 287 \\
\hline propene & 1.7 & 42 & 9.7 & 179.6 & 254.5 \\
\hline 1,3-butadiene & 1.3 & 54 & 9.1 & 187.1 & 253 \\
\hline 1-butene & 1.3 & 56 & 9.6 & NA & $236-265$ \\
\hline 2-methyl-1,3-butadiene & 1.0 & 68 & 8.9 & 198.9 & $235-249$ \\
\hline cis-2-pentene & 1.0 & 70 & 8.9 & NA & $230-240$ \\
\hline trimethylethylene & 1.0 & 70 & 8.7 & 193.3 & $233-243$ \\
\hline isopentane & 1.0 & 72 & 10.2 & NA & 231 \\
\hline 2-methylpentane & 0.9 & 86 & 10.0 & NA & $225-245$ \\
\hline 3-methylpentane & 0.9 & 86 & 10.0 & NA & 224-244 \\
\hline 2,3-dimethylpentane & 0.8 & 100 & NA & NA & NA \\
\hline isooctane & 0.7 & 114 & 9.9 & NA & 226 \\
\hline benzene & 0.9 & 78 & 9.24 & 179.4 & 283 \\
\hline toluene & 0.8 & 92 & 8.8 & 187.4 & 237 \\
\hline ethylbenzene & 0.7 & 106 & 8.8 & 188.3 & 225 \\
\hline o-xylene & 0.7 & 106 & 8.8 & 190 & 232 \\
\hline 1,2,4-trimethylbenzene & 0.6 & 120 & 8.3 & NA & 233 \\
\hline 1,3,5-trimethylbenzene & 0.6 & 120 & 8.4 & 200 & 233 \\
\hline 1-ethyl-2-methylbenzene & 0.6 & 120 & NA & NA & 233 \\
\hline methyl-t-butyl ether & 1.0 & 88 & 9.24 & 200.6 & 202 \\
\hline water & & 18 & 12.6 & 165 & 400 \\
\hline nitric oxide & & 30 & 9.26 & 109 & 246 \\
\hline
\end{tabular}

${ }^{\dagger}$ Estimates of hydride ion affinities calculated from heats of formation of the neutral molecules, cations formed by hydride abstraction, and the hydride anion. These values are only crude estimates in cases in which the structure of the cation is uncertain. 
Table 3. Parent and product ions observed for the gas mix standard. In cases in which two product ions are indicated, the underlined ion was the most abundant. When the two major product ions were of similar abundance, neither product ion is underlined.

\begin{tabular}{|c|c|c|c|}
\hline Compound & Parent ion & $\mathrm{m} / \mathrm{z}$ & Major product ions \\
\hline isopentane & $(\mathrm{M}-\mathrm{H})^{+}$ & 71 & $43\left(\mathrm{C}_{3} \mathrm{H}_{7}^{+}\right)$ \\
\hline 2-methyl pentane & $(\mathrm{M}-\mathrm{H})^{+}$ & 85 & $\begin{array}{l}43\left(\mathrm{C}_{3} \mathrm{H}_{7}^{+}\right) \\
41\left(\mathrm{C}_{3} \mathrm{H}_{5}^{+}\right)\end{array}$ \\
\hline 3-methylpentane & $(\mathrm{M}-\mathrm{H})^{+}$ & 85 & $57\left(\mathrm{C}_{4} \mathrm{H}_{9}^{+}\right)$ \\
\hline $\begin{array}{l}\text { 2,3- } \\
\text { dimethylpentane }\end{array}$ & $(\mathrm{M}-\mathrm{H})^{+}$ & 99 & $57\left(\mathrm{C}_{4} \mathrm{H}_{9}^{+}\right)$ \\
\hline isooctane & $(\mathrm{M}-\mathrm{H})^{+}$ & 113 & $\begin{array}{l}71\left(\mathrm{C}_{5} \mathrm{H}_{11}{ }^{+}\right) \\
\underline{57}\left(\mathrm{C}_{4} \mathrm{H}_{9}{ }^{+}\right)\end{array}$ \\
\hline 1,3-butadiene & $(\mathrm{M}+\mathrm{NO})^{+}$ & 84 & $54\left(M^{+}\right)$ \\
\hline 1-butene & $(\mathrm{M}+\mathrm{NO})^{+}$ & 86 & $56\left(M^{+}\right)$ \\
\hline $\begin{array}{l}\text { 2-methyl-1,3- } \\
\text { butadiene }\end{array}$ & $(\mathrm{M}+\mathrm{NO})^{+}$ & 98 & $68\left(\mathrm{M}^{+}\right)$ \\
\hline cis-2-pentene & $\begin{array}{c}(\mathrm{M}-\mathrm{H})^{+} \\
\mathrm{M}^{+} \\
(\mathrm{M}+\mathrm{NO})^{+}\end{array}$ & $\begin{array}{r}69 \\
70 \\
100\end{array}$ & $\begin{array}{l}41\left(\mathrm{C}_{3} \mathrm{H}_{5}^{+}\right) \\
55\left(\mathrm{C}_{4} \mathrm{H}_{7}^{+}\right) \\
\frac{41}{70}\left(\mathrm{C}_{3} \mathrm{H}_{5}^{+}\right) \\
70\left(\mathrm{M}^{+}\right)\end{array}$ \\
\hline trimethylethylene & $\begin{array}{c}(\mathrm{M}-\mathrm{H})^{+} \\
\mathrm{M}^{+} \\
(\mathrm{M}+\mathrm{NO})^{+}\end{array}$ & $\begin{array}{r}69 \\
70 \\
100\end{array}$ & $\begin{array}{c}41\left(\mathrm{C}_{3} \mathrm{H}_{5}^{+}\right) \\
55\left(\mathrm{C}_{4} \mathrm{H}_{7}^{+}\right) \\
41\left(\mathrm{C}_{3} \mathrm{H}_{5}^{+}\right) \\
70\left(\mathrm{M}^{+}\right)\end{array}$ \\
\hline benzene & $(\mathrm{M}+\mathrm{NO})^{+}$ & 108 & $78\left(M^{+}\right)$ \\
\hline toluene & $(\mathrm{M}+\mathrm{NO})^{+}$ & 122 & $92\left(\mathrm{M}^{+}\right)$ \\
\hline $\mathrm{C}_{2}$-benzenes & $(\mathrm{M}+\mathrm{NO})^{+}$ & 136 & $106\left(\mathrm{M}^{+}\right)$ \\
\hline $\mathrm{C}_{3}$-benzenes & $(\mathrm{M}+\mathrm{NO})^{+}$ & 150 & $120\left(\mathrm{M}^{+}\right)$ \\
\hline
\end{tabular}





\section{LEGENDS FOR FIGURES}

Figure 1 Side-view schematic of a glow discharge ionization source interfaced with a Teledyne 3DQ quadrupole ion trap mass spectrometer.

Figure 2 (a) Ion trap in situ water chemical ionization mass spectrum of the standard gas mixture. (b) Atmospheric sampling glow discharge mass spectrum of the standard gas mixture (no nitric oxide added). (c) Glow discharge nitric oxide chemical ionization mass spectrum of the standard gas mixture (low massto-charge cutoff $=9$ ).

Figure 3 Glow discharge nitric oxide chemical ionization mass spectrum of the standard gas mixture using a low mass-to-charge cutoff of 24 .

Figure 4 (a) Glow discharge nitric oxide Cl mass spectrum acquired from the undiluted gas mixture using a $100 \mathrm{~ms}$ ion accumulation period. (b) Mass spectrum obtained under identical conditions with the gas mixture diluted 67-fold to yield mixture component concentrations of roughly $15 \mathrm{ppb}$.

Figure 5 Peak area versus concentration curves for the molecular ions of the pentenes at $\mathrm{m} / \mathrm{z} 70$, the $(M-H)^{+}$of isopentane at $\mathrm{m} / \mathrm{z} 71$, and the $\left(M+\mathrm{NO}^{+}\right)$ion of benzene at $\mathrm{m} / \mathrm{z} 108$.

Figure 6 (a) Isolation of the molecular ions of the pentanes. (b) Product ions obtained from ion trap collisional activation of the $\mathrm{m} / \mathrm{z} 70$ parent ions of (a) for $20 \mathrm{~ms}$.

Figure 7 (a) Mass spectrum resulting from the use of a filtered-noise field to selectively isolate the $\left(\mathrm{M}+\mathrm{NO}^{+}\right)$ions of butadiene, 1,3-butadiene, benzene, toluene, the $\mathrm{C}_{2}$-benzenes, and the $\mathrm{C}_{3}$-benzenes. (b) Spectrum that results when a second filtered-noise field designed to collisionally activate the $\left(\mathrm{M}+\mathrm{NO}^{+}\right)$ions of (a) is applied prior to the analytical scan. 


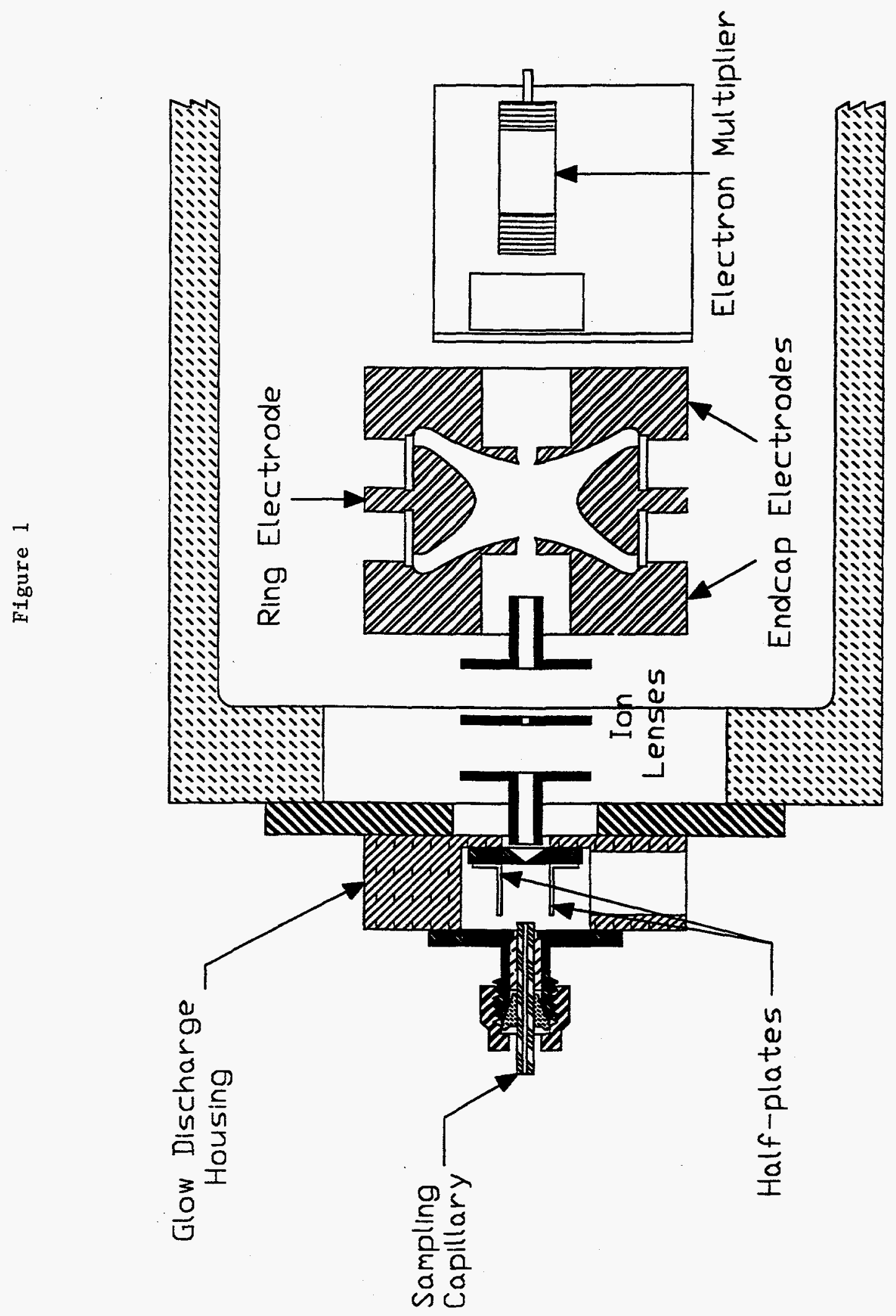




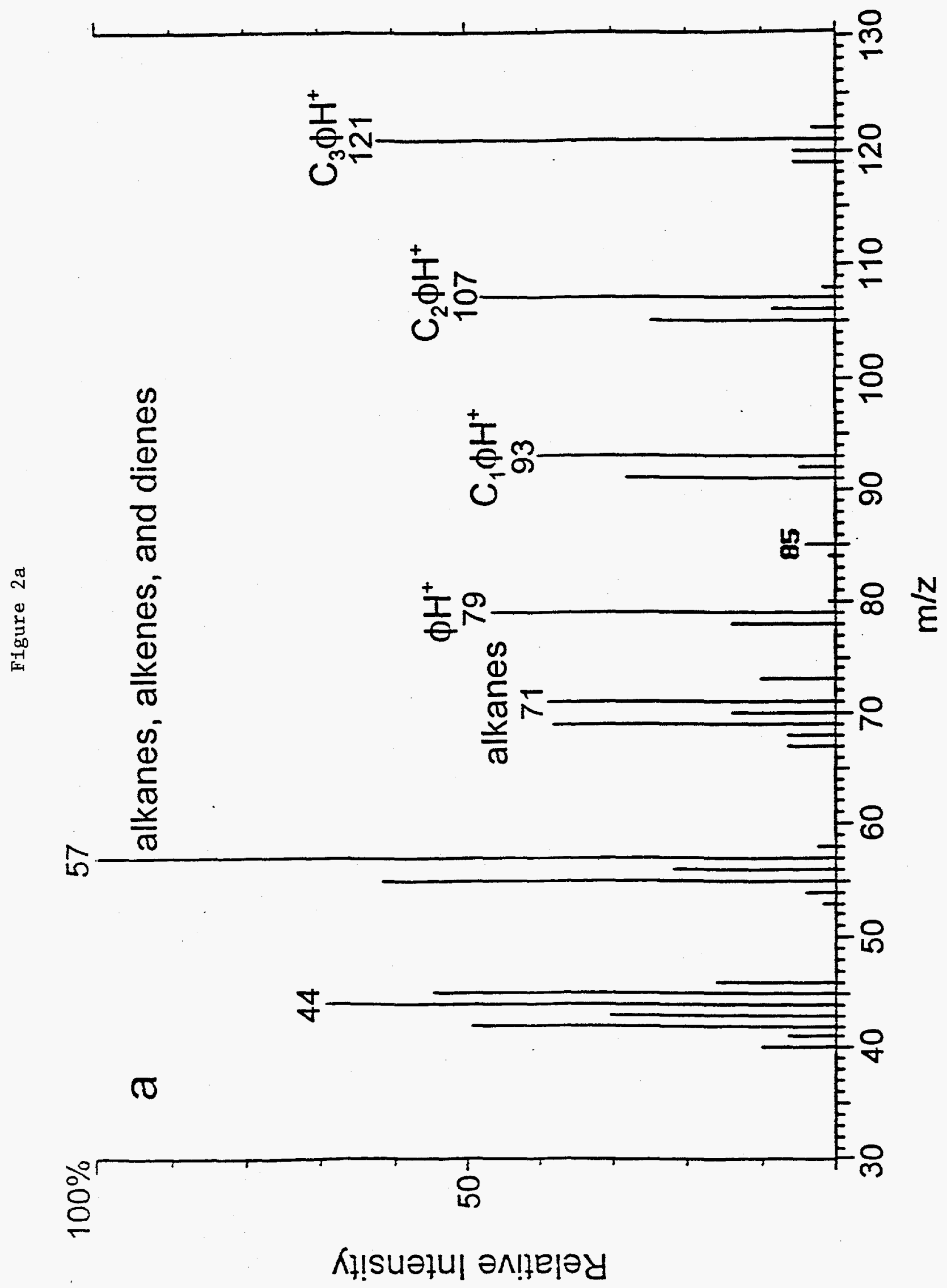




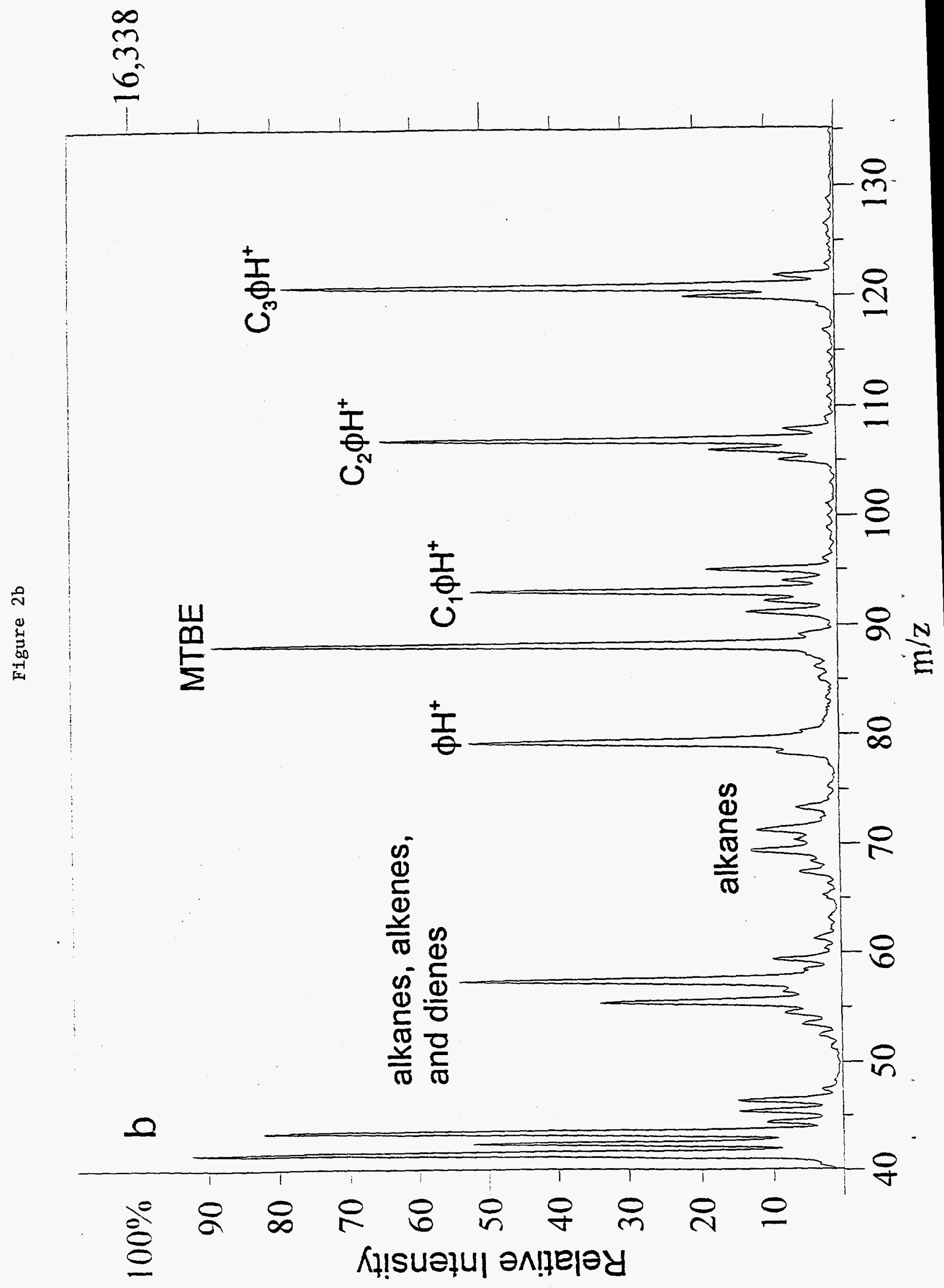




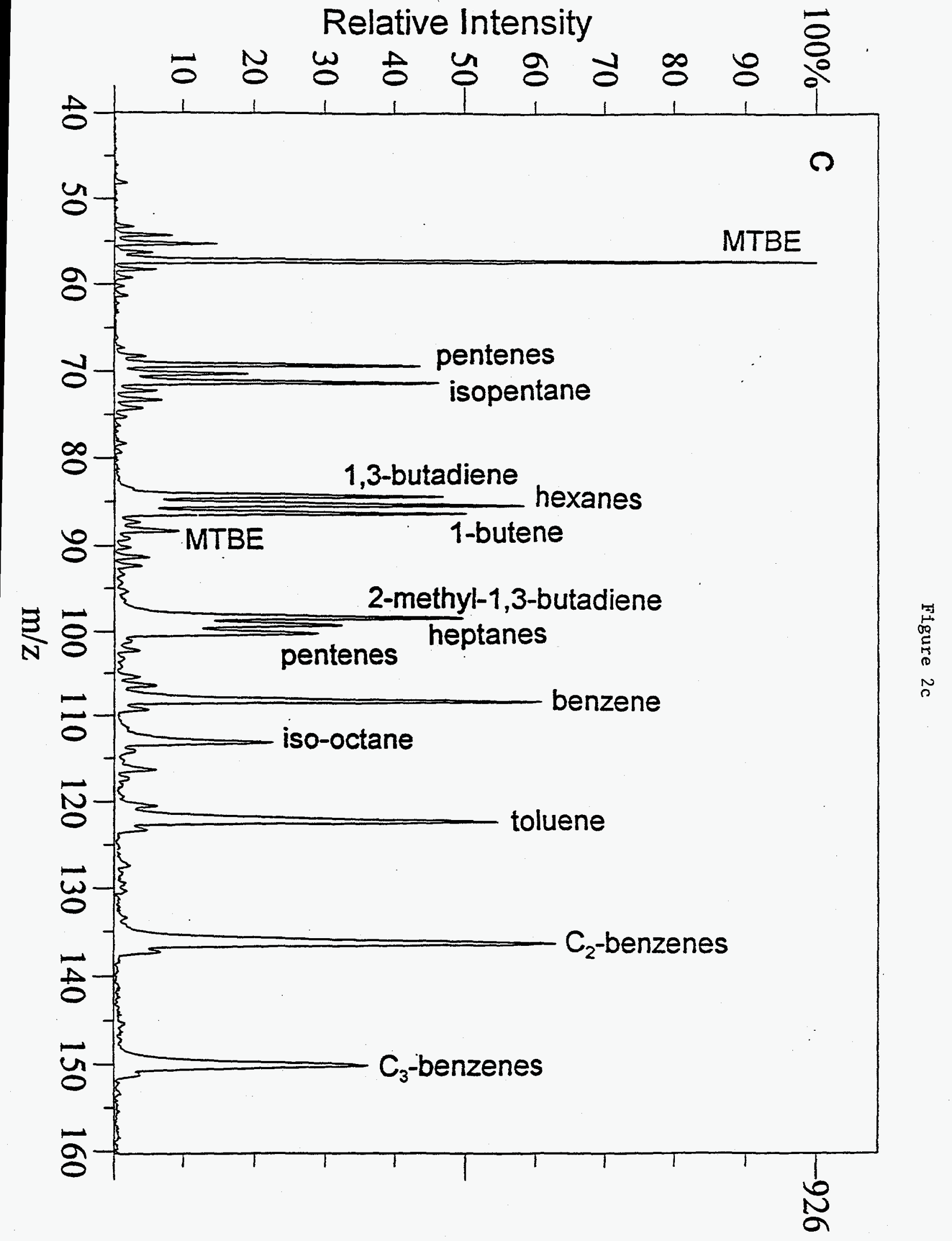




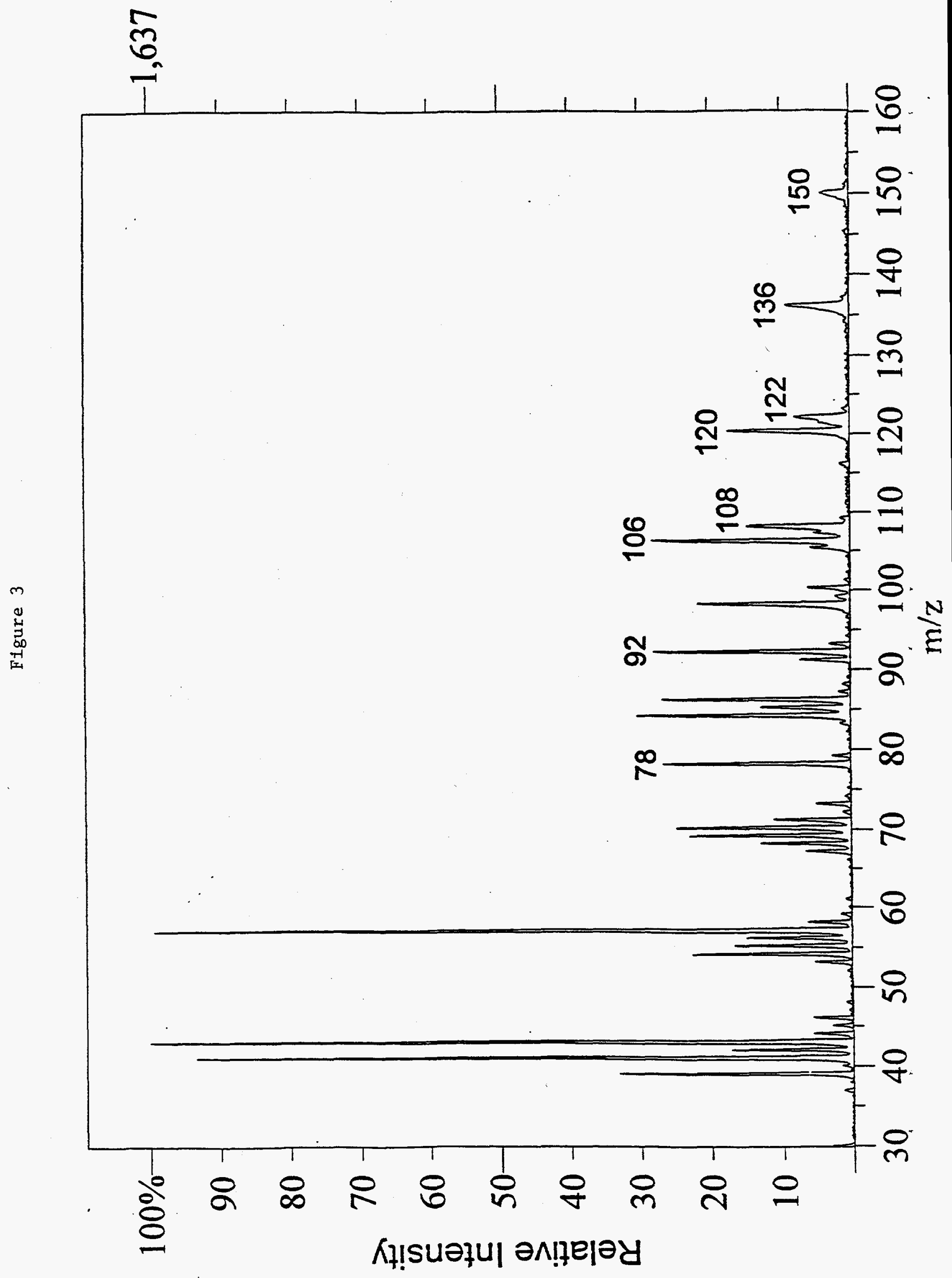



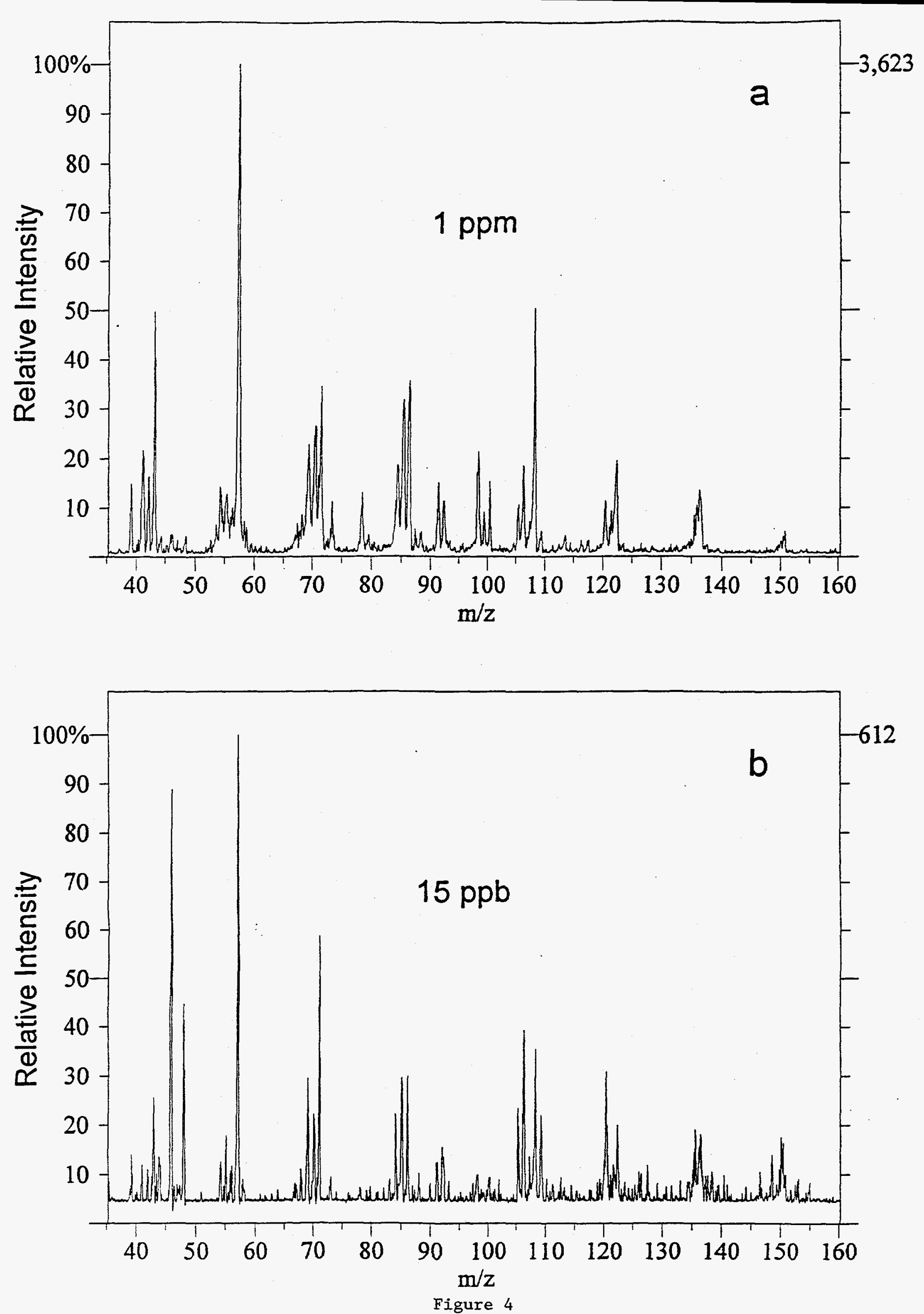


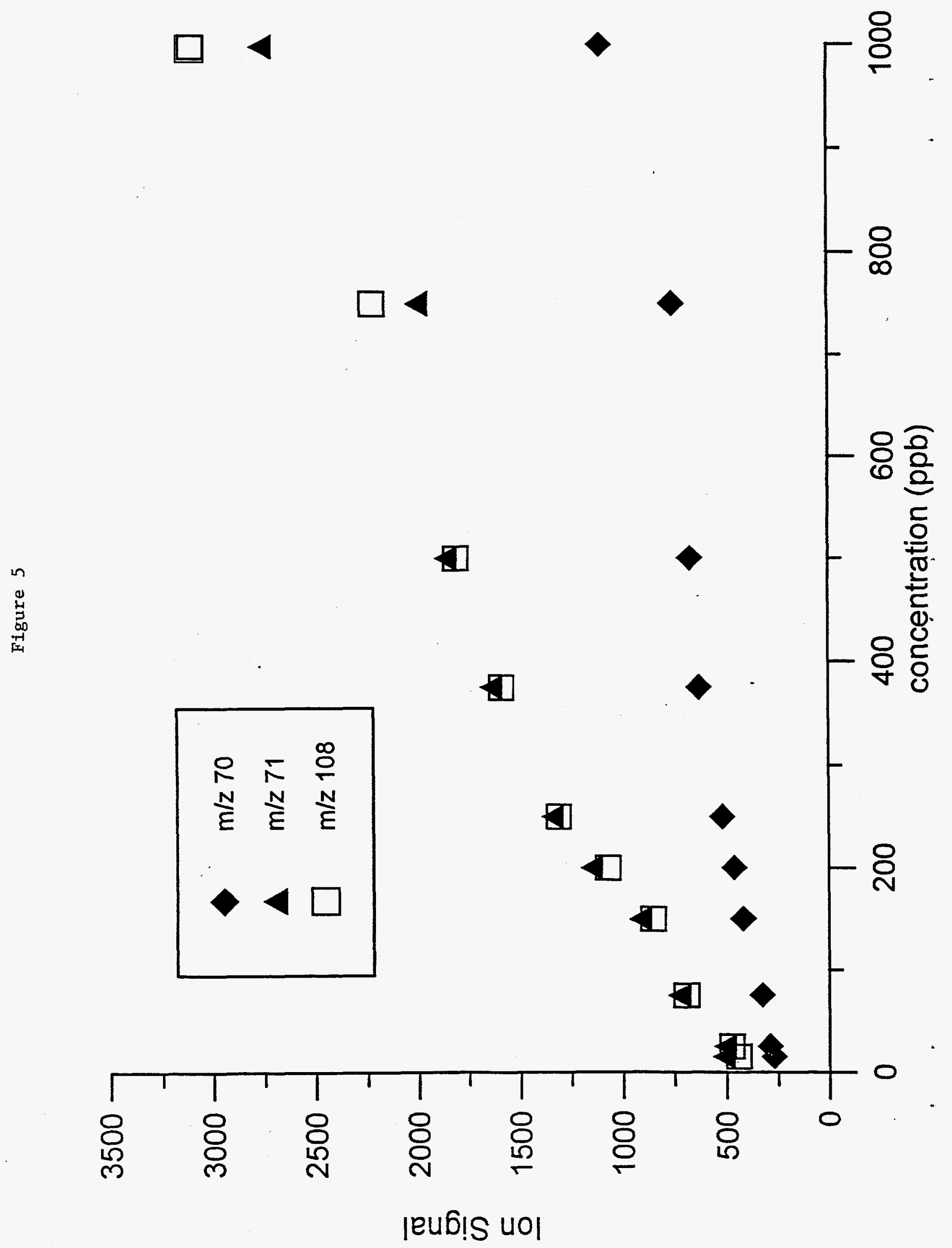



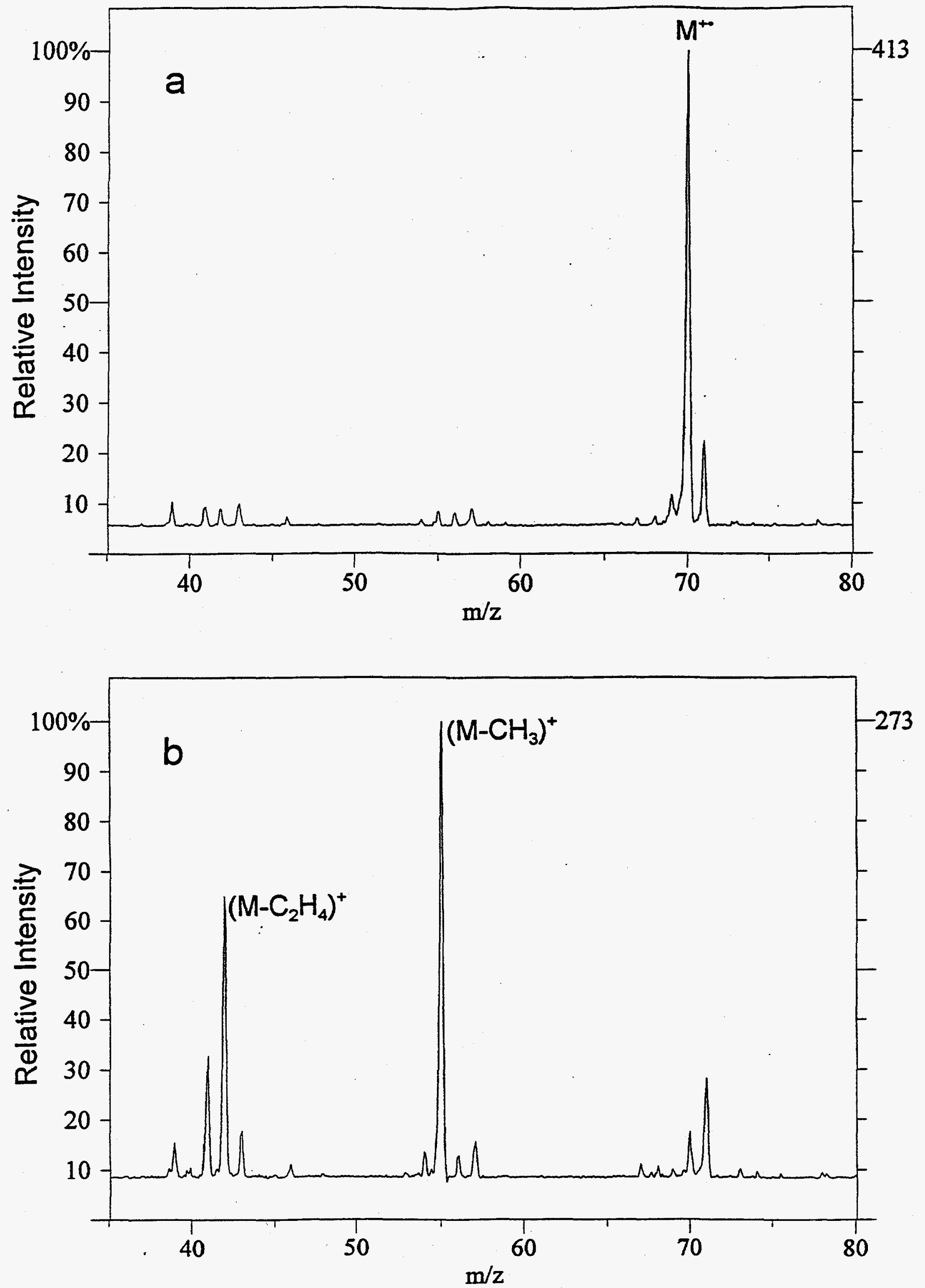

Figure 6 

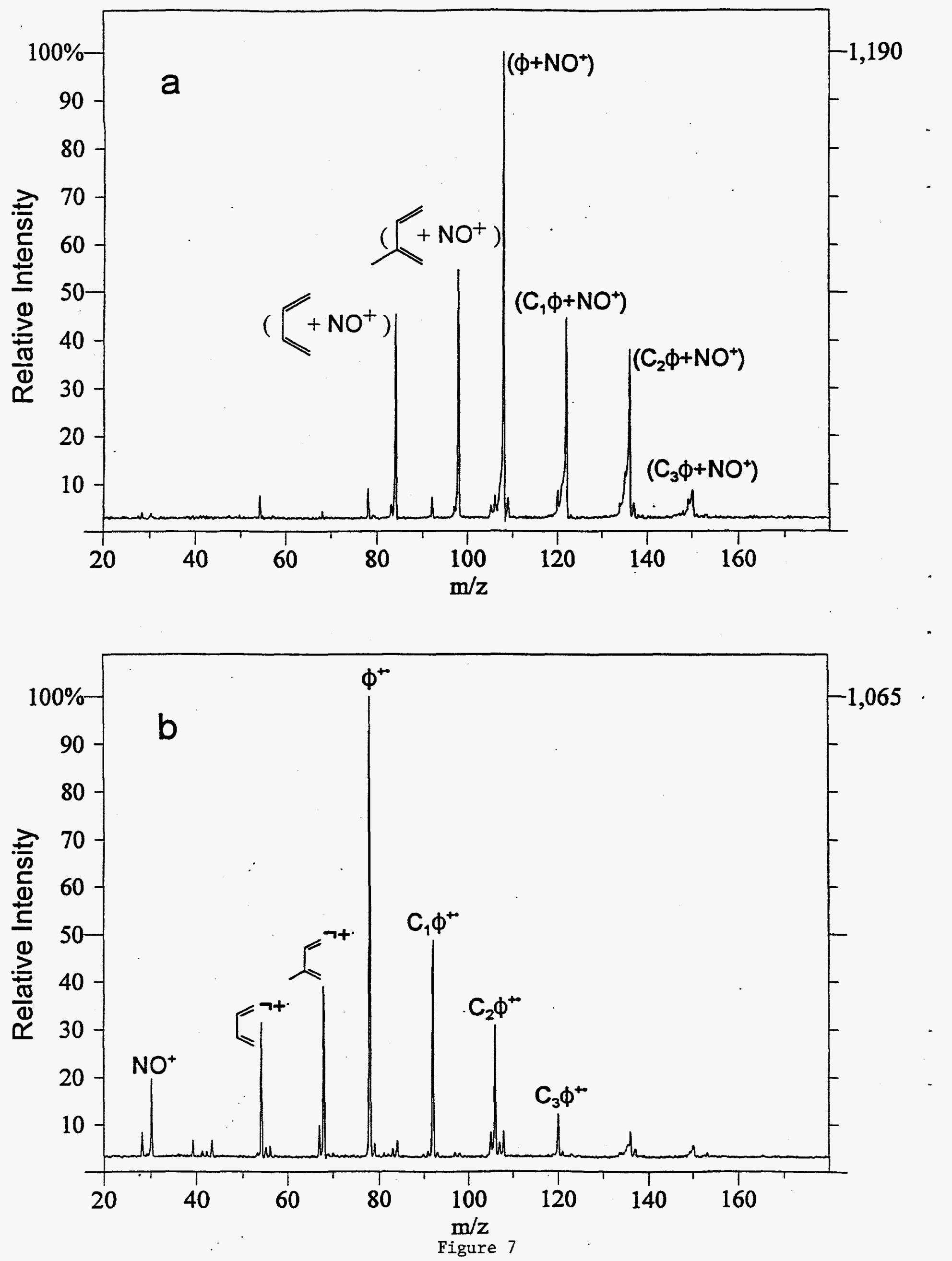


\section{APPENDIX 1 - Ion Trap in situ Water Chemical Ionization}

\section{Introduction}

In-situ water $\mathrm{Cl}$ was investigated as a potential method for the determination of several oxygenated species in auto exhaust at the low ppb level. One of the reasons that $\mathrm{NO}^{+}$attachment was not desirable for determination of formaldehyde was that it was difficult to distinguish formaldehyde from $\mathrm{NO}^{+}$(i.e., formaldehyde and $\mathrm{NO}^{+}$are both $30 \mathrm{Da}$ ). Significant peaks from the major constituents of air at $\mathrm{m} / \mathrm{z} 28$ and $\mathrm{m} / \mathrm{z} 32$ rule out quantitating methanol and formaldehyde by $E I(\mathrm{~m} / \mathrm{z} 32$ and $\mathrm{m} / \mathrm{z} 30)$. Since the listed oxygenated species accept a proton from water while oxygen and nitrogen do not, this method has the potential of eliminating the major interfering ions while yielding unique ions for quantitation. Some useful reference data are summarized below.

$$
\text { quantitation ions }\left([\mathrm{M}+\mathrm{H}]^{+}\right)
$$

$\begin{array}{lcc}\begin{array}{l}\text { ethanol } \\ \text { acetaldehyde }\end{array} & m / 247 \\ \begin{array}{l}\text { methanol } \\ \text { formaldehyde }\end{array} & m / 233 & \\ & m / z 31 & \\ & \text { PA (kcal/mol) }{ }^{1} & \text { IP (eV) } \\ \mathrm{O}_{2} & 101.5 & 12.06 \\ \mathrm{~N}_{2} & 118.2 & 15.58 \\ \mathrm{H}_{2} \mathrm{O} & 166.5 & 12.6 \\ \text { formaldehyde } & 171.7 & \\ \text { methanol } & 181.9 & \\ \text { acetaldehyde } & 186.6 & \\ \text { ethanol } & 188.3 & \end{array}$

\section{Experimental}

The data presented here were obtained using a Finnigan ITMS instrument and a direct sampling air monitor interface. The air monitor combines sample gas with helium and introduces this mixture into the ion trap via an open split capillary transfer line. ${ }^{2}$ Low $\mathrm{ppb}$ detection limits have been obtained using this interface in the El mode for several VOCs in air. ${ }^{3}$

\section{Results and Discussion}

One of the first problems encountered when using water $\mathrm{Cl}$ for analytes in the $\mathrm{m} / \mathrm{z}$ 28 to $\mathrm{m} / \mathrm{z} 33$ region is the appearance of oxygen ions via charge exchange with $\mathrm{H}_{2} \mathrm{O}^{+}$. If 
all non-reagent, El generated ions are ejected from the trap and $\mathrm{H}_{3} \mathrm{O}^{+}$is isolated from $\mathrm{H}_{2} \mathrm{O}^{+}$. (hence the use of SRICl) then the interference from the air peaks was eliminated. The water $\mathrm{SRICl}$ mass spectrum of formaldehyde is characterized primarily by the $[\mathrm{M}+\mathrm{H}]^{+}$ion at $\mathrm{m} / \mathrm{z} 31$ as shown in Fig. A1/1a, although some $\mathrm{m} / \mathrm{z} 29$ and $\mathrm{m} / \mathrm{z} 30$ is also observed. The water $S R I C l$ mass spectrum of methanol is characterized primarily by the $[\mathrm{M}+\mathrm{H}]^{+}$ion at $\mathrm{m} / \mathrm{z}$ 33 as shown in Fig. A1/1b. An approximately equal mixture of formaldehyde and methanol was analyzed using water $\mathrm{SRICl}$ and the resulting spectrum is shown in Fig. A1/1c. The MS/MS spectrum of the $[M+H]^{+}$ion of formaldehyde shows primarily loss of $\mathrm{H}_{2}$ at $m / z 29$, as shown in Fig. A1/2.

One experiment where a 5 ppbv methanol standard in air was analyzed yielded a $\mathrm{s} / \mathrm{b}$ of $\sim 9$. The mass profile for $\mathrm{m} / \mathrm{z} 33$ (methanol) obtained from this experiment is shown in Fig. A1/3. This result was difficult to obtain on the ITMS and it was never reproduced on the Teledyne 3DQ, although several attempts were made to do so.

The reason water $\mathrm{Cl}$ does not yield low detection limits for air samples remains unclear. Water $\mathrm{Cl}$ has yielded low ppb detection limits for many VOCs including oxygenated species in water purge experiments (e.g., methyl ethyl ketone, $3.2 \mathrm{ppb}$ ). ${ }^{4}$ The water purge experiment is conducted by bubbling helium in a $40 \mathrm{~mL}$ vial containing the water sample. The helium and any partitioned VOCs are swept out of the vial and into an open-split capillary transfer line which is connected to the ion trap vacuum system. The general observation in the water purge experiment is that any headspace in the vial above the water level must be purged out of the vial before optimal mass resolution and sensitivity for the purged VOCs are obtained.

Lammert and co-workers have shown that the optimal $q_{\text {eject }}$ changes when air is used as a buffer gas instead of helium. ${ }^{5}$ So at least part of the explanation for the relative insensitivity of water $\mathrm{Cl}$ for air samples may be that the partial pressure of air from the sampling interface is large enough to change the optimal $q_{\text {eject. }}$. It is difficult to imagine that this phenomenon explains the problem given that a relatively large reagent ion abundance is observed while sampling air via the air module. The possibility exists that the presence of air impedes the proton transfer reaction in some fashion. Experiments to systematically investigate the effect of $\mathrm{N}_{2}$ and $\mathrm{O}_{2}$ at various partial pressures in an ion trap while performing in situ water $\mathrm{Cl}$ have been proposed. ${ }^{6}$

\section{Conclusions}

In situ water $\mathrm{Cl}$ did not yield sufficient sensitivity for the targeted oxygenated species in air samples to warrant the development of an analytical method. The reason for this finding remains unclear. A systematic investigation of the role air plays in the in situ chemical ionization experiment should clarify the situation and possibly lead to a basis for selecting an alternate $\mathrm{Cl}$ reagent. 


\section{Appendix 1 Figure Legends}

A1/1. Water SRICl mass spectra of (a) formaldehyde, (b) methanol and (c) a 50/50 mixture of formaldehyde and methanol.

$\mathrm{A} 1 / 2$. MS/MS spectrum of the $[\mathrm{M}+\mathrm{H}]^{+}$ion of methanol formed by water $\mathrm{Cl}$.

A1/3. Mass profile of $\mathrm{m} / \mathrm{z} 33$, the $[\mathrm{M}+\mathrm{H}]+$ ion for methanol, formed by water $\mathrm{SRICl}$ of a 5 ppbv methanol standard in ambient air.

\section{Appendix 1 References}

1. A.G. Harrison, Chemical Ionization Mass Spectrometry, CRC Press: Boca Raton, FL, 1983.

2. M.B. Wise and M.R. Guerin, Anal. Chem., 1997, 69, 26A-32A.

3. K.J. Hart, Proceedings of the 43rd ASMS Conference on Mass Spectrometry \& Allied Topics, Atlanta, GA, May 21-26, 1995, p. 1110.

4. M.B. Wise, M.V. Buchanan, M.R. Guerin, R. Merriweather, C.K. Bayne, Proceedings of the 41st ASMS Conference on Mass Spectrometry \& Allied Topics, San Francisco, CA, May 31-June 4, 1993, p. 126a.

5. S.A. Lammert and J.M. Wells, Rapid Commun. Mass Spectrom., 1996, 10, 361-71.

6. K.J. Hart, contained in an FY 1998 EMSP proposal. 


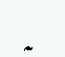


A)

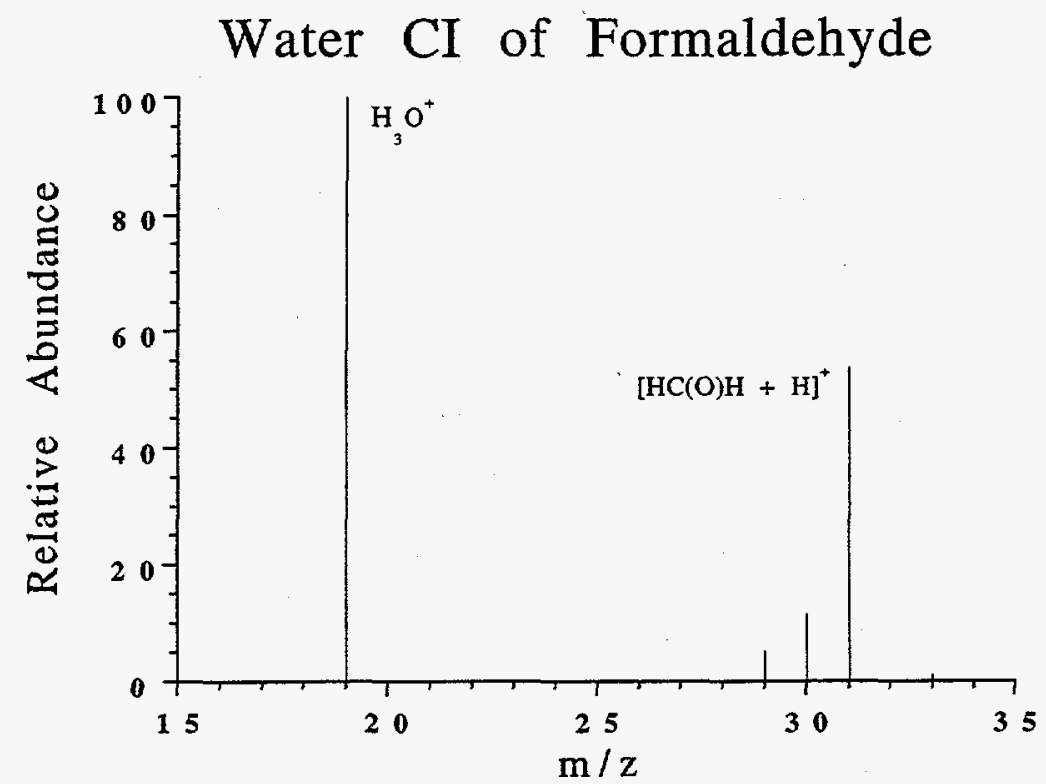

B)

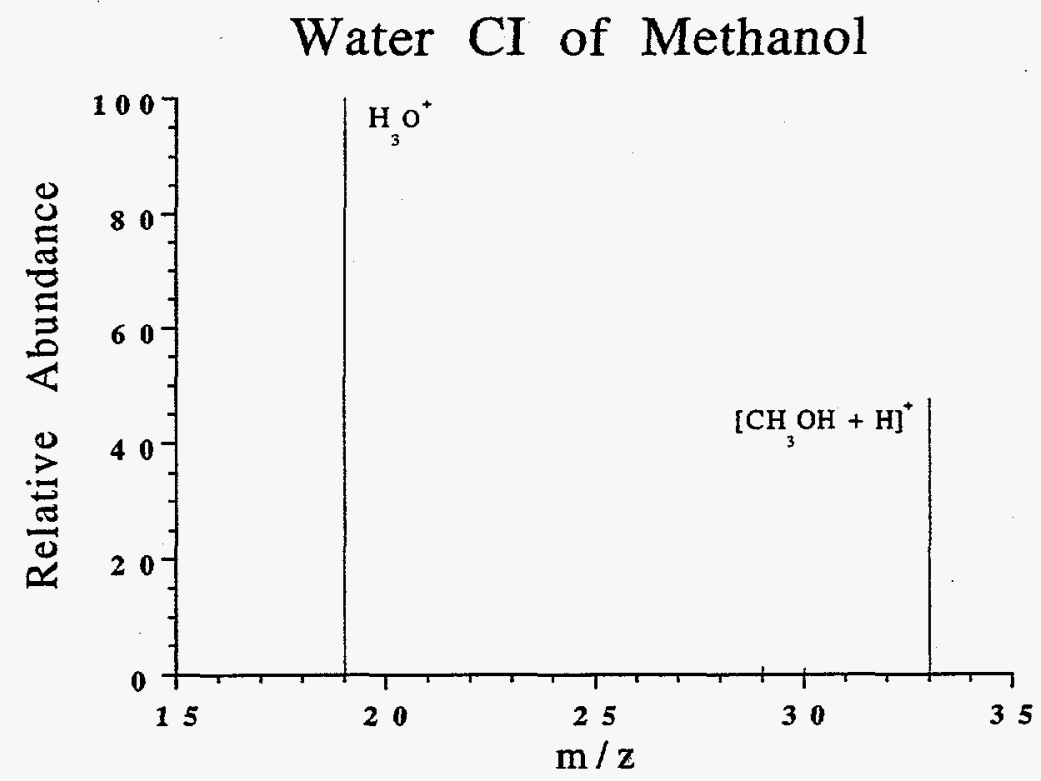

C)

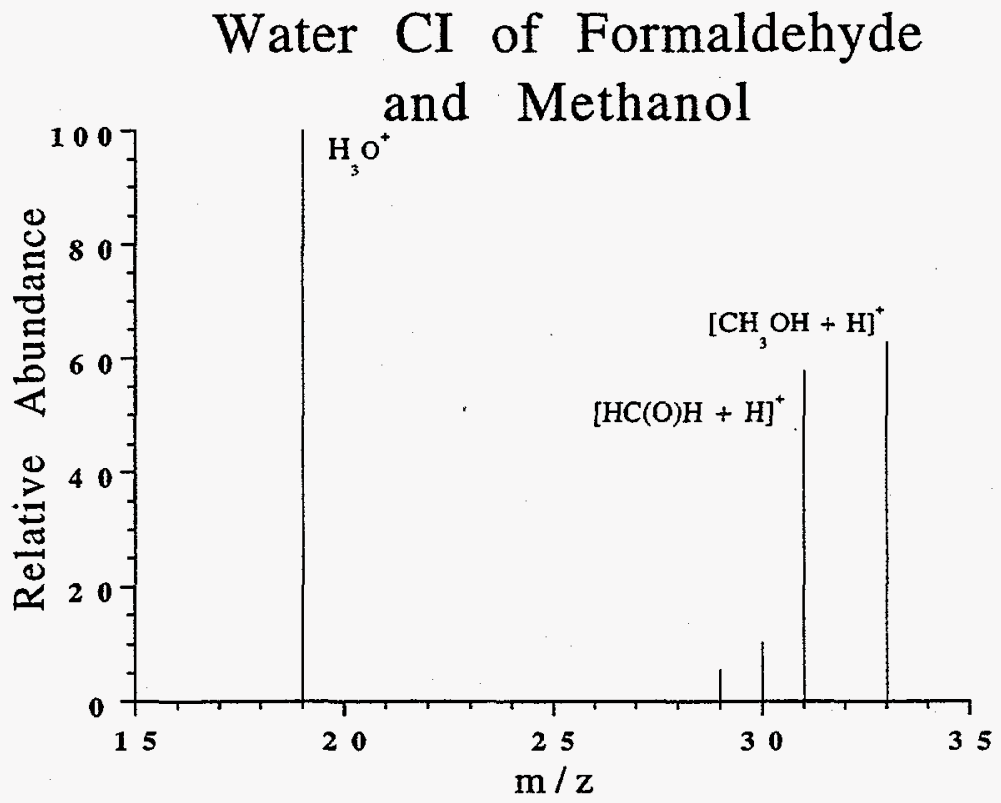




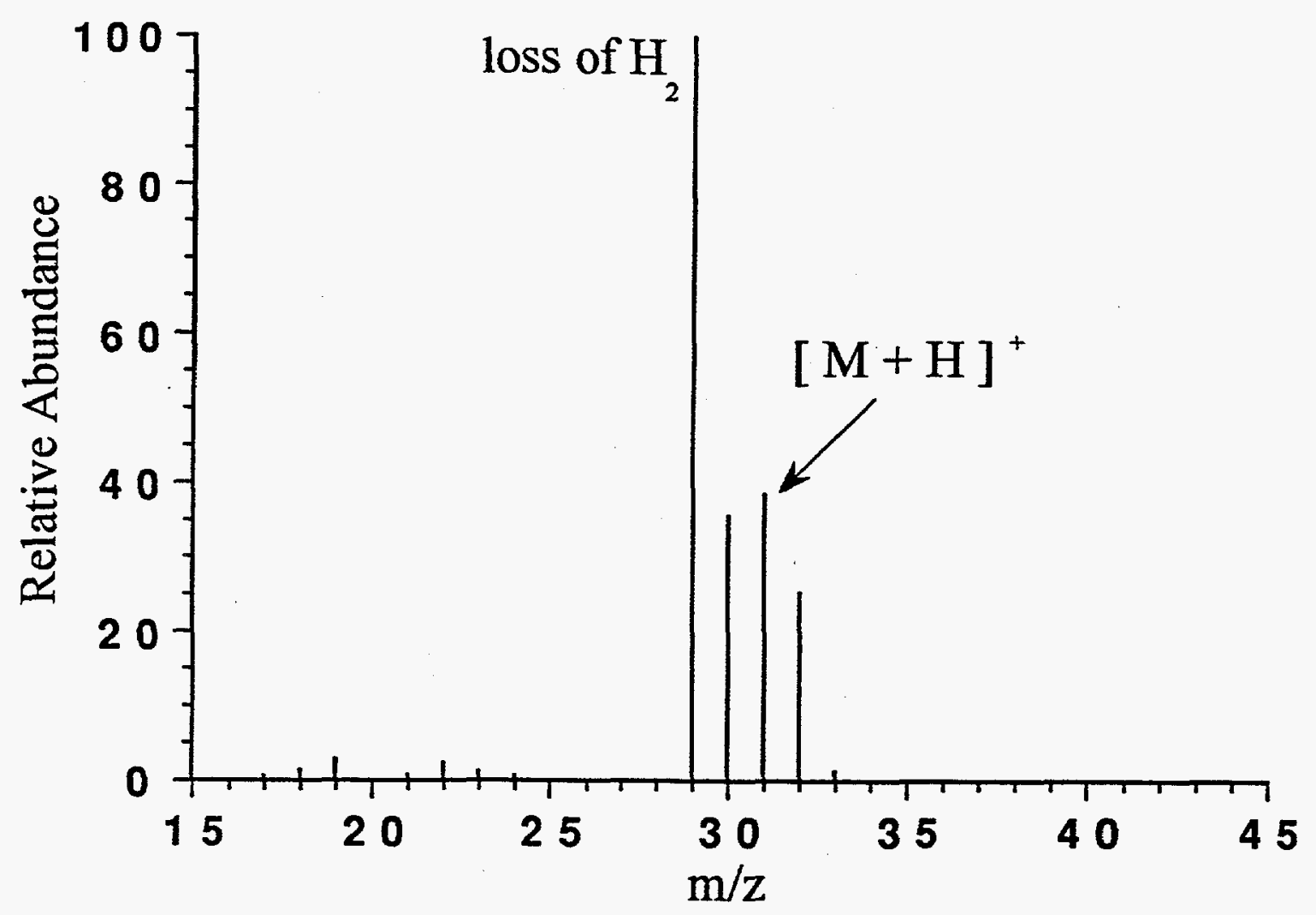

Figure 2

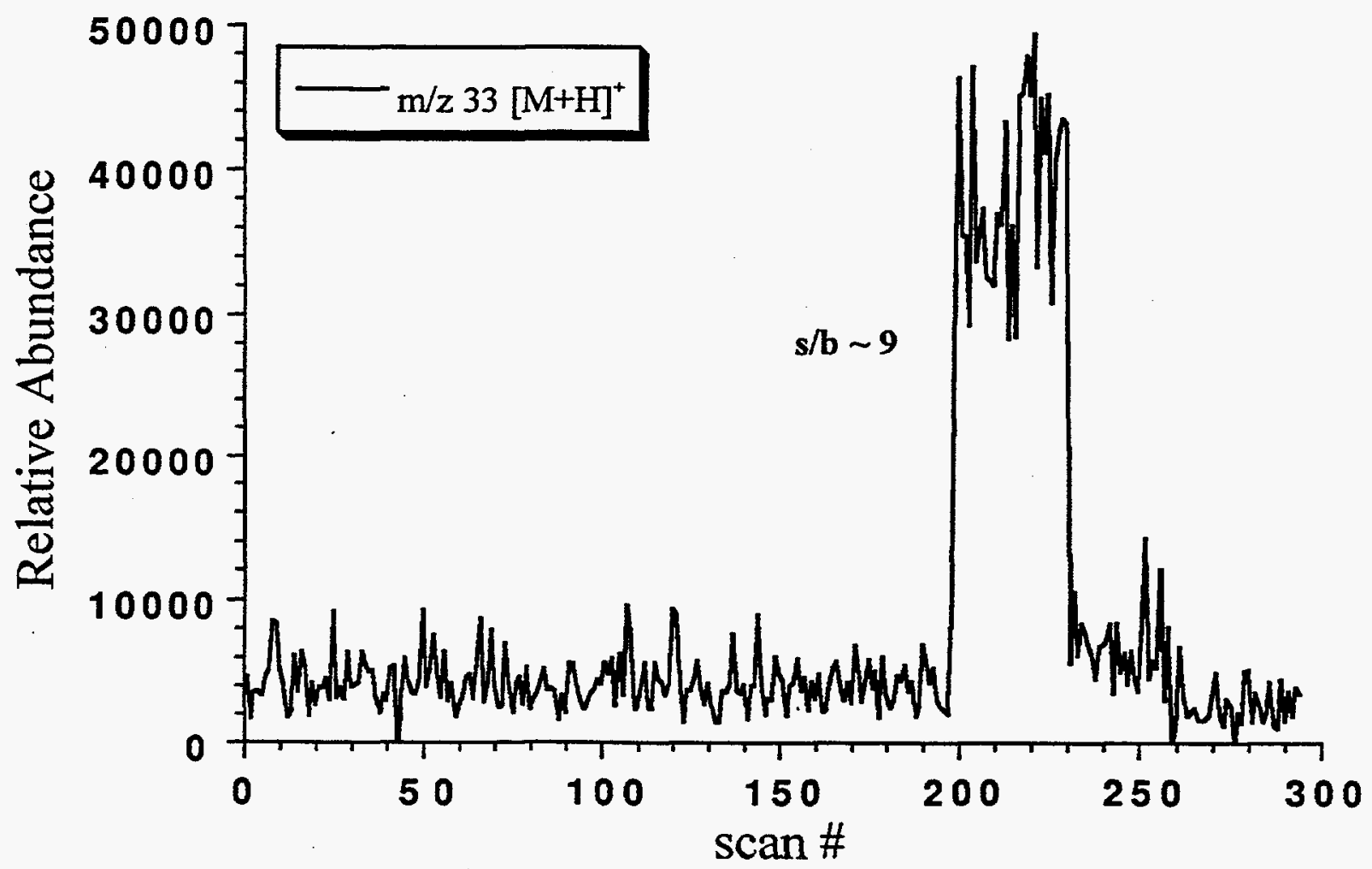

Figure 3 


\section{APPENDIX 2 - Software Development}

\section{Introduction}

Software was developed to provide a quantitative display of compound concentrations measured in engine exhaust as a function of time. This type of display is useful for correlating compound concentrations with process events. In order to display such a plot, it is necessary to convert both the scan number and $\mathrm{m} / \mathrm{z}$ abundance contained in the exhaust monitor data files to time and compound concentration, respectively. The conversion of scan number to time using the exhaust monitor data files is straightforward because the relative retention time for each scan measured from the start of a run is embedded in the DataFlex. To obtain compound concentrations, the Engine Exhaust Monitor Software extracts a mass profile for each quantitation $\mathrm{m} / \mathrm{z}$ from the DataFlex and places the data in a two-dimensional array. The next step is to apply an instrument response factor to each value in the mass profile array. The instrument response factor is compound dependent and is determined by running a series of calibration standards that cover the desired monitoring range for the engine exhaust. A user panel was designed to speed the entry of the calibration information required to generate the instrument response factors and to display the compound time vs. concentration plots. The software described here makes use of routines developed in other work at ORNL which are described elsewhere. ${ }^{1,2}$ The current software is limited to offline analysis due to the lack of access to spectral information during a run by the underlying data acquisition system. Once the underlying data system has been upgraded to support access to acquired data during a run, "realtime" viewing of compound concentrations while a test is being run will be feasible.

\section{Results and Discussion}

The user panel for the Engine Exhaust Analysis Software is shown in Fig. 1. A main command palette is found in the upper left corner of the panel. A main command summary is provided below. Below the command palette are two buttons used to scroll up and down the t vs. $C$ displays found on the right side of the panel. These buttons are only required when more than three compounds are analyzed. Below the scroll buttons are three user options that can be used to apply a dilution factor (DF), a sensitivity factor (SF) and a background subtraction (BCK). A dilution factor is useful when a exhaust sample containing high concentrations of analytes is diluted using nitrogen or other gas to reduce the likelihood of degrading ion trap performance due to space charge effects. A similar function was incorporated via the sensitivity factor although this correction factor is usually employed when the ionization time or multiplier voltage is changed from that used to generate the calibration curve. Background subtraction is a useful technique for eliminating the instrument response due to background components that also contribute to the signal of the ion(s) of interest. 
There are three $t$ vs. $C$ displays which show a plot of time vs. compound concentration, the compound name, the correlation coefficient of the calibration curve used to calculate the compound concentration and the lower and upper calibration concentrations used in generating the calibration curve. The correlation coefficients are indicative of the linearity of the calibration curve used to convert the raw abundance of ions associated with an analyte to concentration units. The low and high calibration concentrations can be compared to the high and low analyte concentrations shown on the $y$-axis of the $t$ vs. $C$ plot. If the analyte concentrations are either below the low calibration concentration or above the high calibration concentration, the user can then note that an extrapolation beyond the calibration range has been performed. Each of the $t$ vs. $C$ displays has an associated zoom and cursor control palette. These controls allow the user to blowup portions of the $t$ vs. $C$ plot and to obtain digital values by moving the cursors.

The time vs. concentration plots shown in Fig. 1 were obtained using a gas standard containing several analytes including benzene, 2-methyl-1,3-butadiene and toluene. Negative concentrations can be generated due to positive $y$-intercepts on the calibration curve used to generate the time vs. concentration plot. In some cases near background levels, the abundance being converted is smaller than the y-intercept of the calibration curve being used for the conversion, thus yielding a negative number. The best method of correcting this situation (as was done in Fig. 1) is to set the lowest displayed concentration to zero and clipping the negative numbers.

\section{Main Command Summary}

Select Files This button prompts the user for a Finnigan ITS-40 ${ }^{\mathrm{TM}}$ formatted file. The Teledyne 3DQ instrument used in the prototype engine exhaust monitor utilizes this format. Note that this software assumes that data acquisition has already taken place (i.e., offline analysis).

Plot $t$ vs. C This button actually performs the conversion of scan \# vs. abundance data to time vs. compound concentration data. A data file and a list of compounds to be quantitated (i.e., a quanlist) must have been selected prior to this option.

Save 1st Plot Save All Plots FastCali

QuanList

Spec/Profiles
This command saves the first plot in the display list to an ASCII file. This button saves all the $t$ vs. C plots to an ASCll file.

This command calls a subroutine that allows the user to create calibration curves for selected compounds. Once the calibration curves have been generated and saved to a calibration database, the subroutine returns to the Engine Exhaust Analysis Software panel.

This button is used to create a list of compounds to be used in generating the $t$ vs. C plots. The user must select compounds from a pre-existing calibration database created using the FastCali command.

This button allows the user to examine data in the selected data file by displaying mass spectra and mass profiles. 


\section{Conclusions}

The Engine Exhaust Analysis Software described here allows the user to display time vs. concentration plots for targeted compounds using data files compatible with the Finnigan ITS- $40^{\mathrm{TM}}$ specification. While this software remains an offline analysis tool, incorporation of the ability to access mass spectra as they are acquired in the underlying Teledyne 3DQ data system would make "real-time" displays feasible.

\section{Appendix 2 Figure Legend}

The main user panel of the Engine Exhaust Analysis Software showing time vs. concentration plots for benzene, 2-methyl-1,3-butadiene and toluene. The mass spectra used to generate these plots were obtained using $\mathrm{NO}^{+}$attachment and the prototype exhaust analyzer.

\section{Appendix 2 References}

1. K.J. Hart, Rapid Commun. Mass Spectrom., 1996, 10, 393-398.

2. K.J. Hart, DSITMS Analysis Software Documentation, 1996, unpublished. 


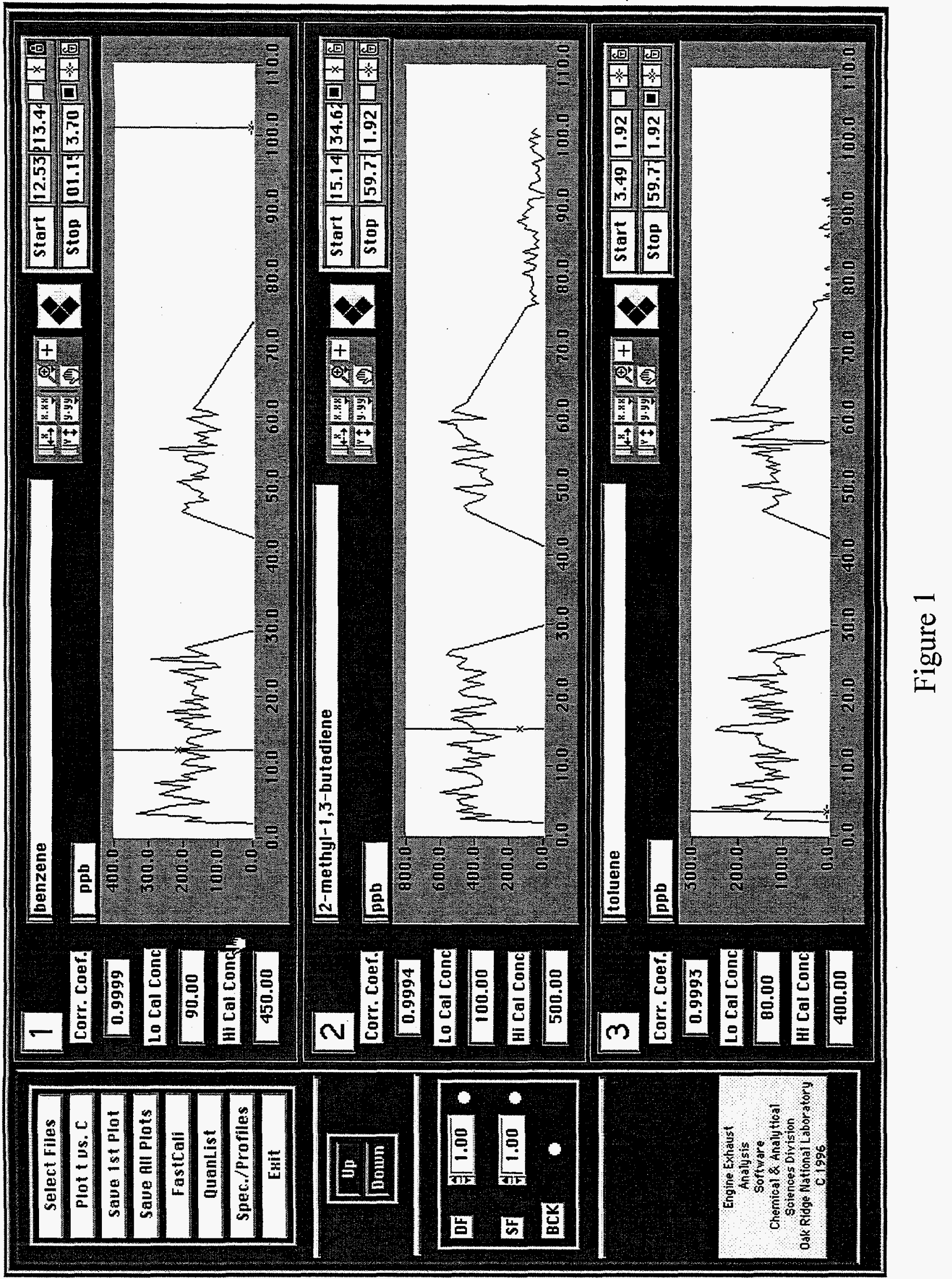




\section{APPENDIX 3 - Nitric Oxide Cl/ion Trap Sensitivity Data}

The main body of the technical report discussed signal versus concentration data for the species in the gas mix standard and, for illustration, showed plots for the ions derived from the pentenes $\left(\mathrm{M}^{+}\right)$, isopentane $(\mathrm{M}-\mathrm{H})^{+}$, and benzene $\left(\mathrm{M}+\mathrm{NO}^{+}\right)$. This appendix provides data used to select an ion accumulation period with an acceptable balance between detection limit, dynamic range, and measurement time for species in the low parts per billion to low parts per million concentration range. Plots for selected ions for which sensitivity data were acquired using an ion accumulation time of $100 \mathrm{~ms}$ are also included.

Figure $A 3 / 1$ shows a plot of the benzene $\left(M+N O^{+}\right)$ion intensity as a function of concentration for data acquired with a variety of ion accumulation periods. For ion accumulation periods of up to about $150 \mathrm{~ms}$, the increase in signal with concentration shows a roughly linear increase in signal with concentration over the entire concentration range. However, at higher ion accumulation times there is a clear leveling off of signal intensity with ion accumulation period. While lower detection limits can be obtained with higher ion accumulation periods, there is a loss in linearity at concentrations exceeding 100 $\mathrm{ppb}$ and the measurement time is extended. Therefore, an ion accumulation period of 100 ms was used for most sensitivity studies because it gives a reasonable compromise between sensitivity, dynamic range, and speed.

Figures A3/2-8 show sensitivity curves for a variety of targeted compounds shown in the format produced by the software developed as part of this project. Included in this set are ions derived from benzene, 1-butene, the pentenes, butadiene, isoprene, and the methylpentanes.

Figure A3/9 shows sensitivity data for formaldehyde. As mentioned in the main technical report, formaldehyde was not present in the gas mix standard but is of interest as an important oxygenated hydrocarbon in engine exhaust. Therefore, studies were carried out using a $5 \mathrm{ppm}$ formaldehyde in nitrogen standard. It was found that the major ion observed appeared at $\mathrm{m} / \mathrm{z} 90$, which is strong evidence that paraformaldehyde formation in the cylinder likely occurs. We therefore used the $\mathrm{m} / \mathrm{z} 90$ ion for sensitivity studies. Figure $A 3 / 9 a$ shows data acquired over the full concentration range studied ( 5 $\mathrm{ppm}$ ) and Fig. A3/9b shows the data up to $1.2 \mathrm{ppm}$. It is clear that, while there is reasonable linearity up to roughly $1 \mathrm{ppm}$, beyond about $1 \mathrm{ppm}$ there is a significant loss of linearity in signal response. 


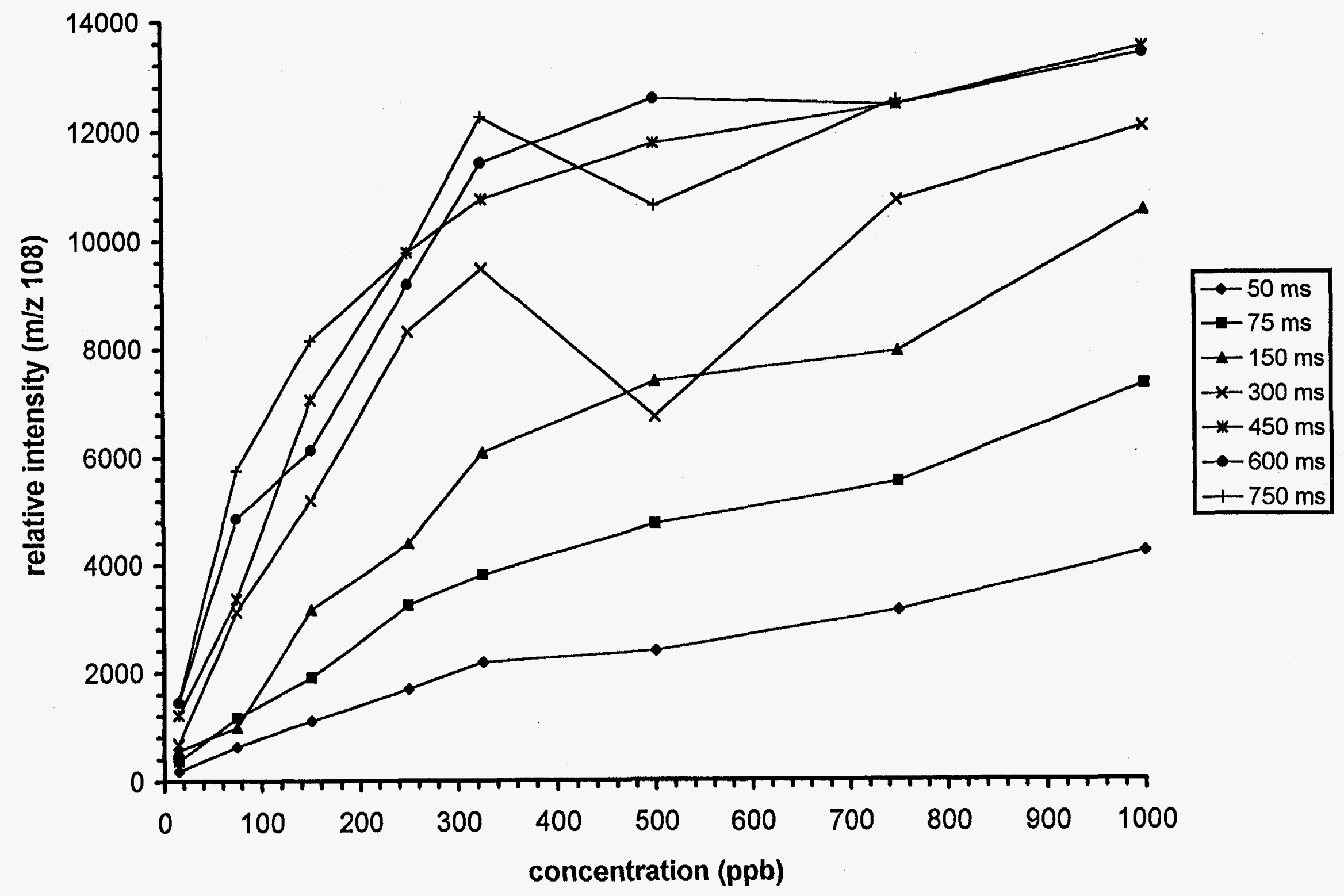

Figure 1 


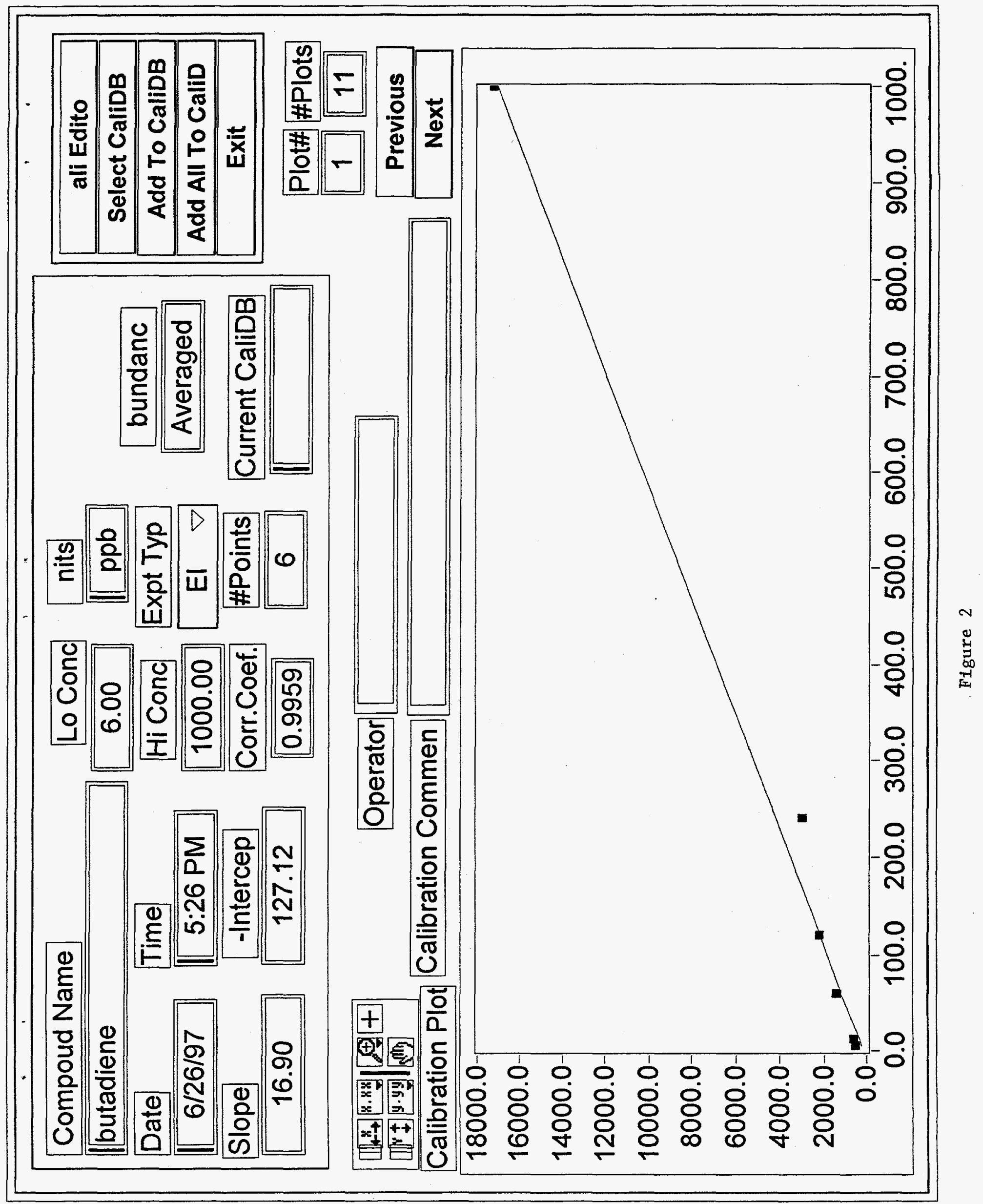




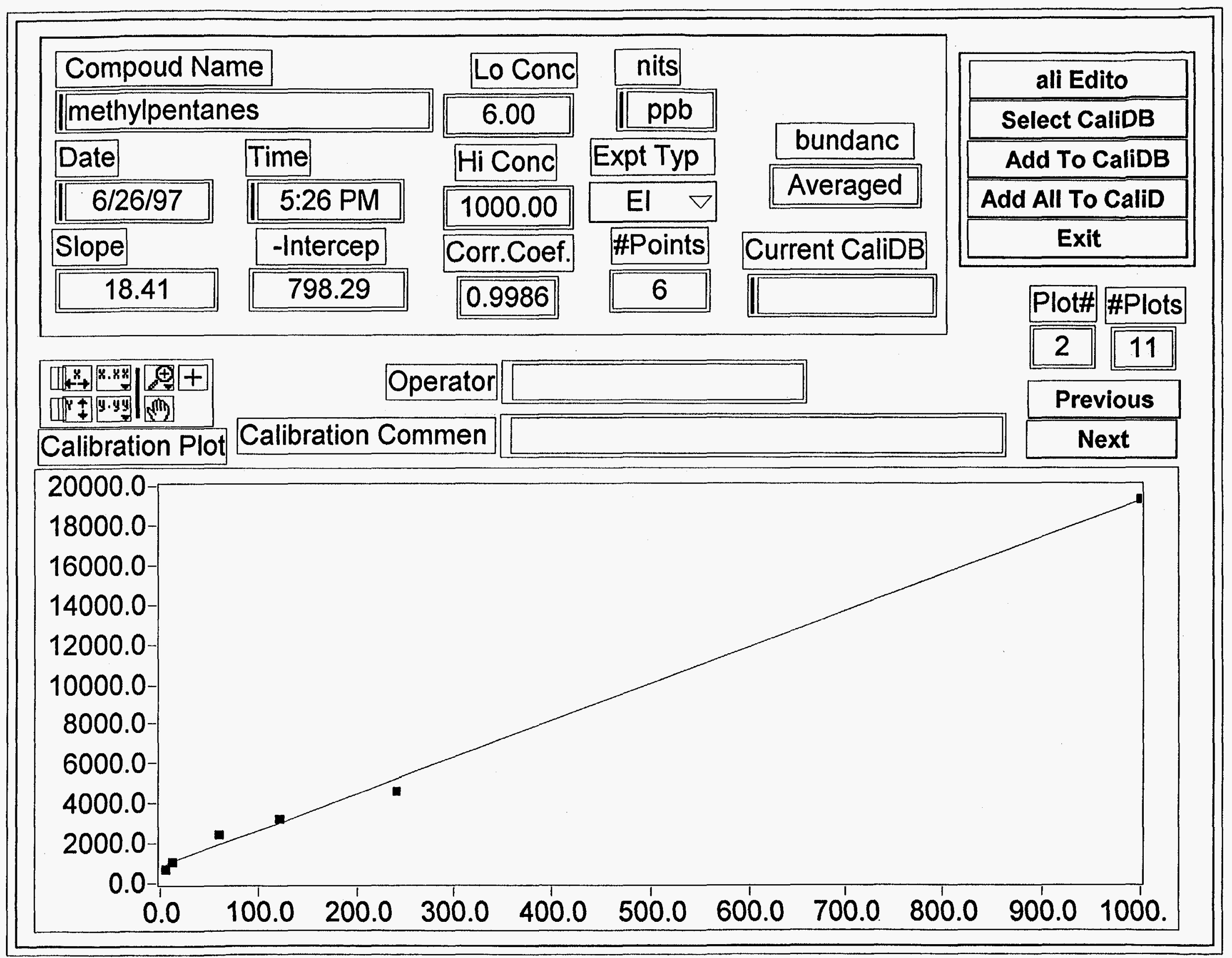




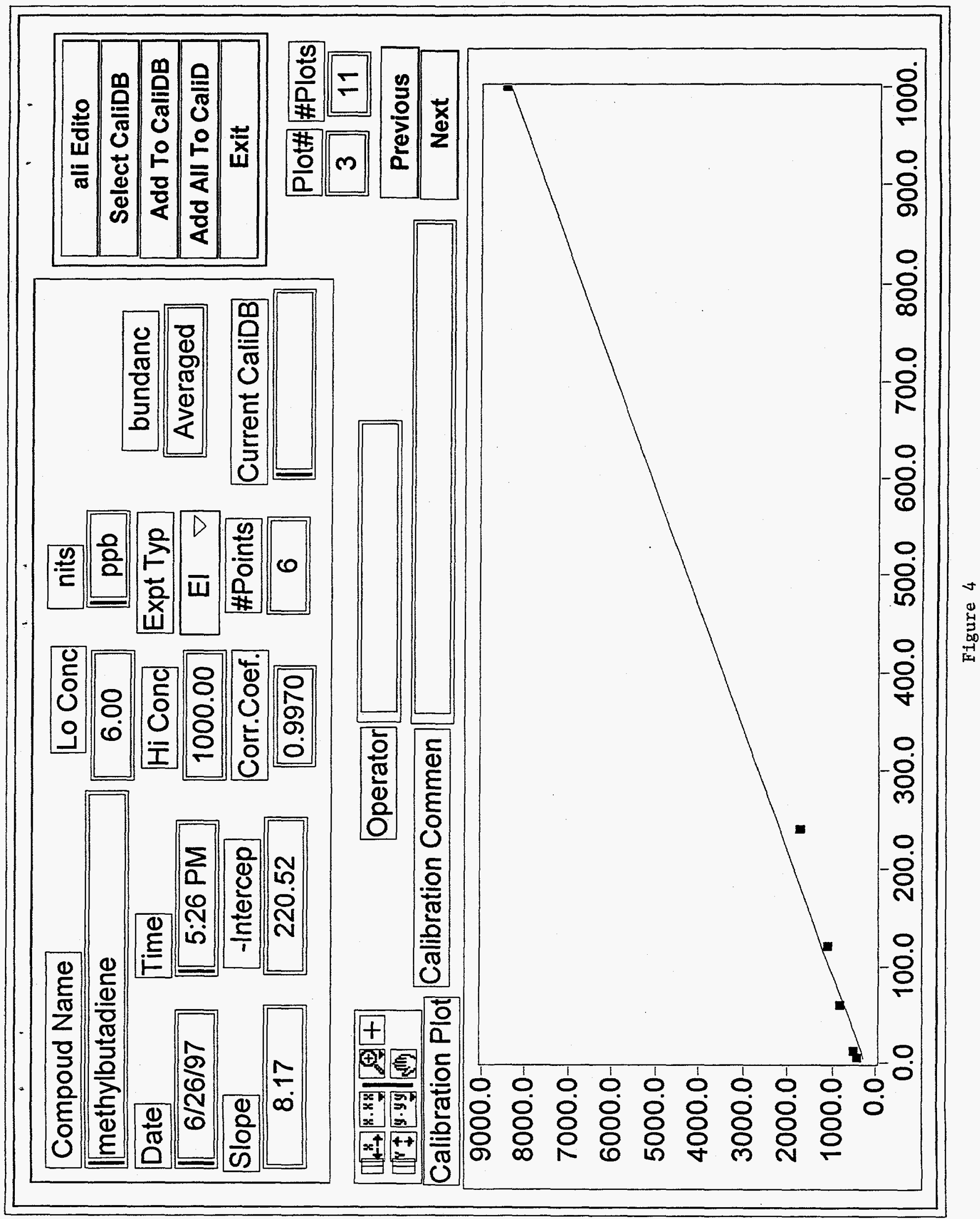




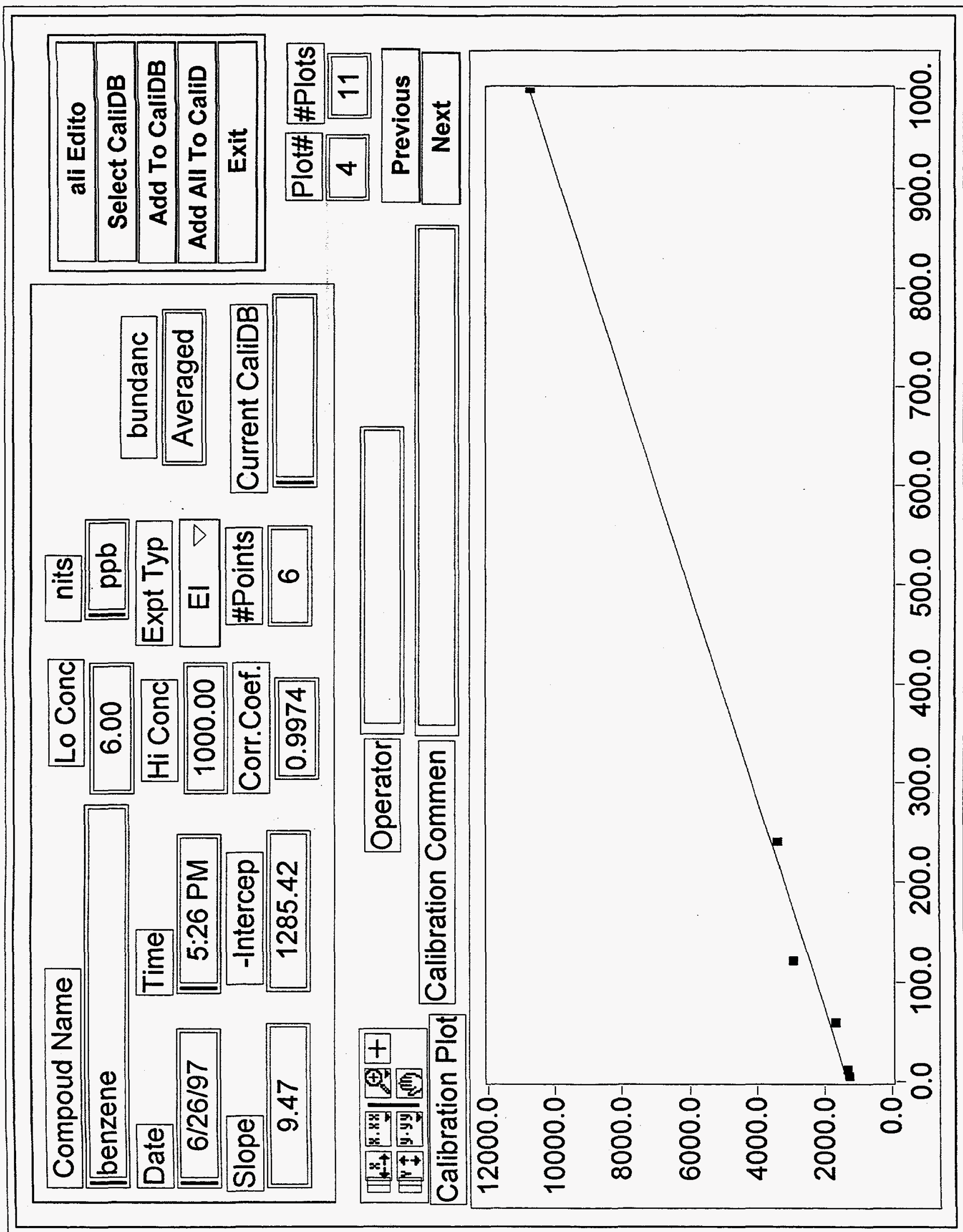




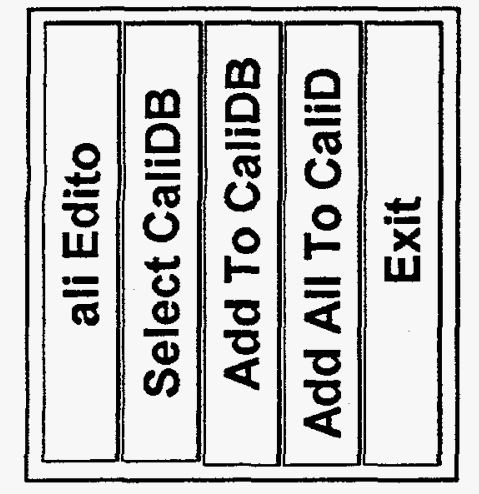

$\frac{\infty}{0} \frac{}{\mathbf{0}}+\infty$
$\frac{\not+}{0} \infty$
$\frac{\infty}{\infty}$

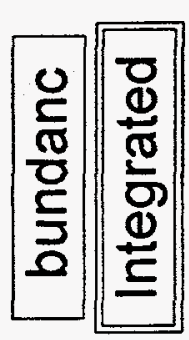

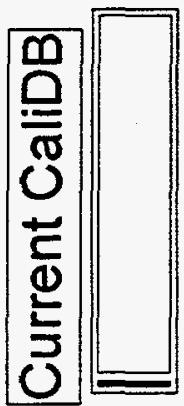

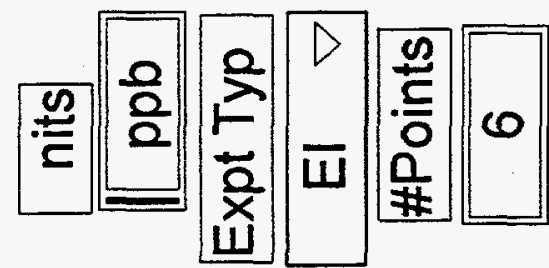

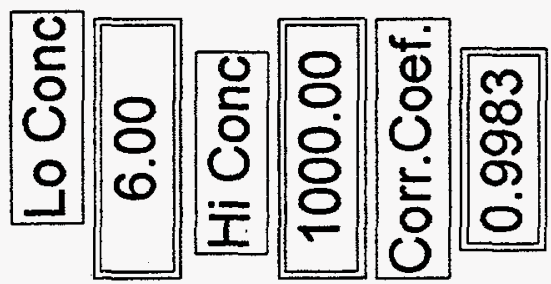
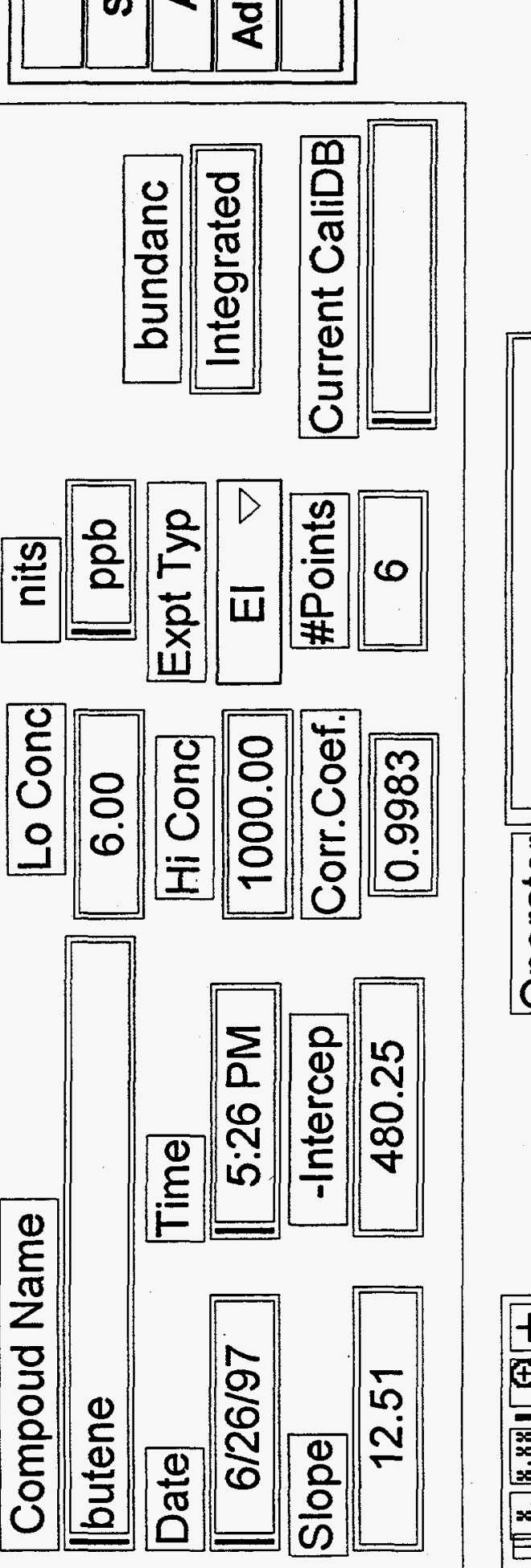
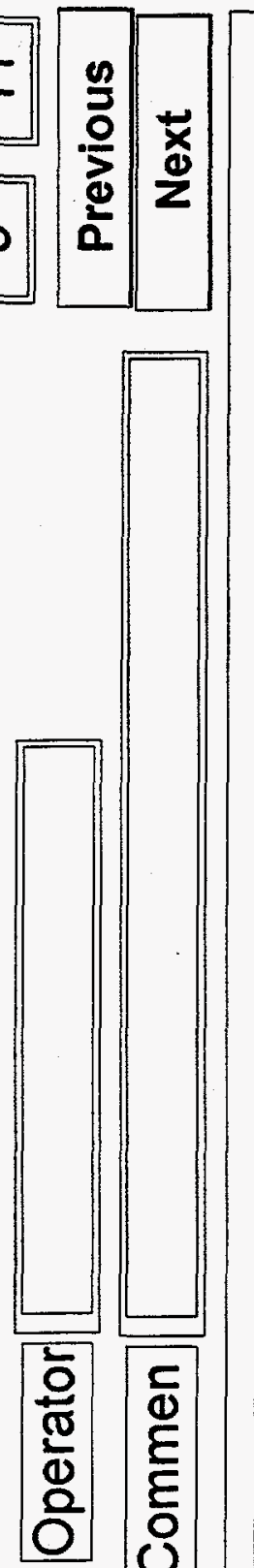


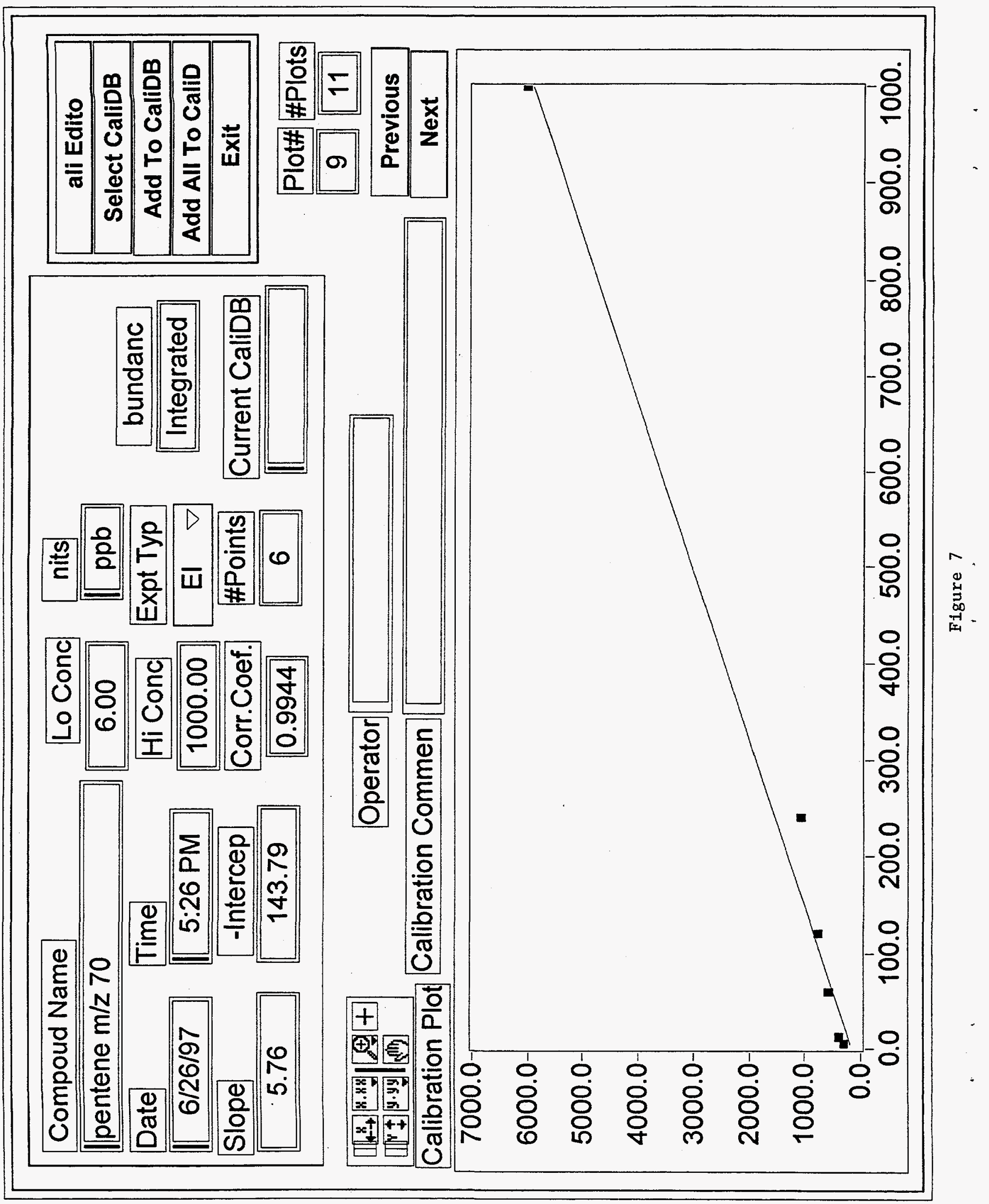




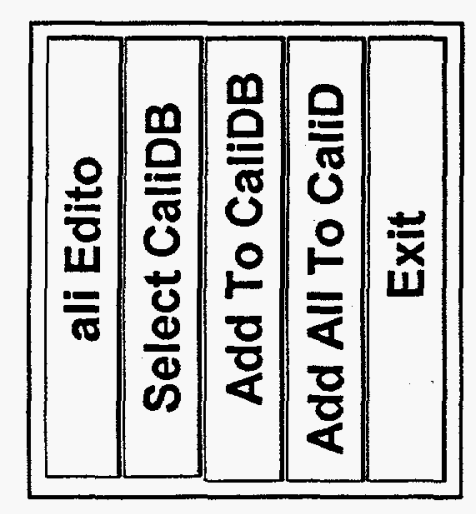

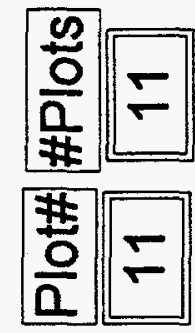

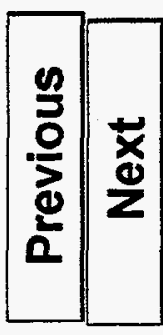

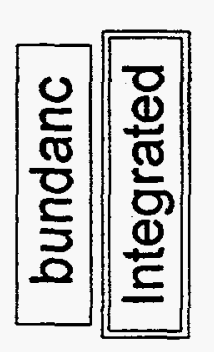

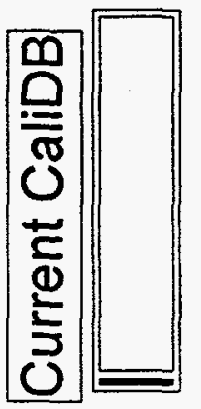

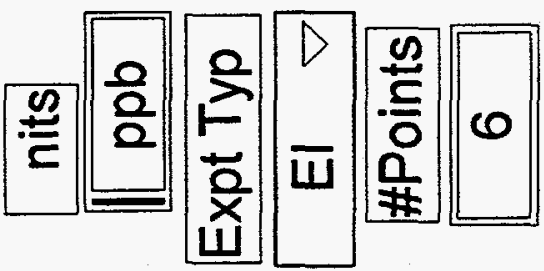

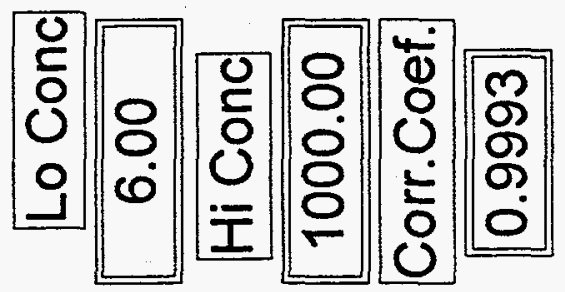

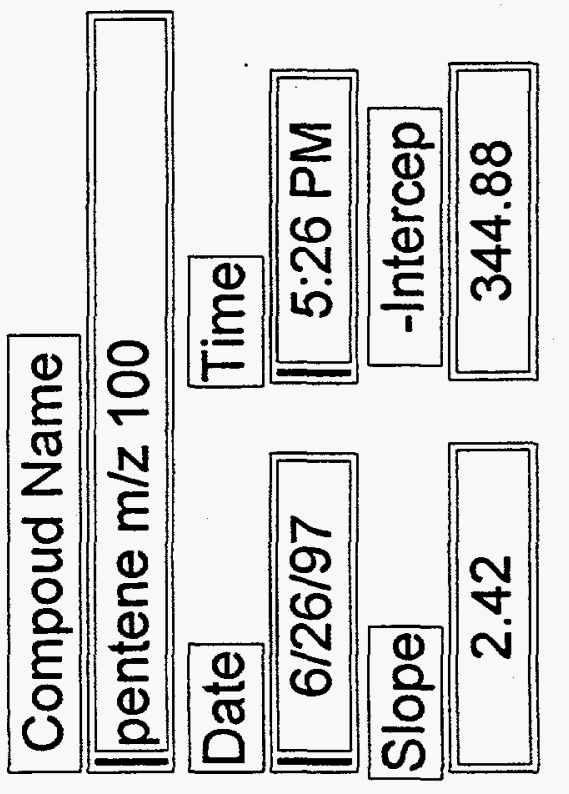

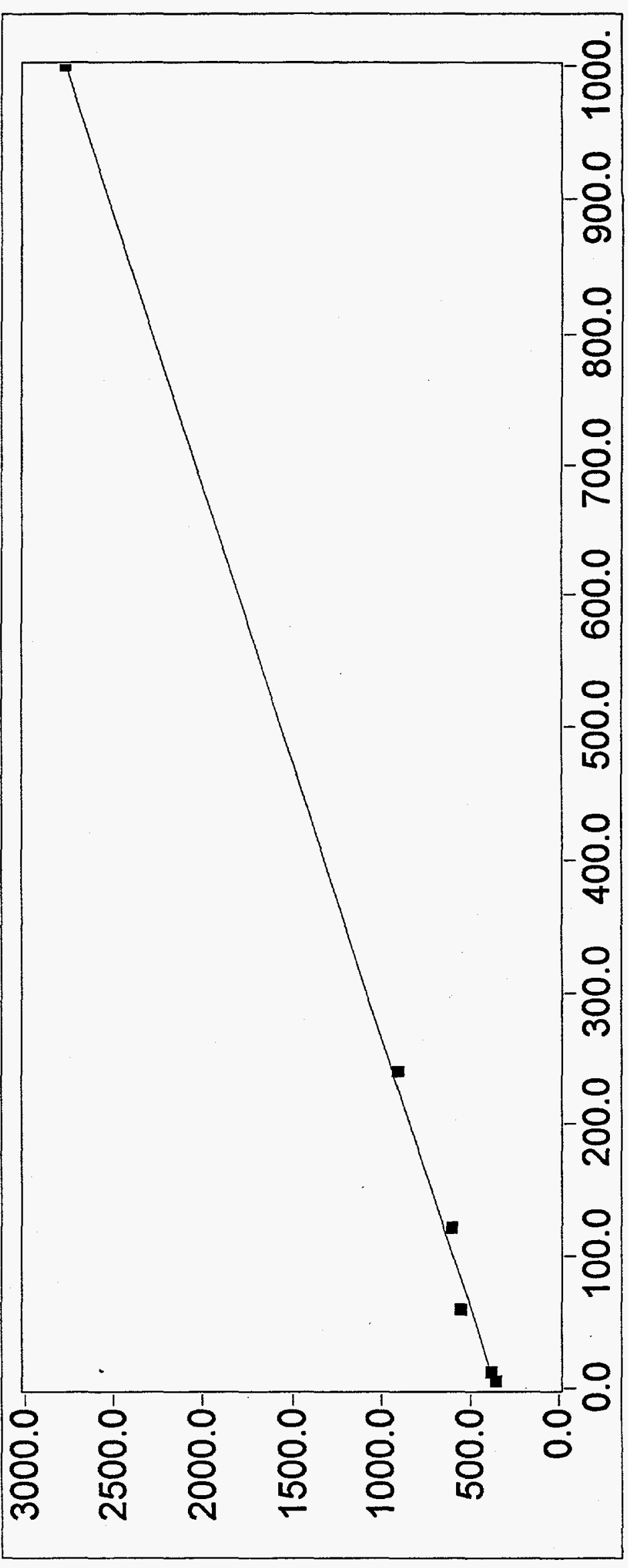



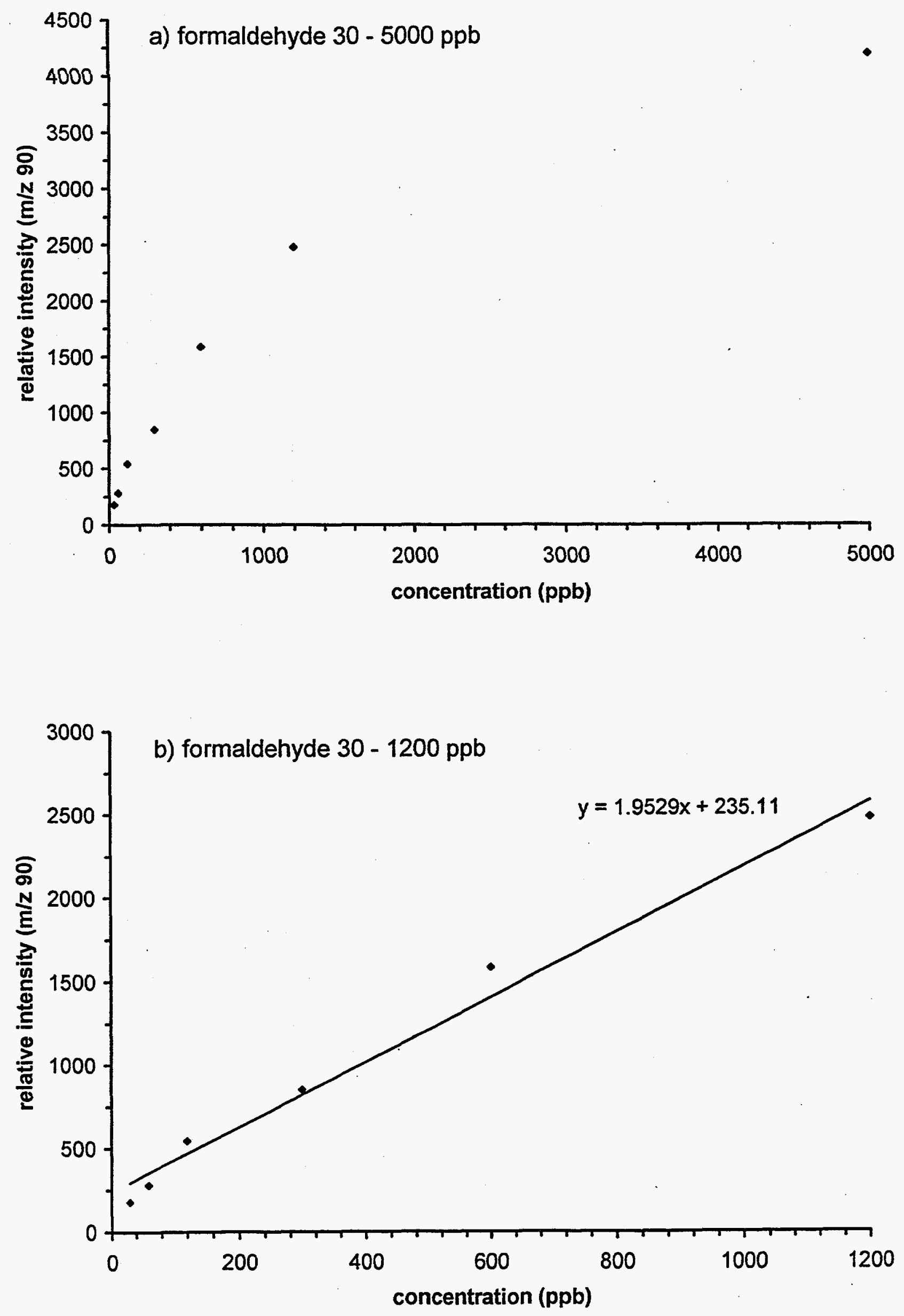


\section{APPENDIX 4 - Tandem Mass Spectra}

Table 3 of the main technical report summarizes the main parent ion/product ion information obtained from the nitric oxide Cl/ion trap study of the gas mix standard. Figures A4/1-8 give tandem mass spectrometry results for butadiene, the pentenes, isopentane, the methylpentanes, isoprene, benzene, toluene, and the $\mathrm{C}_{2}$-benzenes. The data show both pre-collisional activation and post-collisional activation information so that the approximate MS/MS efficiency can be determined. 
Profile Spectrum Plot

Data File: C:ITELEDYNE3DQWATALOCT2SL.MS

Sample: ASGDI-pilsed;butene $\square$

Scan Range: $70-90$

Max Intensity: 541.57

Points: 2123

Total Intensity: $\mathbf{5 2 , 8 6 2 . 9 0}$

Plot Range: 1 - 875

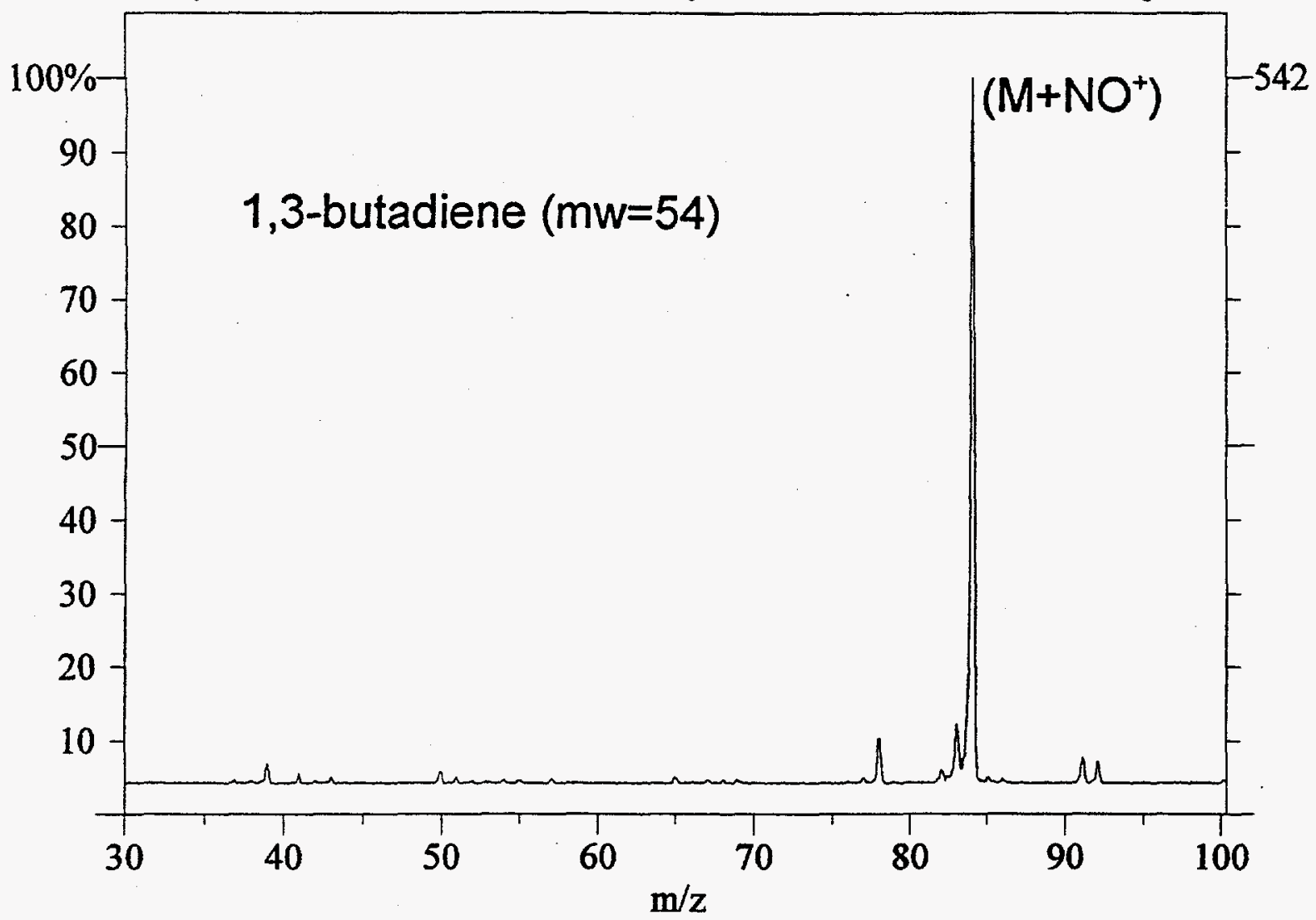

Profile Spectrum Plot

Data File: C:ITELEDYNEUDQDATAlOCT25I.MS

Sample: ASGDI-pilsed;butene $D$

Scan Range: $10-30$

Date: Oct-25-1995

Time: 10:24:00 am

Max Intensity: 496.48

Total Intensity: $\mathbf{5 2 , 8 7 0 . 8 1}$

Points: 2123

Plot Range: 1 - 873

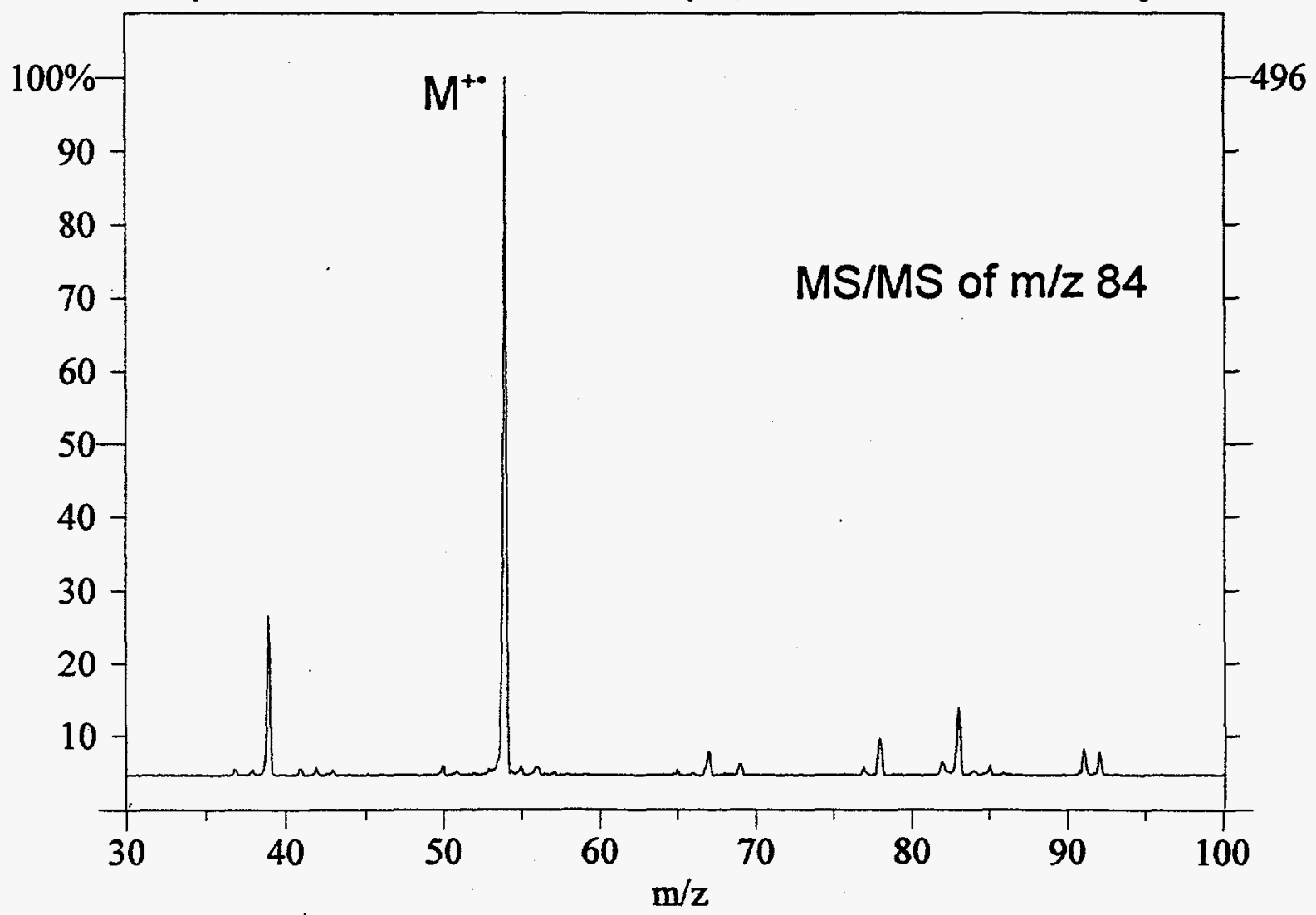


Profile Spectrum Plot

Data File: CATELEDYNEI3DQWDATAlOCT2SP.MS

Sampie: ASGDI-pulsed; m/z700

Scan Range: 95 - 105

Max Intensity: 14,874.73

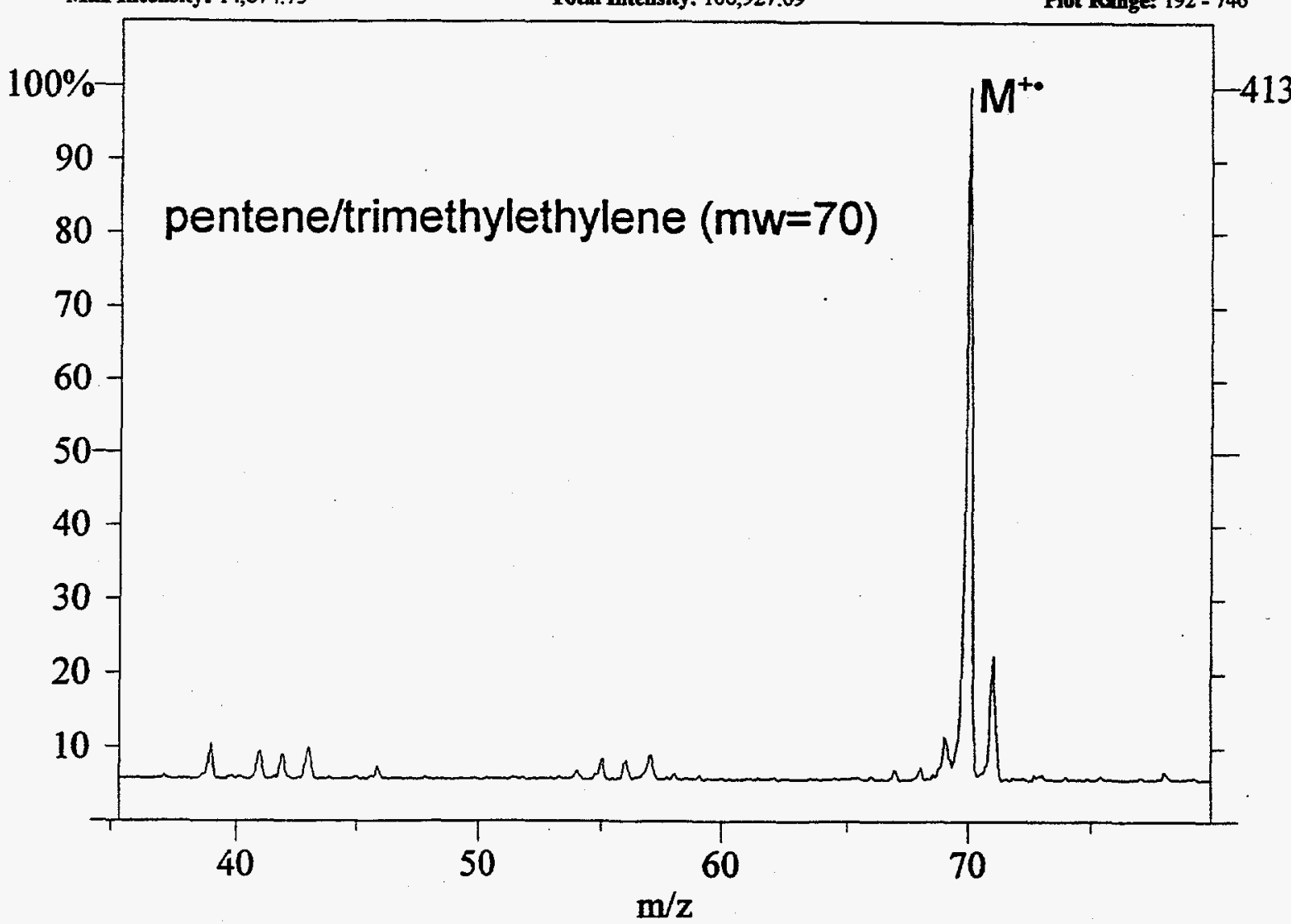

Date: Oct-25-1995

Points: 2248

Total Intensity: $106,927.09$
Time: 11:21:00 am

Profile Spectrum Plot

Data File: C:ITELEDYNEI3DQLDATAIOCT2SP.MS

Sample: ASGDI-pulsed; $m / z 700$

Scan Range: $30-40$

Max Intensity: 14,785.64

Total Intensity: $106,969.91$

Date: Oct-25-1995

Time: 11:21:00 am

Points: 2248

Plot Range: 192 - 746

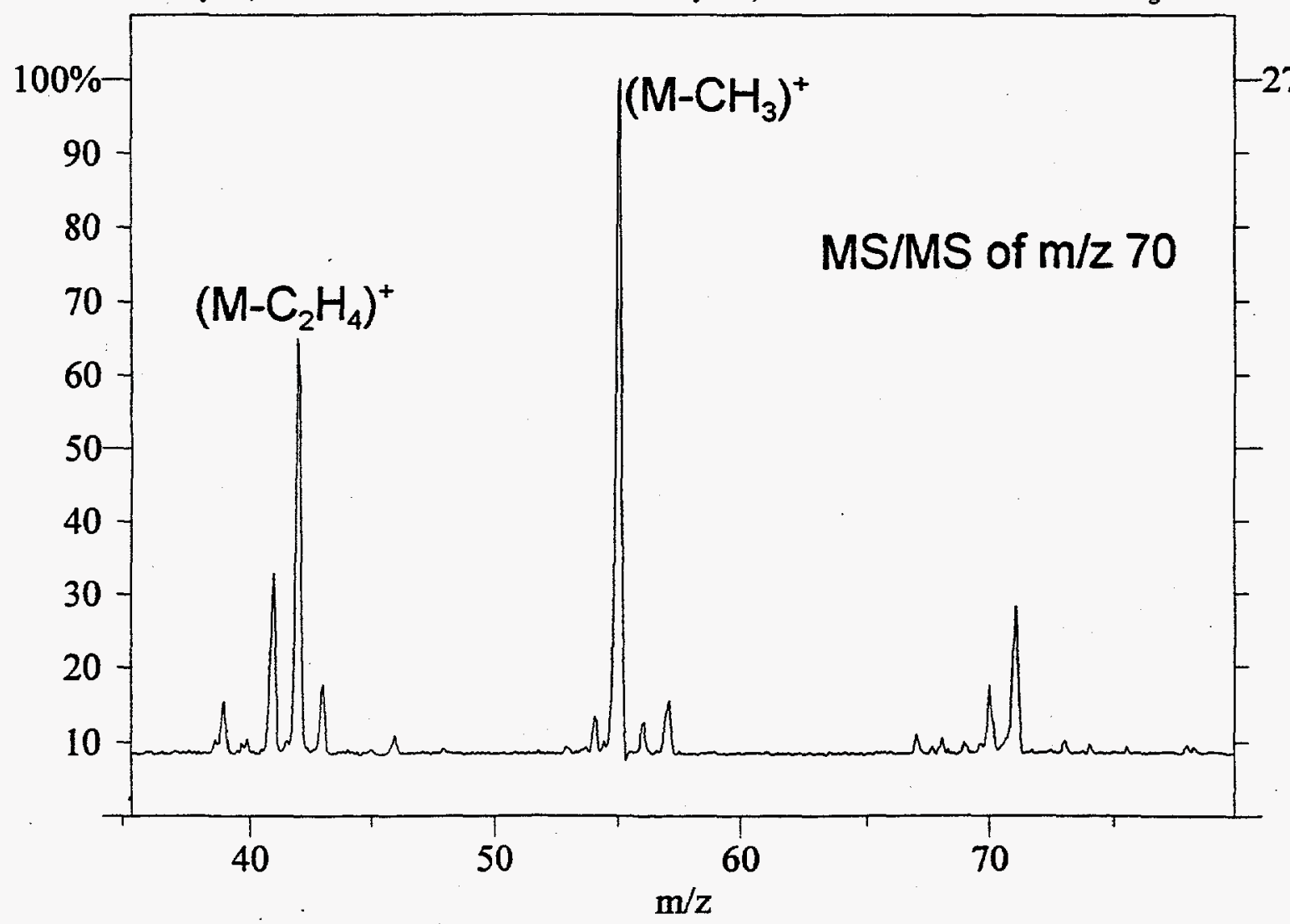


Profile Spectrum Plot

Data File: C:ITELEDYNEI3DQDATAIOCT25MMS

Sample: ASGDI-pilsed; isopentane $]$

Scan Range: $70-80$

Max Intensity: $14,631.27$

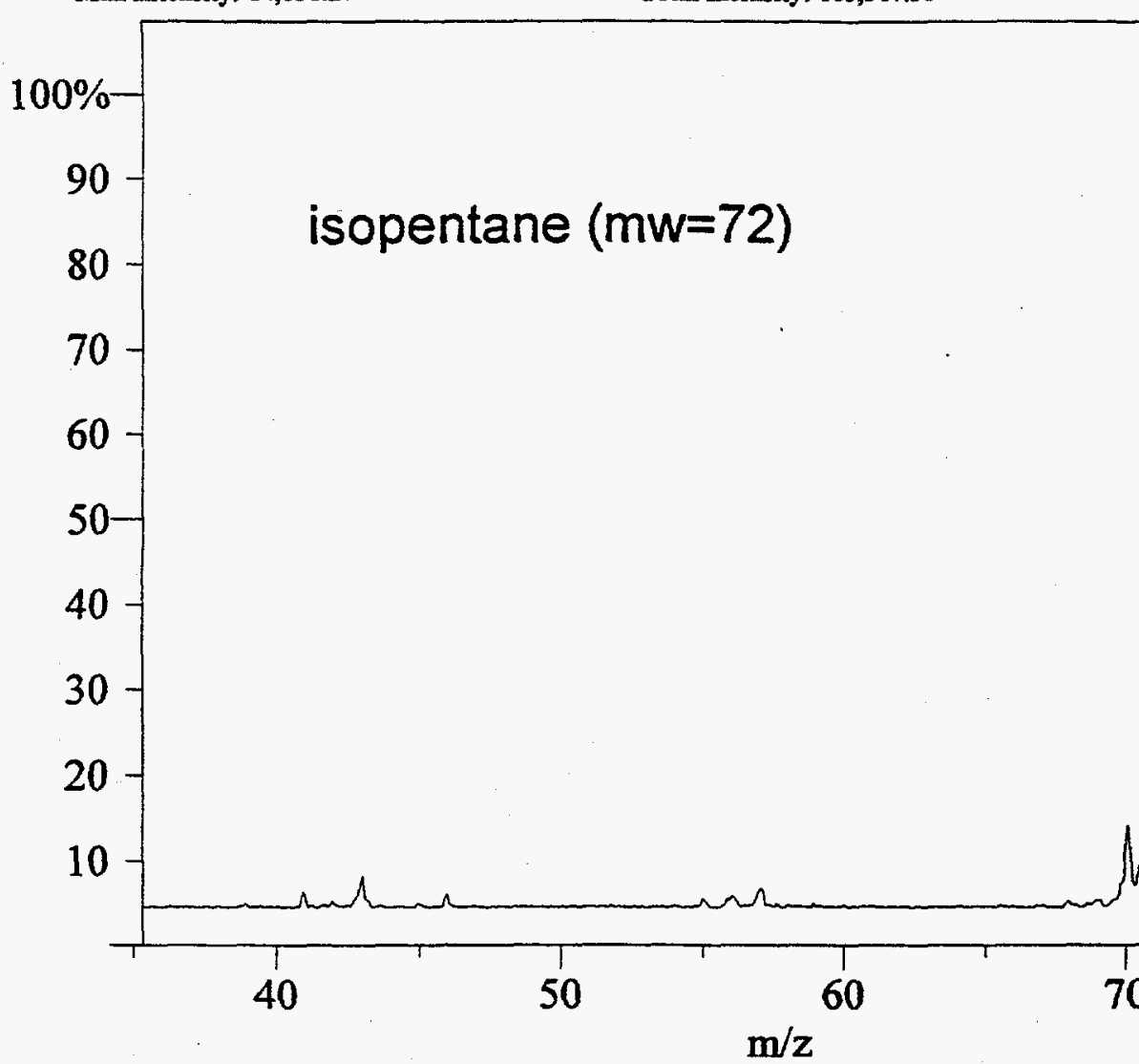

Date: Oet-25-1995

Time: 11:12:00 am

Points: 2248

Plot Range: 192 - 748

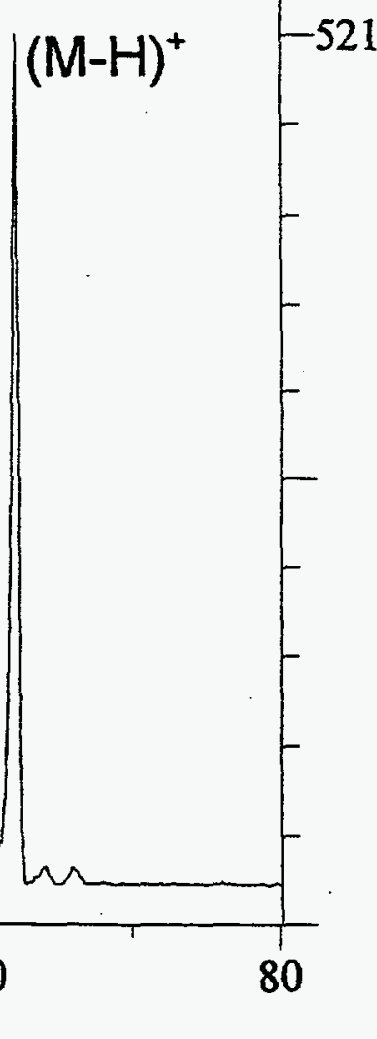

Profile Spectrum Plot

Data File: C:ITELEDYNEL3DQWATAIOCT25M.MS

Sample: ASGDI-pilsed; isopentaneD

Scan Range: 5- 15

Max Intensity: 14,100.18

Total Intensity: $103,876.64$

Date: Oct-25-1995

Time: 11:12:00 am

Points: 2248

Plot Range: $189-748$

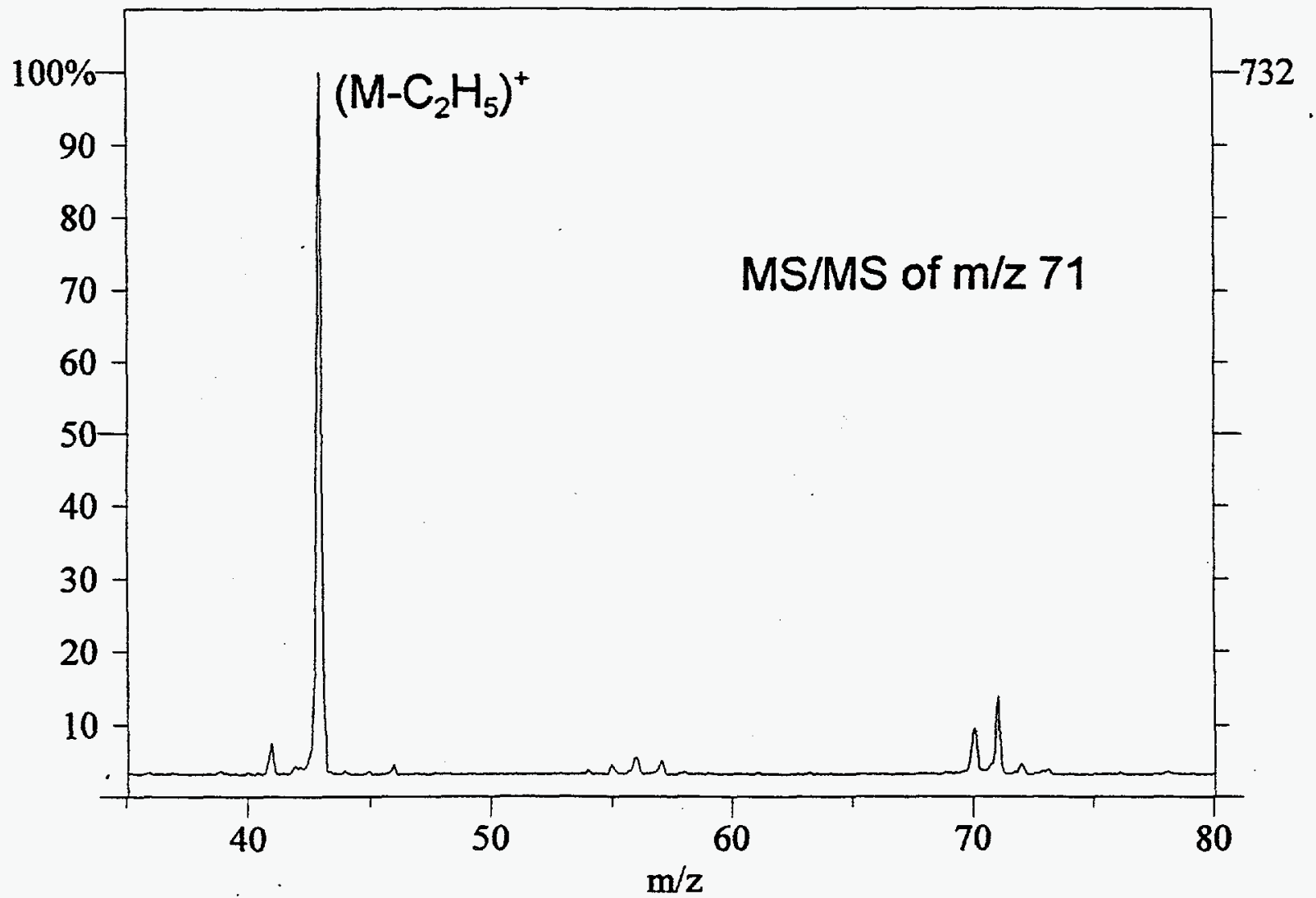


Profile Spectrum Plot

Data File: C.ITELEDYNEL3DQLDATALCT25J.MS

Sample: ASGDI-pilsed; hexanes

Scan Range: 52-62

Max Intensity: 629.64
Date: Oct-25-1995

Time: 10:30:00 am

Points: 2123

Piot Range: 1 - 873

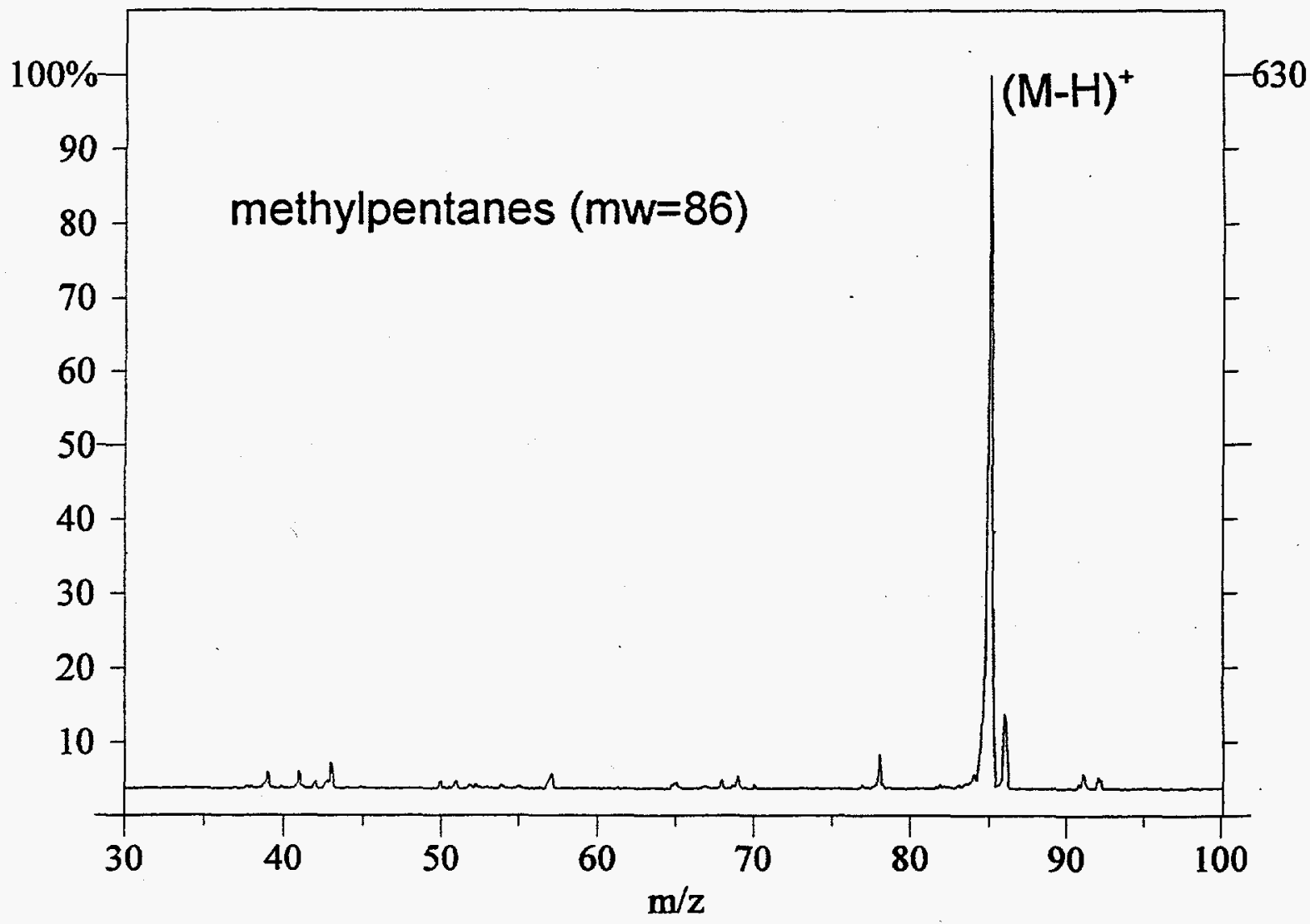

Profile Spectrum Plot

Data File: C:ITELEDYNEL3DQLDATAIOCT25J.MS

Sample: ASGDI-pilsed; hexanes

Scan Range: 5 - 15

Date: Oet-25-1995

Max Intensity: 671.82

Total Intensity: $54,610.36$

Time: 10:30:00 am

Points: 2123

Plot Range: 1 - 873

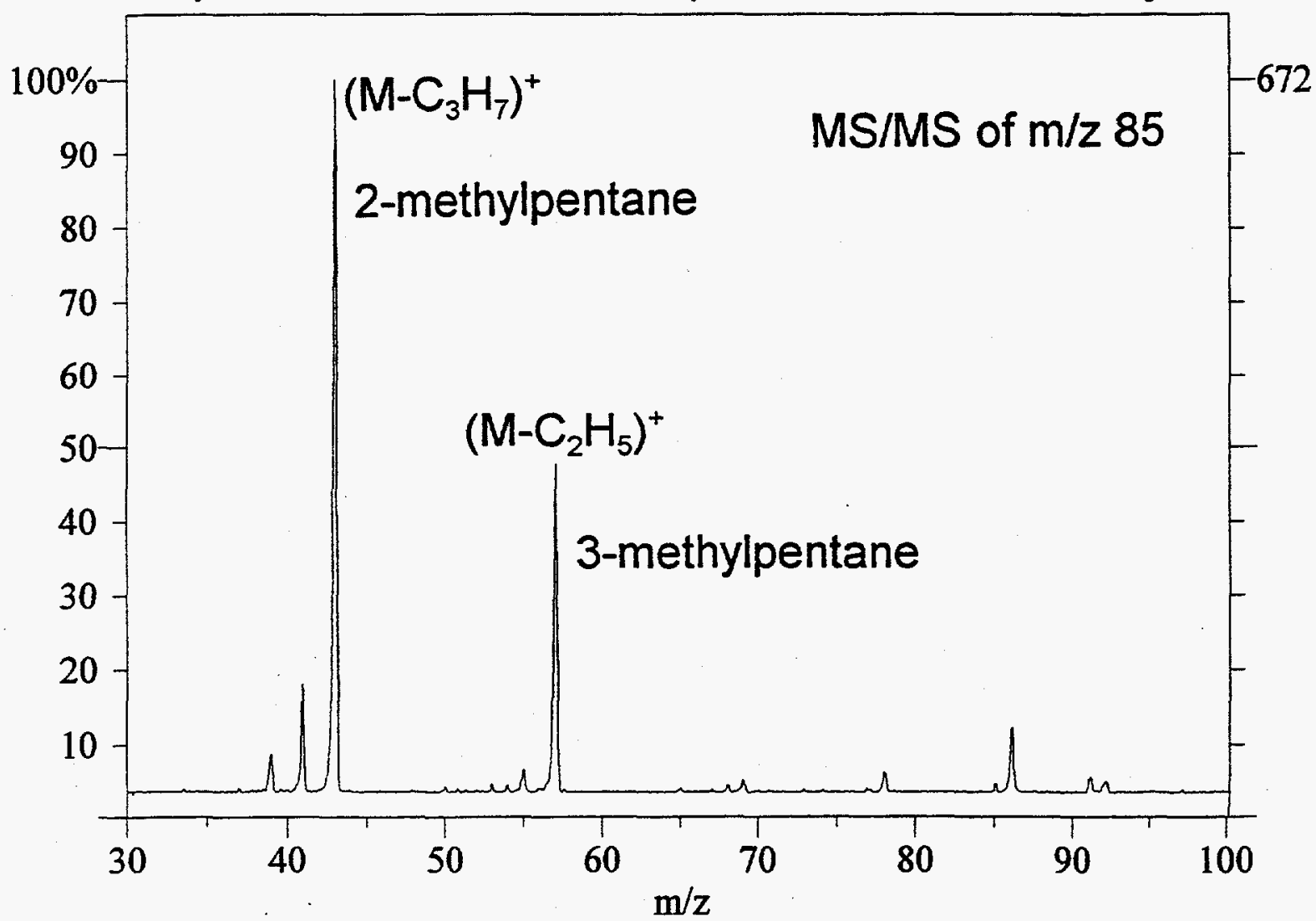


Sample: ASGDI-pilsed; 2-methyl-1,3-butadiene]

Scan Range: 10 - 20

Max Intensity: 463.55

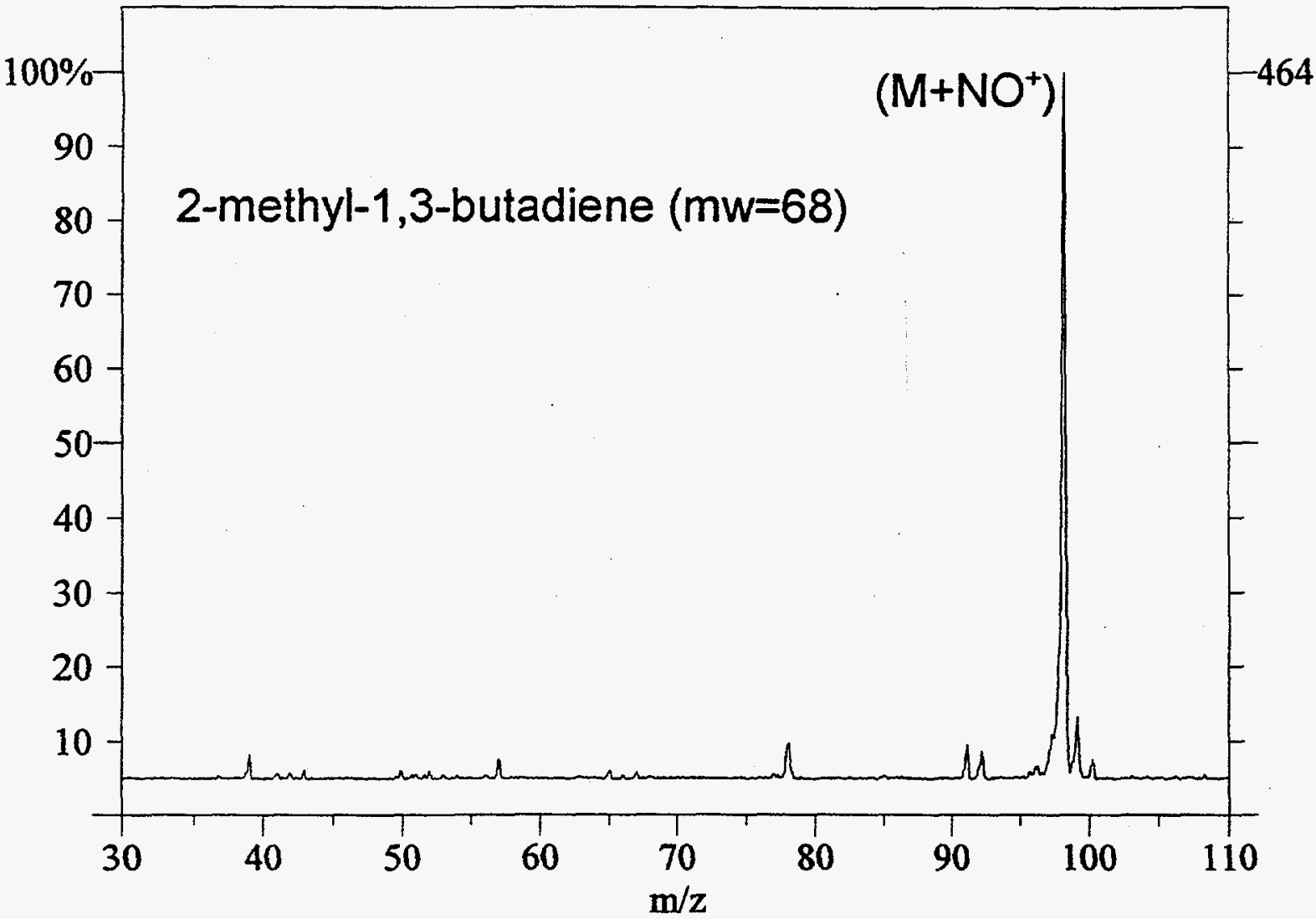

Prolle Spectrum Plot

Sample: ASGDI-pilsed; 2-methyl-1,3-butadiene]

Scan Range: $50-60$

Max Intensity: 443.36

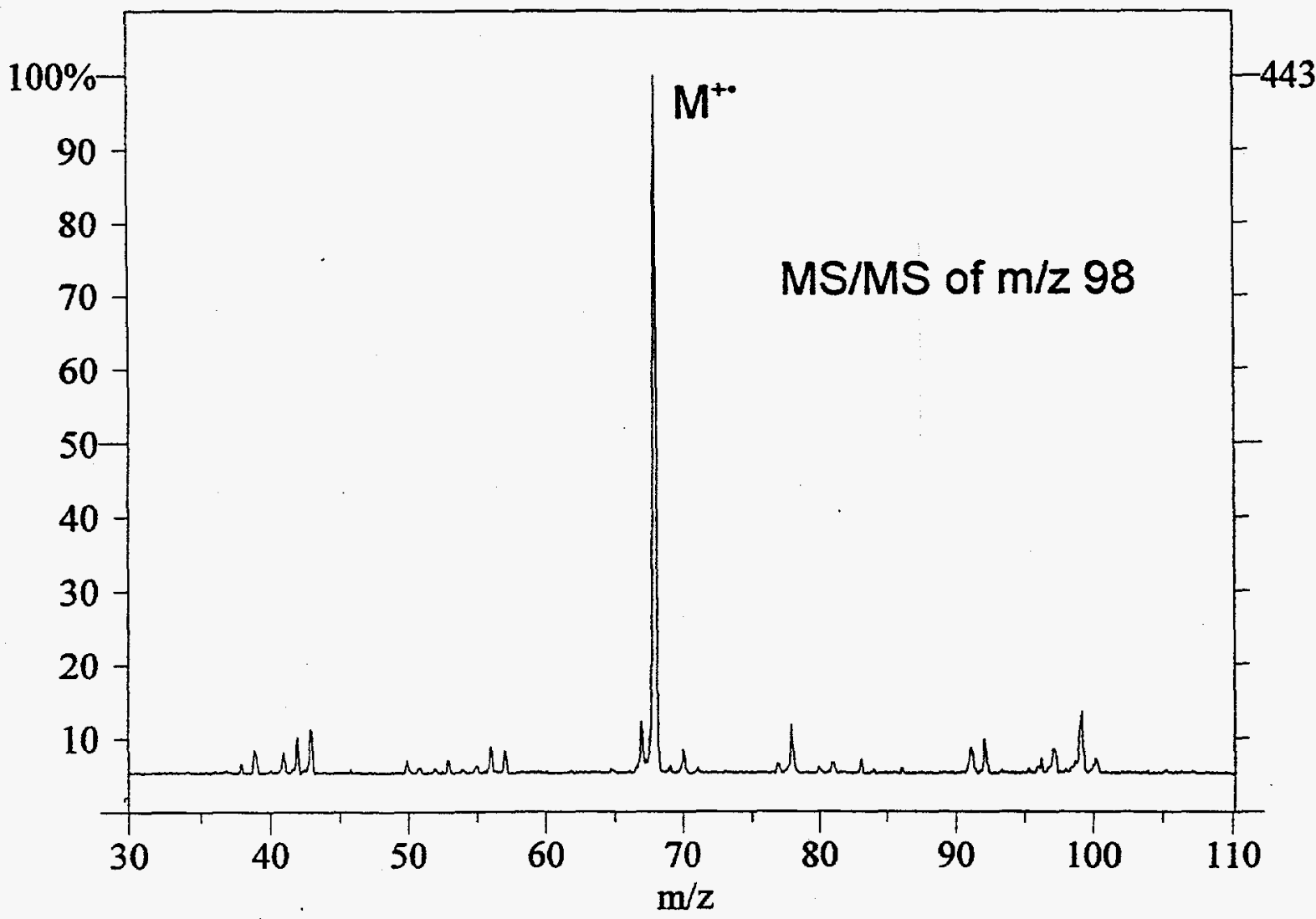


Profile Spectrum Plot

Data File: C:ITELEDYNEL3DQWATAIOCT25RMS

Sample: ASGDI-pulsed; (benzene+NO) $]$

Scan Range: 55-65

Max Intensity: 332.64

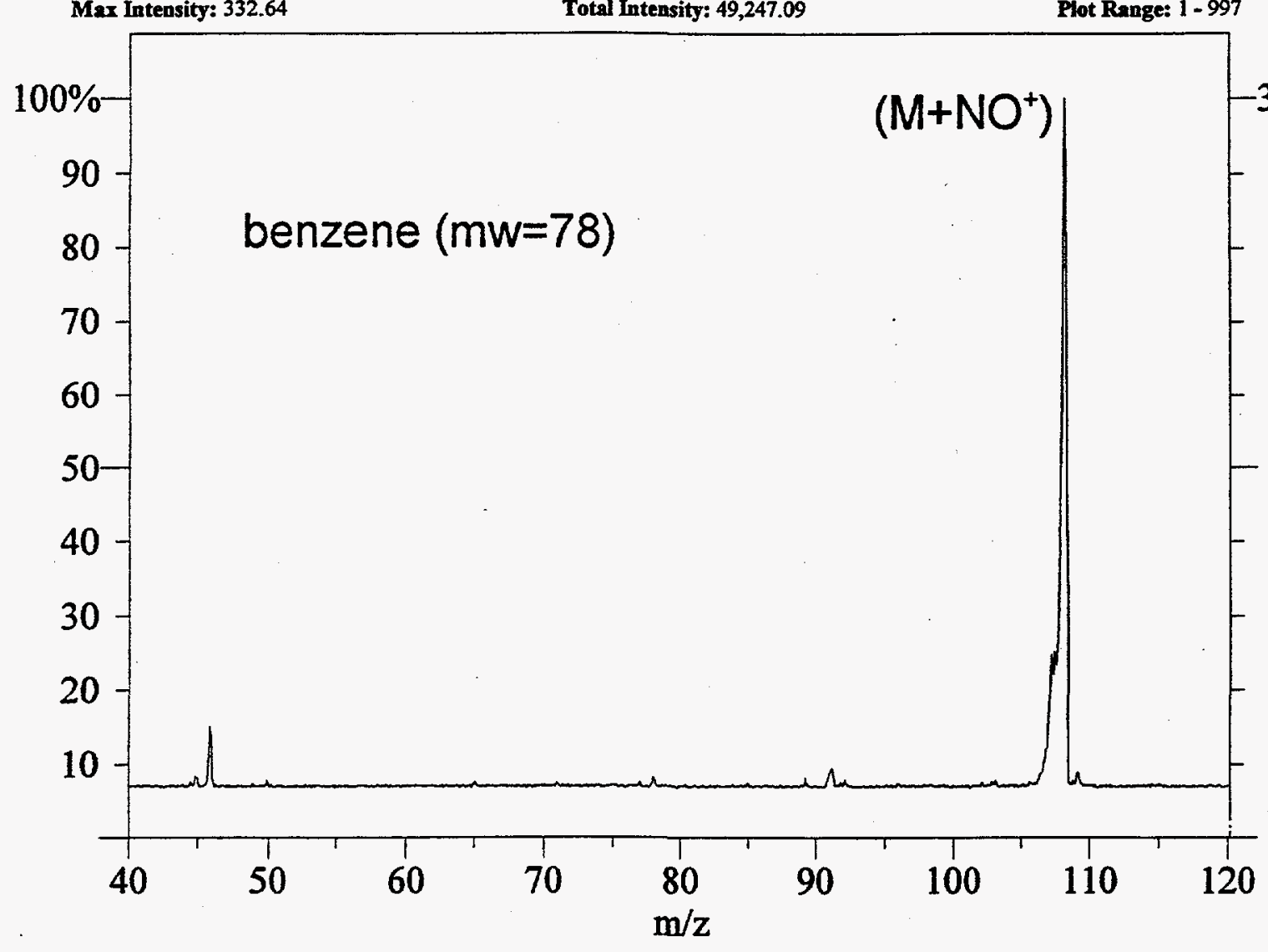

Time: 12:10:00 pm

Points: 1998

Plot Range: 1 - 997
Date: Oct-25-1995
Profile Spectram Plot Data File: C:ITELEDYNE3DQDATAIOCT25R.MS Sample: ASGDI-puised; (benzene+NO) $\square$

Scan Range: 15-25

Max Intensity: 321.64

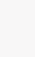

Total Intensity: $48,290.00$

Date: Oct-25-1995

Time: 12:10:00 pm

Points: 1998

Plot Range: 1 - 997

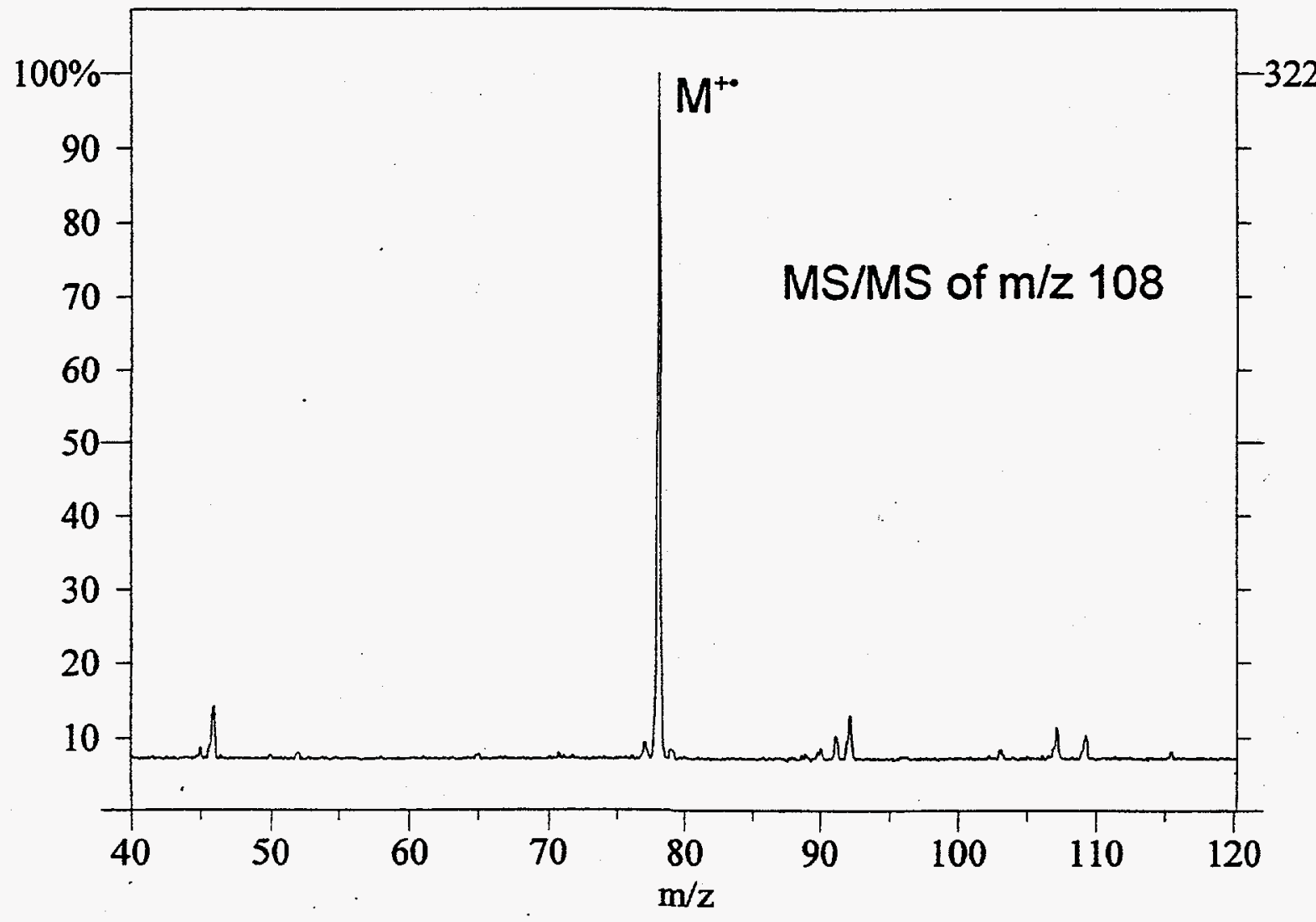


Profile Spectrum Plot

Data File: C:ITELEDYNEUDQDATAIOCT25C.MS

Sample: ASGDI-pilsed;toluene+NOD

Scan Range: 15 - 30

Max Intensity: 210.81

Points: 1998 Plot Range: 1 - 1184

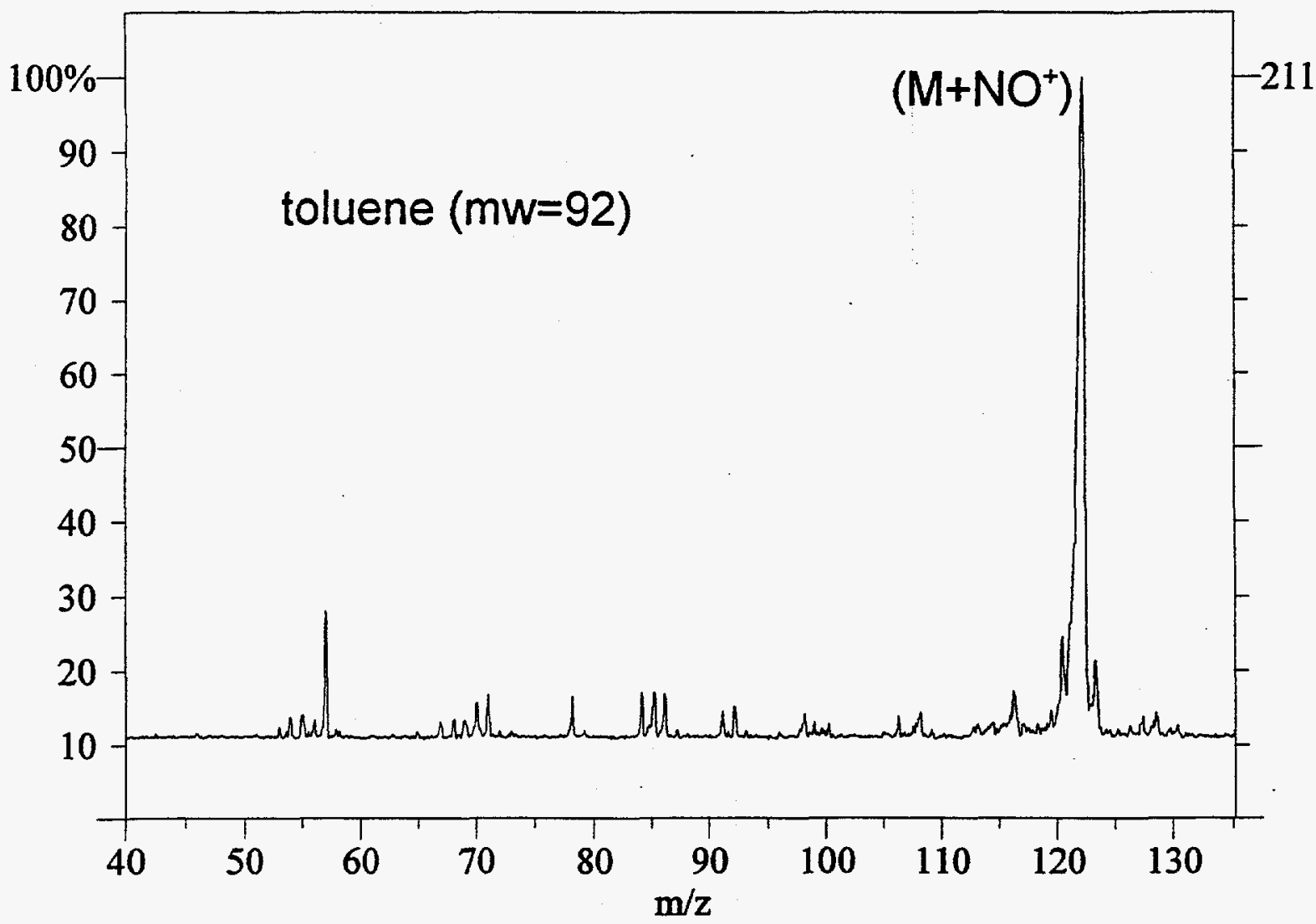

Profile Spectrum Plot

Data File: C:ITELEDYNEI3DQLDATAIOCT25C.MS

Sample: ASGDI-pilsed;toluene+NOU

Scan Range: 62 - 72

Max Intensity: 232.18

Total Interisity: $48,604.45$

Date: Oct-25-1995

Time: 07:38:00 am

Points: 1998

Plot Range: 1 - 1184

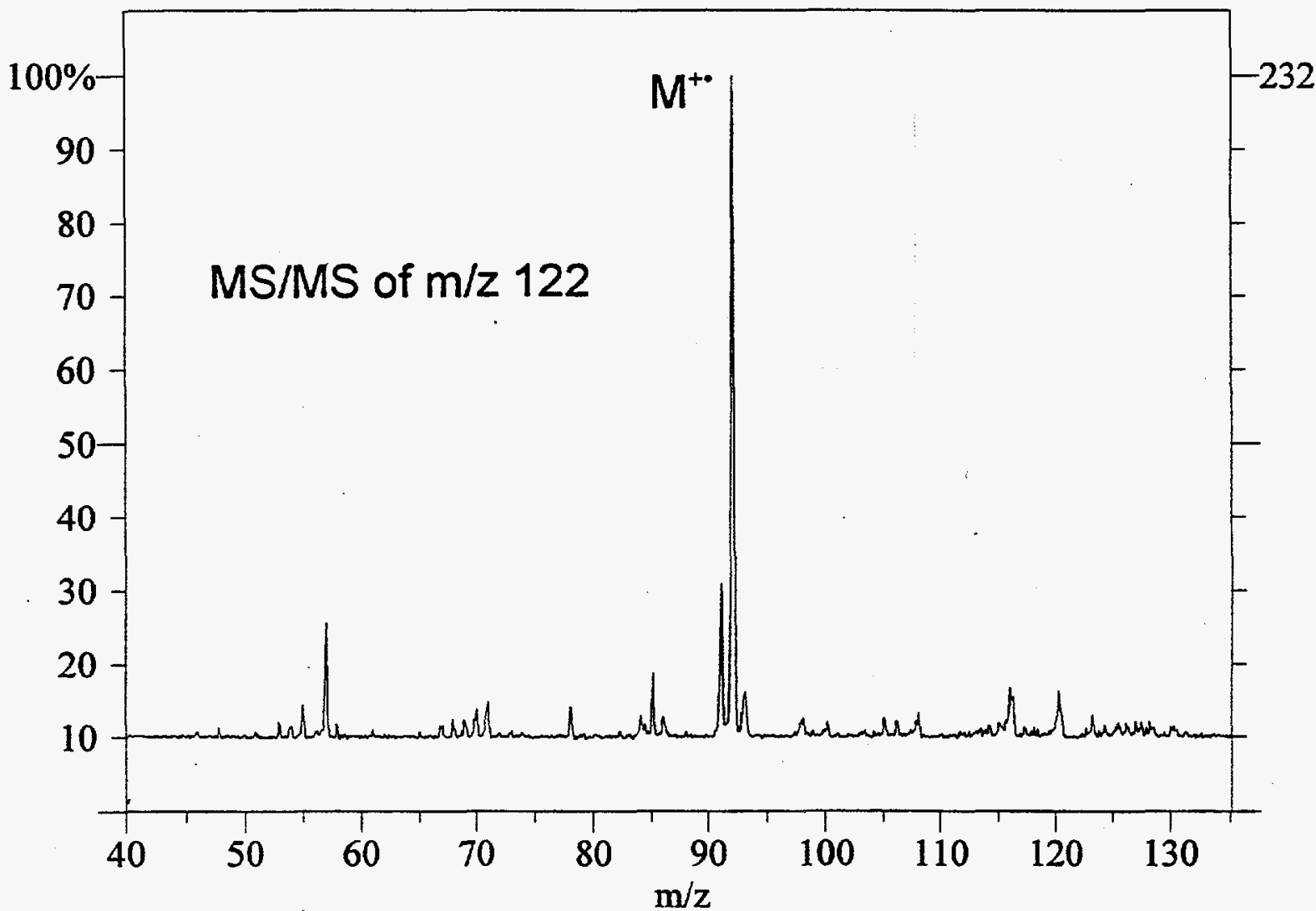


Profile Spectrum Plot

Data File: C:ITELEDYNEl3DQDATAIOCT25D.MS

Sample: ASGDI-pilsed;C2 benzenes+NOD

Scan Range: $10-30$

Max Intensity: 181.00

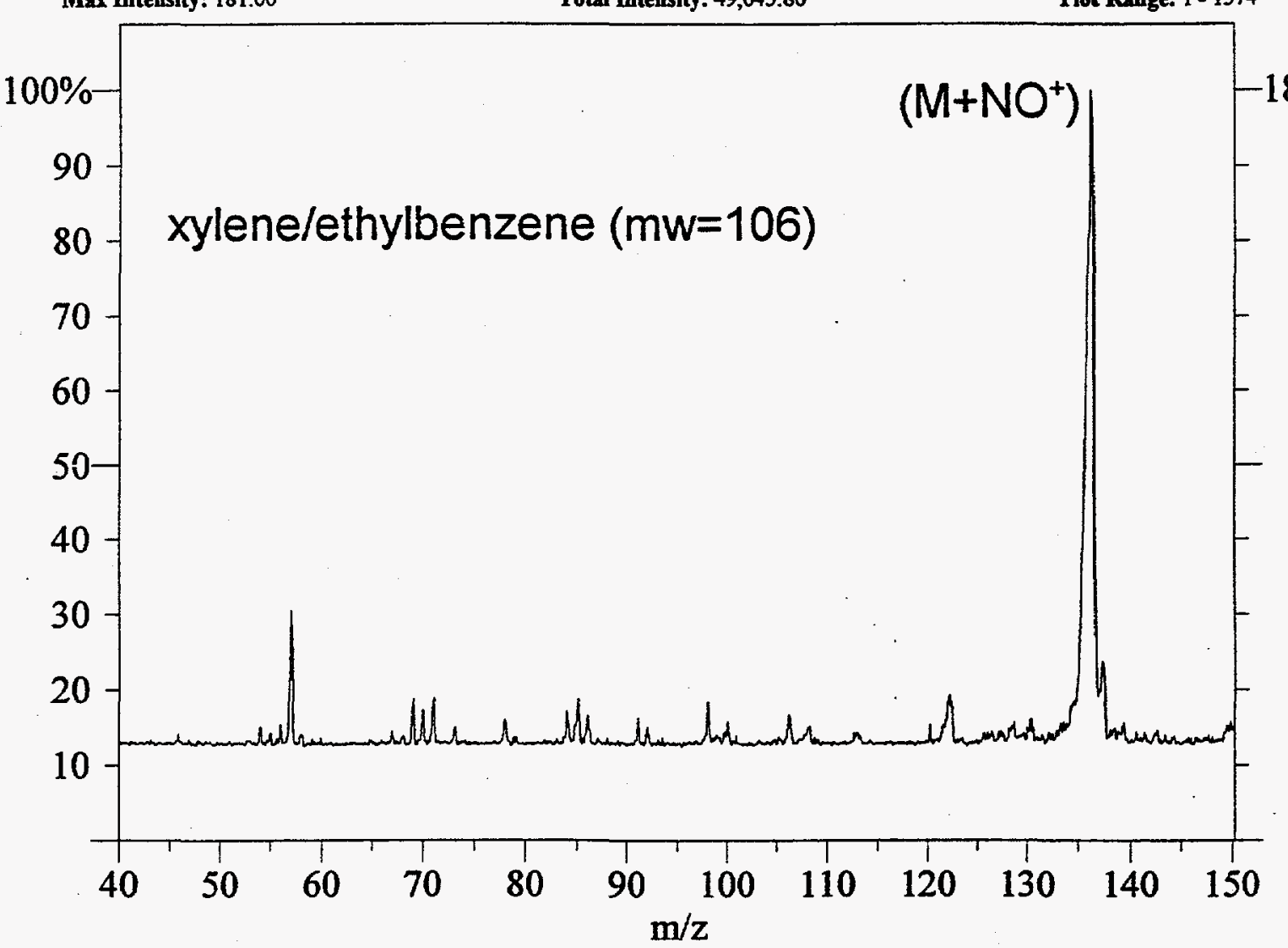

Date: Oct-25-1995

Time: 07:41:00 am

Points: 1998

Points: 1998 Range: 1 - 1374

181
Profile Spectrum Plot

Data File: C:ITELEDYNEL3DQDATA1OCT25D.MS

Sample: ASGDI-pilsed;C2benzenes+NOD

Scan Range: 70-90

Max Intensity: 183.86

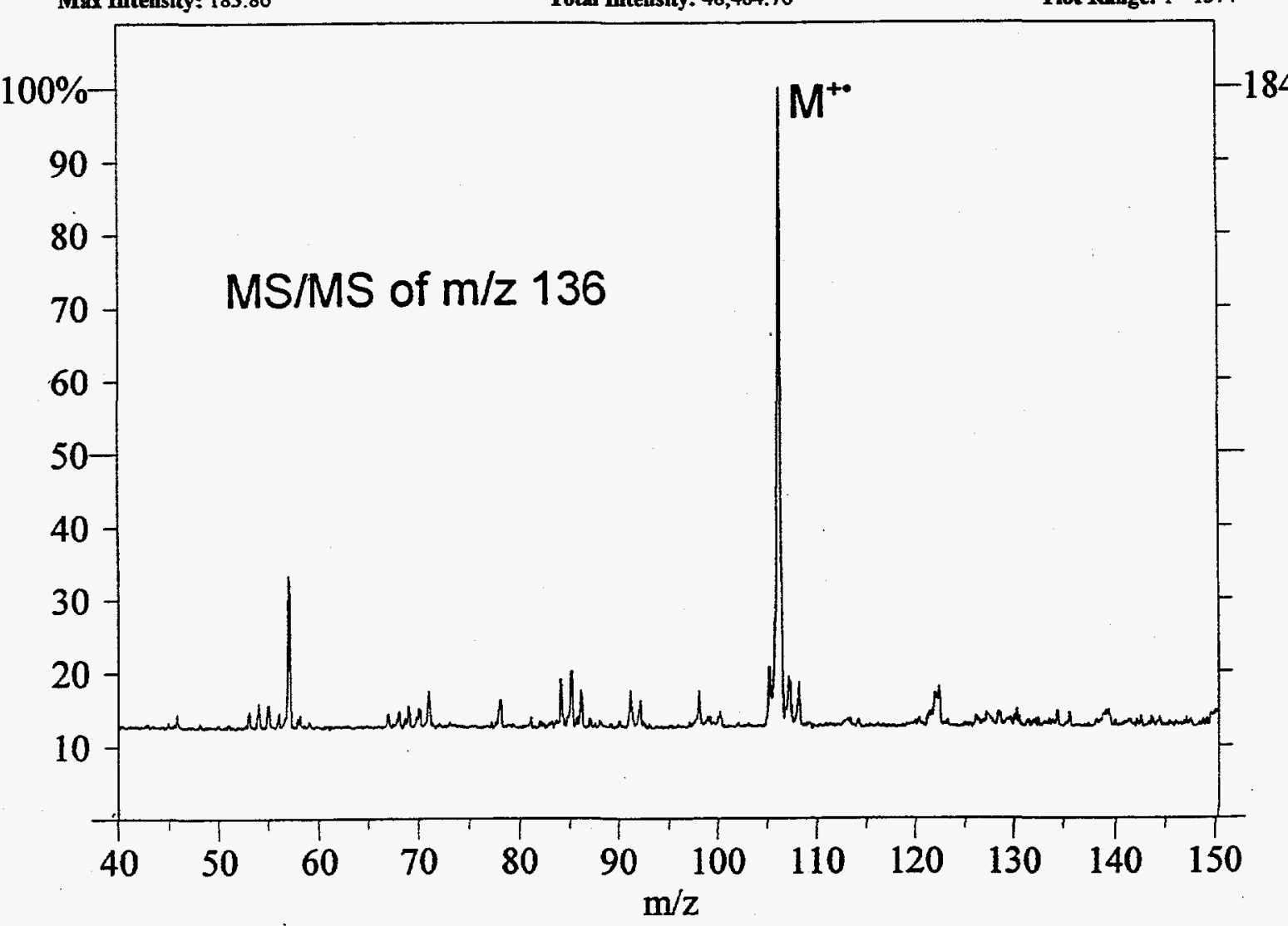

Date: Oct-25-1995

Time: 07:41:00 am

Points: 1998 


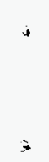

. 


\section{INTERNAL DISTRIBUTION}

1. B. R. Appleton

2. K. G. Asano

3-4. M. V. Buchanan

5. L. B. Dunlap

6. C. A. Valentine, Office of Technology Transfer, 701SCA, M/S-8242.

7. D. E. Goeringer

8. P. L. Gorman, Department of Energy, ORNL Site Office

9. S. D. Hamel, Department of Energy, Office of Patent Counsel

10. K. J. Hart

11-15. S. A. McLuckey

16. A. J. Luffman, CRADA Manager, ORNL-STP Office

17. M. L. Poutsma

18. T. M. Rosseel

19. ORNL-Lab Records

20-21. Lab Records-OSTI

\section{EXTERNAL DISTRIBUTION}

22-23. Mark A. Dearth, Environmental Research Consortium, Ford Motor Company, Dearborn, MI 48121

24. M. Polansky, Director, Advanced Energy Projects and Technology Research Division, Office of Computational and Technology Research, ER-32, Room E-220, Department of Energy, 19901 Germantown Road, Germantown, MD 20874-1290.

25. T. Vojnovich, Laboratory Technology Applications Division, ER-32, Department of Energy, 19901 Germantown Road, Germantown, MD 20874-1290 